\title{
Using Discrete EVent System Specification in Real-Time (DEVSRT) in order to model and simulate artificial pancreas.
}

by

Cheryl Anne D'Souza, B.Eng.

A thesis submitted to the Faculty of Graduate Studies and Research in partial fulfilment of the requirements for the degree of

\author{
Master of Applied Science \\ in
}

\section{Biomedical Engineering}

Ottawa-Carleton Institute for Biomedical Engineering (OCIBME)

Department of Systems and Computer Engineering

Carleton University

Ottawa, Ontario, Canada

December 2015

Copyright (C2015 - Cheryl Anne D’Souza, B.Eng. 
The undersigned recommend to

the Faculty of Graduate Studies and Research

acceptance of the thesis

\title{
Using Discrete EVent System Specification in Real-Time (DEVSRT) in order to model and simulate artificial pancreas.
}

\author{
Submitted by \\ Cheryl Anne D'Souza, B.Eng. \\ in partial fulfilment of the requirements for the degree of \\ Master of Applied Science
}

Supervisor, Dr. Gabriel A. Wainer

Chair, Dr. Yvan Labiche, Department of Systems and Computer Engineering

Carleton University

2015 


\section{Abstract}

One of the key areas that are being paid special attention to is known as red biotechnology (i.e. biotechnology used in the medical field). An example of this is "The Artificial Pancreas' specifically for diabetes mellitus. The device would be composed of a continuous glucose sensor that senses the user's sugar level, an insulin infusion pump that secretes insulin into the user's body when required; and an algorithm that regulates the insulin based on the glucose levels obtained from the sensor. In the past recent years, there have been significant developments in technology that are very specific to the artificial pancreas. Although these devices show promise of future device developments, it currently faces a lot of error and criticism. In order to minimize cost and time to build and verify such a complex system, one must first model and simulate the system. This methodology in turn provides risk-free testing environments and can also be tested with different scenarios.

This thesis will focus on modelling and simulating the artificial pancreas using Discrete EVent System Specification in Real-Time (DEVSRT).This is accomplished by simulating both a meal model and a control algorithm to control the insulin pump based of the data provided by the continuous glucose monitor. The meal model simulation will validate the control algorithm model. The results indicate that the meal model was successfully modelled and validated via comparison to literature. While the control algorithm implemented is not effective when large meals are ingested. 
To my mother, Colette D'Souza, who is my rock and source of encouragement. If I have accomplished anything today it is because of you. 


\section{Acknowledgments}

There are a number of people without whose help this thesis might have not been written and to whom I am greatly indebted.

First and foremost, I thank my thesis supervisor, Dr. Gabriel Wainer, for his support; guidance; and, encouragement throughout these past couple of years. It has been both a privilege and pleasure to work under your supervision.

I would also like to thank the team at AutoDesk, especially Azam Khan, Rhys Goldstein and Simon Breslav, whose support has enabled me to reach my ultimate goal. I would also like to express my appreciation to Mohammad Moallemi, Misagh Ta-

vanpour, Darren Russ, Khi KongChiv and the technical staff of the Department of Systems and Computer Engineering for all the technical help and support.

I owe my deepest gratitude to my family. I attribute my success to their endless love, support and patience. My parents, Colette and William, who have been a constant source of encouragement and inspiration in my life. My brothers, Daryl and Nigel, for always actively supporting me in all my endeavours. 


\section{Table of Contents}

Abstract $\quad$ iii

Acknowledgments $\quad$ v

Table of Contents $\quad$ vi

List of Tables $\quad$ ix

List of Figures $\quad$ X

List of Acronyms xii

1 Introduction 1

1.1 Motivation ........................... 2

1.2 Thesis Goals . . . . . . . . . . . . . . . . . . . 4

1.3 Thesis Organization . . . . . . . . . . . . . . . . 5

2 Review of the State of the Art 6

2.1 Simulation . . . . . . . . . . . . . . . . 6

2.1.1 Simulation of Biological Systems . . . . . . . . . . . 7

2.1.2 Discrete EVent System Specification (DEVS) . . . . . . . . . . 9

2.1.3 DEVS-Based Simulation of Biological Systems . . . . . . . . . 14

2.1.4 Biological Simulation Algorithm Integration . . . . . . . . . . 17

2.1.5 Dynamic Simulation of Chemicals . . . . . . . . . . . . . 17 
2.1.6 Dynamic Simulation of Cells . . . . . . . . . . . . . . . . 18

2.1.7 Dynamic Simulation of Organs . . . . . . . . . . . . 20

2.2 Diabetes Mellitus . . . . . . . . . . . . . . . . . 20

2.3 Literature Overview . . . . . . . . . . . . . . . . . . . . 24

2.3.1 Metabolic models . . . . . . . . . . . . . . . . . . 24

2.3.1.1 Empirical Models . . . . . . . . . . . . 27

2.3.1.2 Semi-Empirical Models . . . . . . . . . . . 30

2.3.1.3 Physiological Models . . . . . . . . . . . . . . 36

2.3.2 Control Algorithm Devices (CAD) . . . . . . . . . . . 38

2.4 Problem Statement . . . . . . . . . . . . . . . . . . . . 44

3 Model Design 44

3.1 Meal Model Simulation . . . . . . . . . . . . . . . . . 45

3.1.1 Plasma........................... 47

3.1 .2 Liver . . . . . . . . . . . . . . . . . . . . . . 51

3.1.3 Muscle and Adipose tissue . . . . . . . . . . . . . . . . 52

3.1.4 Gastrointestinal Tract . . . . . . . . . . . . . . 53

3.1.5 Beta Cells . . . . . . . . . . . . . . . . . 55

3.2 Control Algorithm . . . . . . . . . . . . . . . . . 60

3.2.1 MPC Model Simulation . . . . . . . . . . . . . . 60

3.2.1.1 The glucose absorption . . . . . . . . . . . 61

3.2.1.2 The insulin absorption . . . . . . . . . . . 61

3.2.1.3 The glucose regulatory system . . . . . . . . . 62

4 Simulation Results and Discussion $\quad 69$

$\begin{array}{lll}5 & \text { Conclusion } & 79\end{array}$

5.1 Future Work . . . . . . . . . . . . . . . . . . 80 
Annex A Diabetes Mellitus $\quad 93$

A.1 Regulation of blood glucose . . . . . . . . . . . . . . 94

A.2 Classification of Diabetes Mellitus . . . . . . . . . . . . . 95

A.2.1 Type 1 Diabetes Mellitus . . . . . . . . . . . . . . . 95

A.2.2 Type 2 Diabetes Mellitus . . . . . . . . . . . . . 96

A.2.3 Gestational Diabetes Mellitus . . . . . . . . . . . . . 96

A.3 Long Term Effects . . . . . . . . . . . . . . . . . . . . . . . . . 97

A.4 Current techniques available to monitor and treat diabetes . . . . . 100

A.4.1 Monitoring glucose levels . . . . . . . . . . . . . . . . . 102

A.4.2 Treatment ........................ 103 


\section{List of Tables}

Table 1 Parameters used for the mixed meal model . . . . . . . . . 56

Table 2 Parameters used for MPC model . . . . . . . . . . . . . 66

Table A1 Significance of the results of blood tests used to diagnose diabetes mellitus [35]. . . . . . . . . . . . . . . . . 101

Table A2 Major insulin types and their effective times [40]. . . . . . . . . 104 


\section{List of Figures}

Figure 1 A closed-loop artificial pancreas model: invasive subcutaneous Continuous Glucose Sensor (i.e. CGM); a Control Algorithm (i.e. CAD); and, an insulin pump (adapted from [4]) . . . . . . 3

Figure 2 Illustration of a hypothetical, hierarchical model a meal system. $\quad 9$

Figure 3 A snapshot of a Cell-DEVS simulation depicting the interaction of vesicles and synapsins within a nerve terminal. The above snapshot shows the formation of clusters of vesicles tied by synapsins. 14

Figure 4 A snapshot of a Cell-DEVS simulation depicting a single bolus of dye that is injected into the artery initialised with uniform velocity to detect the narrowing or blockage of arteries. From the left: Simulation of a control test blockage (0\%), medium blockage (35\%), major blockage (52\%) and late stage blockage (70\%) [22].

Figure 5 A graphical image comparing the difference between a normal person's body and one that is afflicted with diabetes (adapted from $[3]) \ldots \ldots \ldots \ldots \ldots \ldots \ldots \ldots$

Figure 6 Block Diagram showing the Artifical Pancreas and Physiological system (adapted from [102]). For more details refer to related image depicted in Figure 7. . . . . . . . . . . . . .

Figure 7 Block Diagram showing the details of MPC (adapted from [102]).

For more details refer to related image depicted in Figure 6. . . 47

Figure 8 Coupled model representing the Liver. . . . . . . . . . . . 51 
Figure 9 Simulation of plasma glucose level of a non-diabetic patient weighing $78.0 \mathrm{~kg}$ graphed in excel . . . . . . . . . . . . . 72

Figure 10 Simulation of plasma insulin level of a non-diabetic patient weighing $78.0 \mathrm{~kg}$ graphed in excel . . . . . . . . . . . . . . . 73

Figure 11 Simulation of Endogenous Glucose Production level of a nondiabetic patient weighing $78.0 \mathrm{~kg}$ graphed in excel . . . . . . . 74

Figure 12 Simulation of Endogenous Glucose Rate Of Appearance of a nondiabetic patient weighing $78.0 \mathrm{~kg}$ graphed in excel . . . . . . . 75

Figure 13 Simulation of insulin dependent Glucose Utilization of a nondiabetic patient weighing $78.0 \mathrm{~kg}$ graphed in excel . . . . . . . 76

Figure 14 Simulation of Insulin secreted by the liver of a non-diabetic patient weighing $78.0 \mathrm{~kg}$ graphed in excel . . . . . . . . . . . . 77

Figure 15 Simulation results of MPC based on a linearised model of the Hovorka model. . . . . . . . . . . . . . . 78 


\section{List of Acronyms}

\begin{tabular}{|c|c|}
\hline Acronym & Definition \\
\hline $\mathrm{ACF}$ & Auto-Correlation Function \\
\hline $\mathrm{AD}$ & Alzheimer's Disease \\
\hline $\mathrm{AP}$ & Artificial Pancreas \\
\hline $\mathrm{APP}$ & Artificial Pancreas Project \\
\hline ARX & AutoRegressive Exogenous \\
\hline AT & Adipose Tissue \\
\hline ATP & Adenosine triphosphate \\
\hline BJ & Box-Jenkins \\
\hline CAD & Control Algorithm Device \\
\hline CDS & Cellular Dynamic Simulator \\
\hline Cell-DEVS & Cellular Discrete EVent System Specifications \\
\hline CFD & Computational Fluid Dynamics \\
\hline CGM & Continuous Glucose Measurements \\
\hline
\end{tabular}




\begin{tabular}{|c|c|}
\hline Acronym & Definition \\
\hline $\mathrm{CHO}$ & Carbohydrate \\
\hline CSII & Continuous Subcutaneous Insulin Infusion \\
\hline DCCT & Diabetes Control and Complications Trial \\
\hline DDE & Delayed Differential Equation \\
\hline DEVS & Discrete EVent System Specifications \\
\hline DEVSRT & Discrete EVent System Specifications in Real-Time \\
\hline DM & Diabetes Mellitus \\
\hline DYNDEVS & Dynamic Discrete EVent System Specifications \\
\hline EGP & Endogenous Glucose Production \\
\hline EIC & External Input Coupling \\
\hline ePID & External Physiological Insulin Delivery system \\
\hline $\mathrm{EOC}$ & External Output Coupling \\
\hline ESRD & End-Stage Renal Disease \\
\hline FDA & Food and Drug Administration \\
\hline FFA & Free Fatty Acid \\
\hline FPG & Fasting Plasma Glucose \\
\hline GDM & Gestational Diabetes Mellitus \\
\hline HLA & Human Leukocyte Antigen \\
\hline
\end{tabular}




\begin{tabular}{|c|c|}
\hline Acronym & Definition \\
\hline $\mathrm{ICU}$ & Intensive Care Unit \\
\hline IDDM & Insulin Depended Diabetes Mellitus \\
\hline IVGTT & Intravenous Glucose Tolerance Test \\
\hline JDRF & Juvenile Diabetes Research Foundation \\
\hline $\mathrm{LVN}$ & Laguerre-Volterra Network \\
\hline$m l$-DEVS & Muti-Level Discrete EVent System Specifications \\
\hline MNT & Medical Nutrition Therapy \\
\hline MPC & Model Predictive Controllers \\
\hline M\&S & Modelling and Simulation \\
\hline NARX & Nonlinear AutoRegressive with eXogenous input \\
\hline NIDDM & Non-Insulin Depended Diabetes Mellitus \\
\hline $\mathrm{NN}$ & Neural Network \\
\hline ODE & Ordinary Differential Equations \\
\hline OGTT & Oral Glucose Tolerance Test \\
\hline$\rho$-DEVS & Port Discrete EVent System Specifications \\
\hline $\mathrm{PD}$ & Proportional Derivative \\
\hline PID & Proportional Integral Derivative \\
\hline PDE & Partial Differential Equations \\
\hline
\end{tabular}




$\begin{array}{ll}\text { Acronym } & \text { Definition } \\ \text { Ra } & \text { Rate of Apperance } \\ \text { RBF } & \text { Radial Basis Function } \\ \text { RT } & \text { Real-Time } \\ \text { SMBL } & \text { Systems Biology Markup Language } \\ \text { T1DM } & \text { Type 1 Diabetes Mellitus } \\ \text { T2DM } & \text { Type 2 Diabetes Mellitus } \\ \text { TPS } & \text { Tethered Particle System } \\ \text { USD } & \text { United States Dollar } \\ \text { WHO } & \text { World Health Organization }\end{array}$




\section{Chapter 1}

\section{Introduction}

A control system is a device, or set of devices to manage, command, direct or regulate the behaviour of other device(s) or system(s). By this definition one can easily see how many, if not all, biological systems can be thought of as control systems. However, even though this link has been established for a number of years (dating back to 1865 [1]), it was only in the past recent years that there has been any significant impact in the biological realm.

Biotechnology can be further broken down into four different fields - red, blue, white and green biotechnology. Red biotechnology, which this thesis will focus on, is biotechnology that is used in the medical field, often referred to as biomedical. Blue biotechnology refers to application in aquatics. Whereas white biotechnology refers to industrial application and green biotechnology refers to agriculture and plant usage.

There are two main reasons why red biotechnology is quite different from other areas that utilise control systems:

- It has a greater and more direct impact to human life than any other field; and,

- It is a far less mature and less established field than any of the other fields such chemical, aerospace, electrical, etc. 
Despite these barriers, there has been significant developments in the recent years in the areas of cardiovascular and endocrinology. More specifically it is the development of the artificial pancreas that this thesis focuses on.

Regardless of the application, in order for something to be developed and used especially where human life is concerned, it has to be thoroughly tested and verified. An effective, reliable and cost effective way to develop and test designs of these devices is via Modelling and Simulation (M\&S). More specifically computer-based M\&S proves to be an effective tool due to its efficient analysis, design, optimisation and verification of systems. Furthermore, the use of M\&S in software engineering reduces costs, risks and allows different aspects of the system to be closely examined if necessary.

Formal M\&S is a specific branch of M\&S that defines models using formal specification tools. There are a number of different formal specifications that are used for various systems. With respect to biomedical devices the most common and commercial specifications include but are not limited to SMBL, Matlab, Mathematica, and Modelica. However, for this thesis a more unconventional modelling formalism was used - Discrete EVent System Specification. DEVS was chosen over the other modelling techniques mainly because it has been time and again proven to be successful and easily used when modelling complex applications. This is mainly due its ability to reuse models, fast prototyping and incremental development of models [2].

\subsection{Motivation}

In the year 2012, it was estimated that approximately 382 million of the adult world population was affected with diabetes mellitus. In addition to this, there are ap-

proximately 316 million with impaired glucose tolerance and are at high risk of being diagnosed with diabetes mellitus. This number is said to grow to a estimated 471 
million by the year 2035. With these alarming numbers, it is not surprising that many consider this disease to be a global epidemic of the 21st century. Like most epidemics, the burden does not lie solely on those afflicted with the disease. It also affects the community as a whole due to the high premature fatality rate and sky rocketing health care costs, incurred both directly (USD 263 billion) and indirectly (i.e. paid sick leave, restricted activity days, lower productivity at work, premature mortality and permanent disability which amount to $50 \%$ of the direct costs associated with the disease) [3].

With the current treatment options available; these epidemic like numbers are not expected to decline anytime in the near future. Thus, the only solution to this is to develop something that would result in a more effective treatment of the disease. Since the disease is caused by a faulty organ in the human body, the trivial solution would be to replace it with something that mimics its functionality - an artificial pancreas - a portable closed loop insulin delivery system.

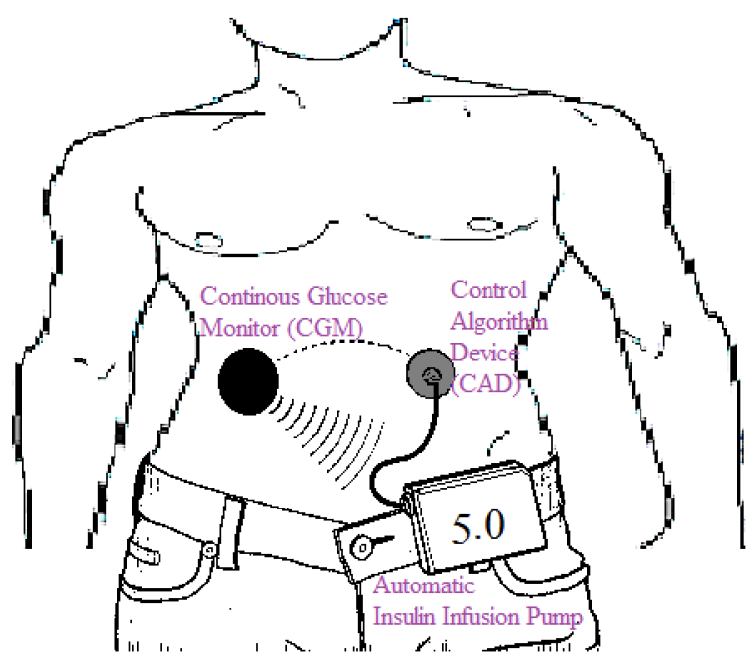

Figure 1: A closed-loop artificial pancreas model: invasive subcutaneous Continuous Glucose Sensor (i.e. CGM); a Control Algorithm (i.e. CAD); and, an insulin pump (adapted from [4]).

The basics of this closed loop strategy is quite simple and involves three main aspects: 
a CGM device; a Control Algorithm Device (CAD); and, an insulin pump (see Figure 1) [4]. The CGM is an invasive subcutaneous device that is used to continuously measure the glucose concentrations in a patient's cells. The CAD then receives the readings from the CGM and uses a predefined algorithm to calculate the concentration of insulin that needs to be pumped into the patient's body. It then sends the calculated value to the insulin pump. The insulin pump automatically dispenses the correct dosage of insulin as instructed by the CAD.

In 2006, the Juvenile Diabetes Research Foundation (JDRF) [5] recognised the need for the above mentioned closed loop strategy to be implemented. This provided a way to what is now known as the Artificial Pancreas Project (APP) Research. The launch of this project solved the first problem - i.e. the availability of a subcutaneous glucose sensing device. This device was key because it was not only invasive but it needed to sense and actuate intravenously such that it minimises discomfort to the patient. However, this resulted in a second problem - an added delay within the closed loop circuit that would result in more challenges when considering the development of the control algorithm.

\subsection{Thesis Goals}

As described in section 1.1, currently there isn't a single system that can gracefully act as an artificial pancreas. There are major building blocks that do exist; but, the integral part - the control algorithm device does not yet exist in its entirety. The goal of this thesis is to tackle the problem by developing a meal model simulator and a control algorithm in DEVS. This is achieved by first evaluating the various meal model simulators and control algorithms that have currently been developed for completeness and accuracy. Thus in turn establishing a gold standard for both the meal model simulation and the control algorithm. 
The next step would be develop the meal model simulator in a hierarchical format in DEVS and to test it by comparing it to the expected outputs for a given input. It is important to note that a hierarchical structure is crucial to the development of a meal model simulator because one can add or omit certain aspects of the human body as needed. This would then result in validating both complex problems as well as simpler problems. Lastly, this thesis aims to develop a control algorithm that is based in DEVS and test it against the meal model simulator previously built to assess for its effectiveness.

\subsection{Thesis Organization}

The first two chapters provide an introduction to the thesis itself; where, chapter 2 provides insight on previous research conducted including DEVS formalism. In addition, it provides a background on the disorder modelled, Diabetes Mellitus; and, discusses the various models that describe the relationship between glucose and insulin in the human body. It concludes by stating the problem statement of this thesis.

The next chapter discusses how the problem posed in the introductory chapters was tackled. This is accomplished by describing how the model chosen in chapter 2 was implemented into DEVS, followed by description of the simulation results.

The next section of the thesis discusses the project itself and possible future work; including how the work presented in this thesis can be extrapolated to model some other organs using the DEVS formalism. This is done in two chapters where chapter 4 presents the discussion and chapter 5 presents the conclusion and insight into possible future work.

The thesis concludes with an annex that provides in depth insights into the disease itself. 


\section{Chapter 2}

\section{Review of the State of the Art}

As the title suggests, this chapter reviews the state of the art. It is broken into four different sections. The first serves as an introduction to the simulation side of the thesis. The second serves as an introduction to the disease Diabetes Mellitus. This is followed by a literature overview of the available control algorithms. The chapter concludes by defining the problem statement.

\subsection{Simulation}

This first section which serves as an introduction to the simulation side of the thesis is outlined as follows: Sub-section 2.1.1 provides an introduction to the realm of biological systems from the perspective of simulation. Following which, the system specification used to simulate the biological system of interest will be briefly discussed. The section then moves onto discussing how the two above mentioned sub-sections connect together (sub-section 2.1.1 \& sub-section 2.1.2). Lastly, the section concludes by giving a brief overview of how the algorithms are integrated in previous research. 


\subsubsection{Simulation of Biological Systems}

Many times, the words model and simulation are interchanged in everyday conversation. However, in the M\&S community the term 'model' is referred to the particular aspect of the real-world system that is closely examined. The word 'simulation', is used to describe the process used to imitate the system through a particular model. Additionally, the term 'simulator' refers to a program that is designed for the sole purpose of running simulations. That being said, it should be noted that a model can be described by a number of methods; some of which are informally based and some are formal descriptions widely used. The formal descriptions generally involve a number of techniques including but are not limited to: equation, diagrams and prose [6]. However, when referring to formal description, this document will for the most part only examine mathematical formulas. Similarly, when this document refers to simulating a system, it only includes the design of the formal model and the use of a technology to calculate the outcomes of certain situations based on the formulas described in the model.

Simulating biological systems poses many technical challenges that are often not present in other real systems. This is mainly due to the fact that biological systems are governed by the laws of physics as well as chemistry whose interactions by itself are not well understood and documented [7]. This difficulty mainly has two reasons behind it. First, even the most basic system in the biological realm is very complex to understand due to its many inter-dependencies on other systems. Thus, when medical researchers try to model this complex system it tends to become even more complex due to several limitations including the modelling framework itself. Secondly, in order to simulate something realistically, most biological systems require many complex algorithms to be integrated together failing which, one or more key behaviours of the system would be undefined. For example, with respect to modelling a pancreas, not 
only does one have to develop and implement an algorithm for blood sugar regulation, but also for muscle and adipose tissue absorption, enzymes from the liver, etc. This in turn increases the scope of the project immensely.

However, in [8] the authors note that many of these complex large systems can be broken down into smaller subsystems by conceptually modularising the entire system in an analogous manner. Many real systems today have been modelled and simulated by adopting this approach. The Discrete EVent System Specification (DEVS) is one such formalism that allows one to break a model into smaller components with ease $[6,9]$. This is mainly done by the brilliance of the DEVS simulation program itself. It allows the user to model any into simpler sub-models. These sub-models can then be further divided into sub-models of its own. This can be done repeatedly as many times as the user wants to create a complex yet fully defined hierarchical structure. There is a small downside to this methodology and that is the fact that extra code must be written to define the hierarchical structure. But, this is a small cost to pay considering that each sub-model must be designed independently and can be reused at any time to aid in other simulations.

An example of a hierarchical model is depicted below in Figure 2. The upper most level of the hierarchical model represents the entire meal system with respect to it's interactions with the entire human body that is relevant to the production of insulin. The nodes in the lower level represent the different components involved. The arrows indicate the direction in which the signals are sent from one component to the next. It should be noted that the figure is just an illustration and by no means represents an entire meal system. 


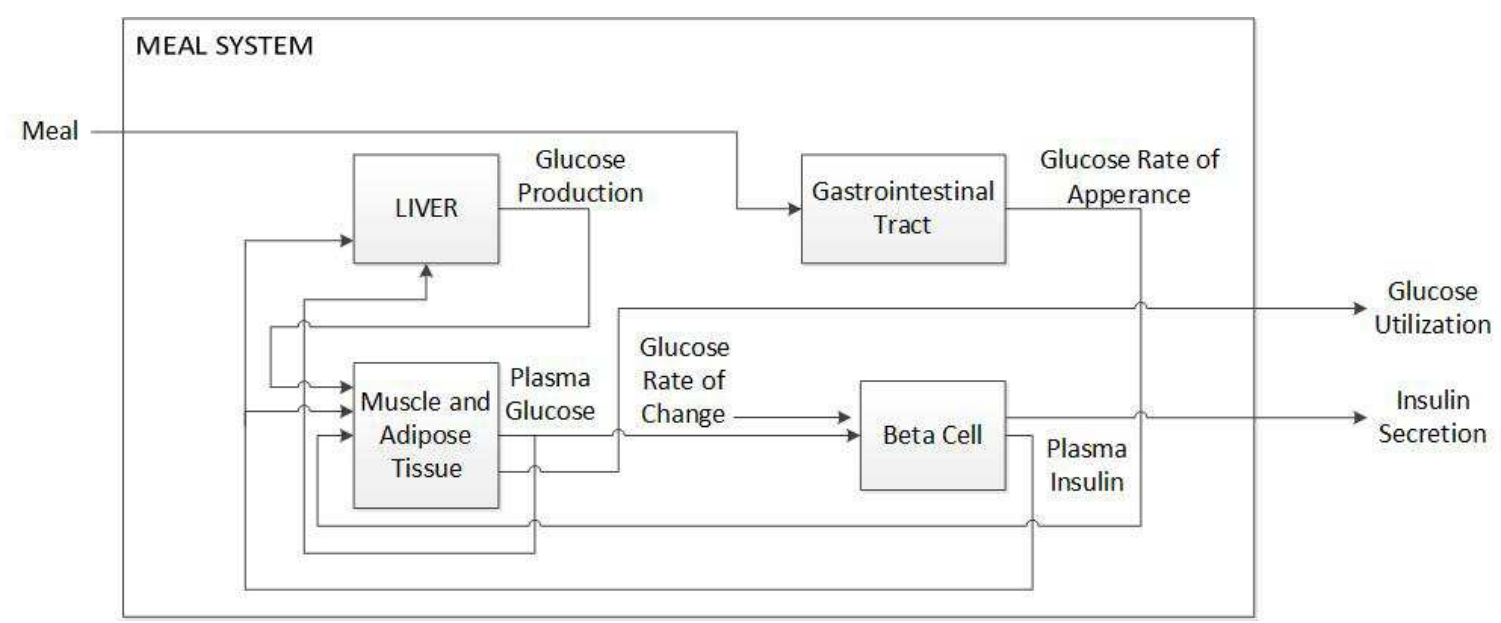

Figure 2: Illustration of a hypothetical, hierarchical model a meal system.

\subsubsection{Discrete EVent System Specification (DEVS)}

In 1976, Bernard Zeigler invented the Discrete EVent System Specification (DEVS) mainly because he was convinced there was a need for a novel theory in the realm of discrete event simulation. DEVS is a general formalism that defines a number of conventions to formally describe a wide range of systems that vary with time [10]. There are many key distinguishing factors that DEVS formalises. These include but are not limited to the distinction between system and constant states, the design of hierarchical models and the separation between simulators and models. Ever since its first proposal and implementation, DEVS has been used as a basis for many other variant formalisms that are widely used today.

It should be noted that while many of the real world systems change continuously with time, the states in a discrete event simulation changes only at discrete times. However, DEVS compensates for this disadvantage by introducing a 'system state' - a state that represents the real world system and distinct from the 'constant state' that is handled by the computer. The system state is comprised of two main components: the constant state and the time elapsed since the previous event. Unlike the constant state, the system state is continuously changing because the elapsed time is not 
constant. The DEVS transition function then calculates the new constant states based on the current system state [11]. For the purposes of this document, the term 'constant state' will henceforth be referred to as 'state'.

In section 2.1.1 it was stated that simply put, a model is a description of a system. More specifically, a DEVS model is a formalised description of a system that is driven with a set of mathematical functions. It was also stated that DEVS uses a hierarchical structure to build a model. The smallest component of this structure is called an 'atomic model' and is defined:

$$
<X, Y, S, \delta_{\text {ext }}, \delta_{\text {int }}, \lambda, t a>
$$

Where:

$X$ refers to the input values of the DEVS atomic model;

$Y$ refers to the output values of the DEVS atomic model;

$S$ refers to the states associated by the DEVS atomic model;

$\delta_{\text {ext }}$ refers to the external transition function;

$\delta_{i n t}$ refers to the internal transition function;

$\lambda$ refers to the output function; and,

ta refers to the time advance function.

One of the advantages of using DEVS is that although formally one has to define $\delta_{\text {ext }}, \delta_{\text {int }}, \lambda$; and, ta; they do not need to need to worry about explicitly calling them. This is because the fore mentioned functions are handled by the simulator itself (Note: the simulator and the model itself are separate). The first three parameters on the other hand need to be defined and explicitly called by the model. This leads into another advantage of DEVS, which is, that the simulator itself is not model specific. Thus, any well-defined DEVS model, regardless of what the model represents can be simulated with ease [2]. 
The way a simulator executes an atomic model is as follows: The simulator initially has the DEVS model in its initial state (defined by the parameter $S$ ). After any event, the simulator then evaluates the time advance function (defined by $t a$ ) in order to determine when the next internal transition should occur. Once this time has elapsed, the output function (defined as $\lambda$ ) is executed and the output values (defined as $Y$ ) are determined. Upon completion of these events, the model is now in a new state. However, before the time advance function elapses, if the simulator is interrupted with an input value (defined as $X$ ), the simulator applies the external function (defined as $\left.\delta_{\text {ext }}\right)$ to transition into it's new state. Thus, the internal transition function, $\delta_{i n t}$, has only one input argument - the state, $S$ and thus effectively is a function of the system state for a guaranteed elapsed time of $t a(s)$. The external transition function, $\delta_{\text {ext }}$, has three input arguments - the current state, $S$; the elapsed time, $\Delta t_{\text {elapsed }}$; and, the input value $X$. The external transition function is by definition a function of the system state because it contains both arguments $-s$ and $\Delta t_{\text {elapsed }}[2]$.

The other type of model that DEVS uses is called a 'coupled model'. These models are comprised of a number of sub-models which themselves can be atomic or coupled models. Note that due to DEVS' hierarchical model it allows coupled models to be nested in each other. A coupled model structure is defined as:

$$
<X, Y, D,\left\{M_{D} \mid d \in D\right\}, E I C, E O C, I C, \text { Select }>
$$

Where:

$X$ refers to the input values of the DEVS coupled model;

$Y$ refers to the output values of the DEVS coupled model;

$D$ refers to the model identifiers of the DEVS model;

$\left\{M_{D} \mid d \in D\right\}$ refers to the model identifiers that are specific to this DEVS coupled model; 
$E I C$ refers to the external input coupling;

$E O C$ refers to the external output coupling;

$I C$ refers to the internal coupling; and,

Select refers to the function that determines which sub-model needs to undergo an internal transition in the event of a tie.

The best way to explain how a coupled model works is through example. Suppose the meal system model depicted in Figure 2 is considered as a coupled model. The four atomic models are Liver; Muscle and Adipose Tissue; Gastrointestinal Tract; and, Beta cell. X would be defined as the input values, i.e. the meal ingested itself; and Y would be the output values, i.e. The Glucose utilisation by the body and the Insulin Secretion. The variable D would be defined by the set of model identifiers, i.e. the atomic model involved (in this case Liver; Muscle and Adipose Tissue; Gastrointestinal Tract; and, Beta cell). The tuples of the four atomic models would be included in $\left\{M_{D} \mid d \in D\right\}$. The external input coupling set EIC is represented by the arrow leading into the meal system itself (i.e. the meal to the Gastrointestinal Tract). Similarly, the external output coupling set EOC is represented by the arrows leading out of the meal system (i.e. the arrow from the Muscle ad Adipose tissue to Glucose utilisation; and, from the Beta Cell to the Insulin Secretion). Messages between the atomic models are passed through the internal coupling set IC, which is represented by the arrows between the four atomic models (i.e Glucose Production; Plasma Glucose; Glucose Rate of Appearance; and, Plasma Insulin). Last but not the least, a Select function is defined in order to determine which atomic model undergoes an internal transition first in the event of a tie.

Over the years, DEVS has been applied to a number of different types of models. One of such applications is the Cellular Discrete Event Specification (Cell-DEVS) which applies DEVS to cellular models [12]. The advantage of Cell-DEVS is that 
the variables in equations 1 and 2 do not have to be explicitly defined. However, for every Cell-DEVS coupled model the modeller can opt to either define a DEVS coupled model; or, define a cell-space. The communication between cells are based on its own current state and the state of its neighbours. This proves to be a lot simpler than defining messages between systems or cells. Another advantage of Cell-DEVS as described in [12] is updating particular or all the cell states asynchronously as opposed to the traditional way (nested loops that update at a specified regular time interval). Last but not the least the advantage of integration. The modeller can chose to define the entire system in Cell-DEVS or define certain components in Cell-DEVS and the rest in DEVS. Having this flexibility proves to be extremely useful in terms of reusing a part of past models or in its entirety.

Applications of continuous space models in DEVS however are an extremely rare find. An example of such a case would be the forest fire model that is described in [13]. This particular example integrates a variation of DEVS designed in particular for dynamic structures [14]. The model itself describes a forest fire front that is represented by a set of points connected to each other. Each point on this from is described by a particular DEVS atomic model and as the simulation continues, the fire grows (new sets of connected points are added). Like everything, a model like this has its own advantages and disadvantages. The advantage lies in the fact that continuous-space is easily handled by the use of vectors and cellular models that distinguishes one cell from another. The disadvantage of such a system would be the multiple nested boundary system that is needed to accurately describe the temperature variations in the different cells [15]. 


\subsubsection{DEVS-Based Simulation of Biological Systems}

There is a wide spread use of simulation when it comes to studying biological systems. Additionally, DEVS formalism also has gained a lot of popularity along the years. But, the combination of the two is relatively rare. One notable group, led by Dr. Gabriel Wainer at Carleton University, applied Cell-DEVS to cellular biological systems [16]. An example of one such model is the vesicle-synapsin interactions [17]. The model (snapshot depicted in Figure 3) prevents the collisions between arbitrarily large clusters using rules that are restricted to a 5-cell neighbourhood. The results indicate the behaviour of vesicles and synapsin in neurons were captured in a qualitative sense.

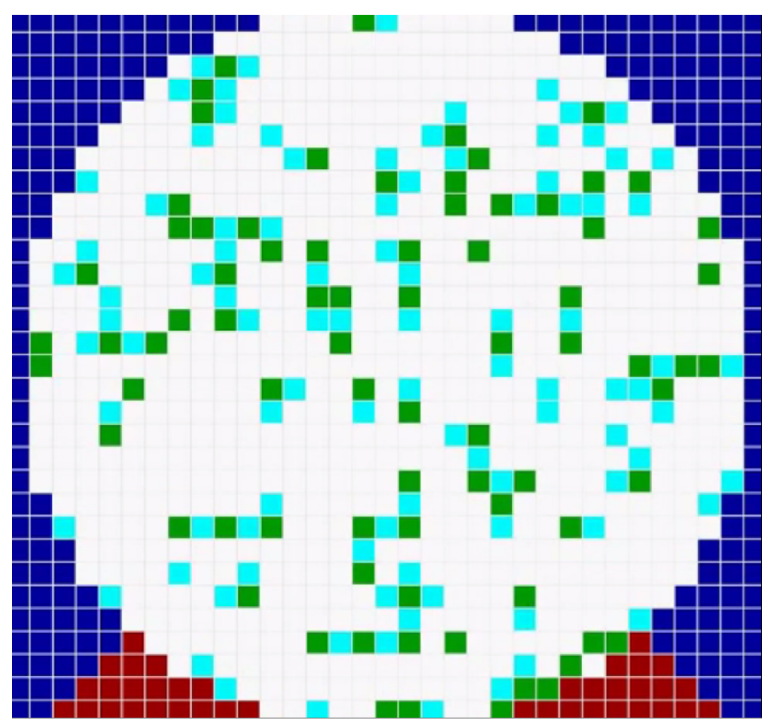

Figure 3: A snapshot of a Cell-DEVS simulation depicting the interaction of vesicles and synapsins within a nerve terminal. The above snapshot shows the formation of clusters of vesicles tied by synapsins.

Dr. Adelinde M. Uhrmacher leads another group at the University of Rostock that applies DEVS to various non-spatial biological models. An example of this can be found in [18] where the coupled model represents an enzyme called the 'RNA Polymerase'. Messages are then passed to the sub-model called 'promoter' which then 
decides if the RNA polymerase can bind to it or not. The non-spatial aspect of this particular model comes into play because of the positions of the RNA polymerase and the various other biological structures are not stored and used.

Theoretically, continuous space biological models could be easily designed in DEVS. One could take the pre-existing models such as the forest fire described in [15] and used vectors to track chemicals, enzymes, etc. that are in the blood stream. However, a literature search resulted in minimal continuous space DEVS models that involve biological systems that can be found in living organisms. The most prominent one is described in [6], where an impulse-based method for simulating dynamics of deformable structures such as a presynaptic nerve terminal model.

When considering simulating a particular model with a given formalism such as DEVS, one must first put forth the question: is it possible to use a formalism at all and if sub models can be chosen effectively to exploit its biggest advantage - hierarchical structure definition. Theoretically, it is quite possible to represent an entire complex biological system in one single atomic model. If this is the case, the modeller might not chose not to describe the model in the DEVS formalism since the only advantage they will gain is the separation between the simulator and the model itself. One the other hand, internal and external transition $\left(\delta_{\text {int }}\right.$ and $\delta_{\text {ext }}$ respectively) functions might potentially contain various looks and data structures that are way more complicated than necessary. Thus, the only way to fully ensure that the true benefit of DEVS is utilised is to use coupled models that are further subdivided into simpler sub models which in turn leads into simpler and smaller internal and external transition functions ( $\delta_{\text {int }}$ and $\delta_{\text {ext }}$ respectively).

Although the above mentioned proposition sounds easy, designing an effective hierarchical structure poses a huge challenge. This is particularly true when examining biological systems that contain many interdependent components. DEVS formalism 
basically has three alternatives when it comes to hierarchical modelling. These are stochastic petri nets; stochastic $\pi$-Calculus; and, state charts [19]. The University of Rostock tackles the problem of designing a hierarchical structure for biological systems by representing biological entities (such as cell components including but not limiting to the cell membrane and nucleas) as coupled models. The interactions between the entities are then handled in accordance to the DEVS formalism. The problem lies in the fact that DEVS passes messages between related models (coupled or atomic) independently of one another. Because of this many people claim that DEVS is unsuitable for the modelling interactions that involve more than two entities [19].

However, one should note that this problem is not unique to DEVS. It manifests itself in every biological model that is bases model hierarchies on biological entities [6]. Although this deficiency is not solved in its entirety, there are many other extensions of DEVS that improve its efficiency. Three such examples include but are not limited to DYNDEVS [9], $\rho$-DEVS [20] and ml-DEVS [21]. DYNDEVS extends the traditional formalism of DEVS by allowing the model's description to change its own state and behaviour pattern as needed. This allows the model transitions to generate a sequence of successive models. The idea behind this is that a system is more likely to change its composition; behavioural pattern; or, it's interaction structure rather than the interface to its environment [9]. $\rho$-DEVS is an extension of DYNDEVS. The difference with this extension however lies in the fact that the interface to its environment is not necessarily static and can change based on its input and output ports (and hence multi-coupling was introduced) [20]. Lastly, ml-DEVS takes it one step further, by introducing coupled models with its own state and behaviour; and, the formalism explicitly defines how the macro-level and micro-level affect each other [21]. As seen in the above descriptions, as DEVS is modified to aid in the simplification of biological 
systems, the formalism itself becomes exponentially complicated and difficult to learn.

\subsubsection{Biological Simulation Algorithm Integration}

One of the best examples of a biological algorithm integration in DEVS is described in [6]. This work applies DEVS formalism to biological models with continuous space by performing a dynamic simulation of deformable biological structures, namely vesicle synapsis in a nerve terminal. This is done by using a method called tethered particle system (TPS) to simulate the deformable structures. The TPS methodology has a special unique way to capture the gradual deformation of a deformable object using instantaneous impulses that occur when two or more particles collide. Additionally, there are many other biological models that simulate the diffusion of chemical, computational fluid dynamics [22], etc.

However, there is a huge desire to integrate biological algorithms that are described within different formalisms. This is due to the belief that it would ease the modelling and simulation process, a modeller must take advantage of the different formalisms that are available. Furthermore, certain sub-components of the model might be already available in the $M \& S$ community and thus having a way to model something using 'multi-formalism modelling' proves to be ideal. This ideology provided a way to the framework James II which combines both the stochastic and spatial variants of the $\pi$-Calculus with different variants of DEVS. This formalism is now called Beta Binders and is described in [23].

\subsubsection{Dynamic Simulation of Chemicals}

The phrase 'dynamic simulation' is often used to describe a simulation of something in motion using the laws of classic dynamics. This section in particular reviews the various applications that simulate chemicals using classic dynamic principles. There 
are many models that simulate chemicals and how they react within a certain system.

An application of this can be seen in [24] which presents a Cellular Dynamic Simulator (CDS) for simulating the diffusion and chemical reactions within a crowded molecular environment. The interesting thing that this work presents is that the simulator is event-driven and is based on a common language Systems Biology Markup Language (SMBL). However, it is constrained by the space in which the simulation can take place. The human body, though definite in space, has a large number of chemicals which are used though out the body and is best represented by a continuous-space model.

\subsubsection{Dynamic Simulation of Cells}

The human body is made up of cells, tissues and organs. According to [25], cells are defined as the basic structural building block of a living organism. Tissues are made up of unique specialised cells. For example only a nerve cell can make up nerve tissue (also known as ganglia). In essence one can think of tissues as a large group of cells, that perform the same function in the human body. Organs are made up of a group of tissues that work together to perform one or more specific tasks. For example eyes are considered an organ in the human body because it is made up of nerve tissue, lens tissue, blood vessel tissue, muscle tissue, etc. All these various types of tissues work together to allow a person to see.

There are many applications of dynamic simulation of cells around the world. One noted application makes use of Computational Fluid Dynamics (CFD). Traditionally, CFDs were solved using various numerical methods that would attempt to provide an accurate approximation [26]. However, recently a novel approach using DEVS formalism was used to analytically solve a CFD problem. The approach was used to 
simulate the effects of coronary artery disease. It does this by simulating a single bolus of dye that doctors usually inject into a patient to detect any narrowing or blockages in the artery. The simulation used the Navier-Stokes equations to approximate the flow of blood in arteries. Figure 4 presents different scenarios of coronary artery disease blockage such as a control test blockage (0\%), medium blockage (35\%), major blockage (52\%) and late stage blockage (70\%) [22].
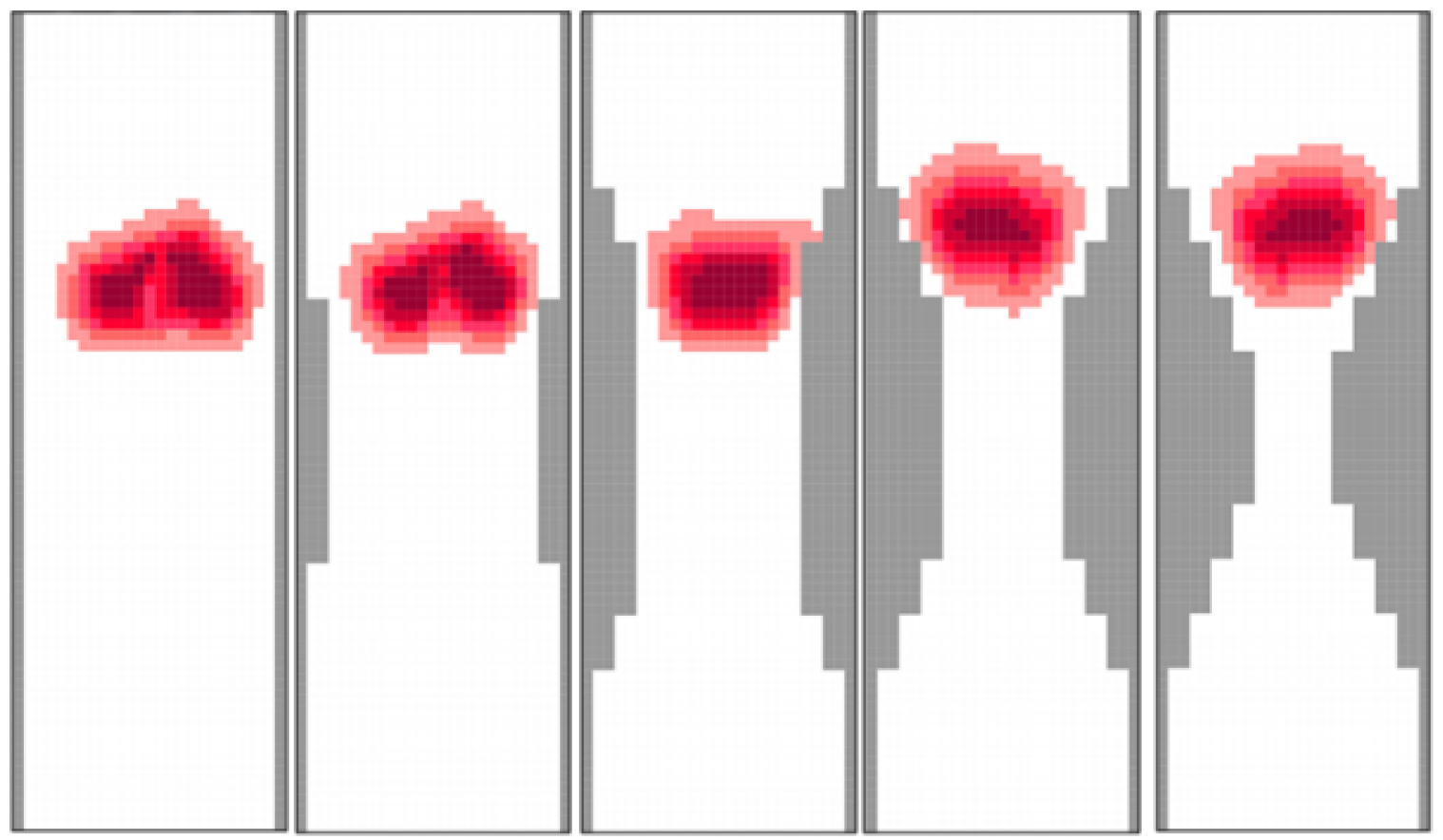

Figure 4: A snapshot of a Cell-DEVS simulation depicting a single bolus of dye that is injected into the artery initialised with uniform velocity to detect the narrowing or blockage of arteries. From the left: Simulation of a control test blockage $(0 \%)$, medium blockage $(35 \%)$, major blockage $(52 \%)$ and late stage blockage $(70 \%)[22]$.

However, the above mentioned model is a cellular model which has a tendency to restrict motion and thus has a negative impact on the simulation as a whole. Thus, for biological systems, a continuous-space system preferred. 


\subsubsection{Dynamic Simulation of Organs}

As explained in section 2.1.6, an organ is a group of tissues that work together to perform one or more functions. Due to its complexity, there are a limited number of models that exist that are modelled in continuous-space and using the laws of classical dynamics. One such example is documented in [6]; which simulated a pre-synaptic nerve terminal. The key to the dynamic simulation outlined in this work is the use of the Tethered Particle System (TPS) method which makes use of the particle collision theory. The impulses generated by each particle collision is then used to dynamically simulate impulse of deformable structures. However, this work focuses on simulating biological systems at a low level and is more applicable to simulating chemicals and particles as opposed to entire organ.

\subsection{Diabetes Mellitus}

Diabetes Mellitus (DM) has plagued the world to a point where it imposes high social, economic and human costs around the world. The international diabetes federation estimates that 382 million people around the world are affected with diabetes and this number is estimated to rise to approximately 592 million in less than 25 years. They also expect 175 million people are afflicted with the disease and are either under diagnosed or not diagnosed at all. As it stands, these alarming numbers only suggest that the world is losing the battle to conquer the disease which in turn can be viewed as a major threat to global development. This section aims to provide only a brief overview on the definition of the disease; classification of the common types of diabetes; and, the current techniques available to treat and monitor the disease. Note: Refer to Annex A for additional information on the disease.

To begin one must first understand what exactly the disease entails. A person is said 
to be diagnosed with DM when their body (specifically the pancreas) cannot produce the hormone insulin, or, their body cannot use insulin effectively. Insulin, a hormone produced in the pancreas of the human body, is what triggers all the cells in the human body to absorb glucose from the food ingested. The cells are then responsible for transforming the glucose into energy (ATP) which is then utilised by various tissues and muscles to perform their day to day tasks. A person who is afflicted with this disease cannot absorb glucose properly for various reasons. This means that the glucose absorbed by the body circulates the blood stream and damages the body's tissues and muscles over time. These damages can result in enormous life threatening complications. There are three major types of diabetes (see Figure 5):

- Type 1 Diabetes Mellitus (T1DM)

- Type 2 Diabetes Mellitus (T2DM); and,

- Gestational Diabetes Mellitus (GDM)

T1DM is an autoimmune disease which often times occurs suddenly. It is occurs in humans where the pancreas (an organ responsible for the production of insulin) no longer produces the hormone because the body's defence system attacks it $\beta$-cells (cells located in the pancreas which produces insulin). This in turn does not permit glucose from entering into the cells which then cannot produce energy for it's survival. In order to prevent hyperglycemia (a condition when high concentrations of glucose in the blood stream), the person must inject himself/herself with the insulin hormone. Failure of which will lead to the slow deterioration of organs which in turn then leads to death. On the bright side, studies have shown that people afflicted with DM can survive if their glucose levels are closely monitored.

The pancreas of patients afflicted with T2DM and GDM on the other hand produce insulin. However, the cells in the body are resistant the insulin hormone; or, the 


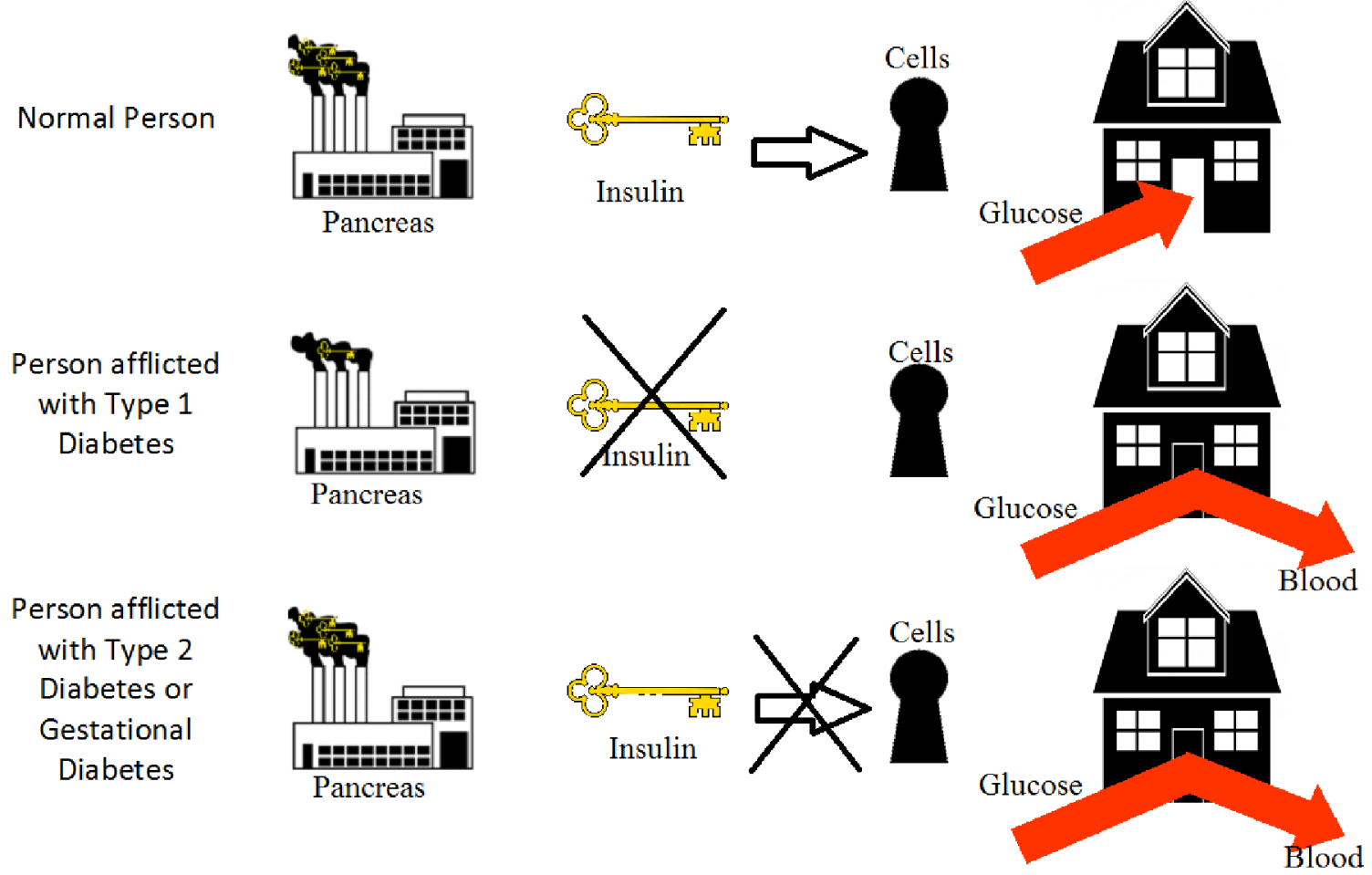

Figure 5: A graphical image comparing the difference between a normal person's body and one that is afflicted with diabetes (adapted from [3]).

$\beta$-cells located in the pancreas do not produce enough insulin for the body's needs. Rejection of insulin or inadequate amounts of insulin results in avoiding or suppressing the cell's intake of glucose. Many people who are afflicted with T2DM are undiagnosed and thus are more prone to suffer the long term damages of the disease. GDM, as it's name suggests, occurs only in women, and appears during pregnancy. This can lead to serious health risks for both the mother and in infant. Although this form of diabetes usually vanishes upon giving birth to the baby, it greatly increases the chances of developing T2DM. Unlike T1DM, patients with T2DM and GDM are often not prescribed insulin and are usually advised to manage their disease through rigorous diet, exercise and oral medication. However, in extreme cases, if the patient is simply unable to control his/her glucose levels, the patient is then prescribed insulin therapy. 
Due to a limitation on the thesis scope, the following section will only discuss the current treatment options for T1DM. Treatment options for other types of DM can be found in Annex A of this document. As explained previously, due to the lack of production of the peptide hormone insulin, a patient afflicted with T1DM needs to inject themselves with insulin on a regular basis. However, the infusion of insulin has to be rigorously monitored since the over-infusion can cause hypoglycemia (a condition when low concentrations of glucose in the blood stream) and under infusion can cause hyperglycemia. Due to this, when manual insulin therapy (via injections) is used, the dosage is based off a blood glucose reading that is taken manually via the finger stick method or automatically via a continuous glucose monitor (CGM). Annex A gives more insights as to how the manual and automatic blood glucose readings are obtained.

In the 1980s however, a novel innovation led to the commercial use of continuous subcutaneous insulin infusion (CSII) pump. This medical device is considered more like a tool, and only delivers exogenous insulin when commanded to or as per a given schedule. In other words, this device is an open-loop control device that facilitates the delivery of insulin but still requires human input.

In order for a patient afflicted with T1DM to treat themselves with insulin therapy, they must first determine their current blood sugar level. It should be noted that, due to physiological reasons, there is no way to know what the exact blood sugar level is at any given time in the whole body. A person can only measure the instantaneous blood sugar level at that particular location in their body. That being said, a person's blood sugar level does not drastically change from one instant to the next unless something with high glucose concentrations is absorbed by the body in between the two instances. Nevertheless, there are currently two methods that are used to measure glucose - continuous glucose monitor (CGM) or a finger prick test. Both methods are 
explained in detail in Annex A.

The CGM is relevant to this thesis as it aids in the development of the 'artificial pancreas'. The CGM is a small intravenous device that is often placed on the abdomen of the patient (preferably as close to the pancreas as possible ${ }^{1}$ ). It's sole function is to measure the blood glucose concentrations continuously. This is to ensure the patient is aware of his/her glucose concentrations and can inject themselves with insulin or ingest something with a high glucose concentration as necessary to maintain their blood glucose levels in the normal range.

\subsection{Literature Overview}

This section is responsible for reviewing some of the current literature that is published and is related to the problem at hand. This section is broken into two main parts: first a physiological model is needed to model a patient with T1DM used to validate the Control Algorithm Device (CAD). Second, is a control algorithm which would be deployed on the CAD as a part of the entire 'artificial pancreas'.

\subsubsection{Metabolic models}

In recent years, due to the huge strive put forth by the research community, a number of metabolic models have been developed and published in literature to capture the dynamics of the insulin-glucose system in the human body. For ease of understanding the models presented in literature currently fall into three groups: empirical models; semi-empirical models; and, physiologically-based models.

\footnotetext{
${ }^{1}$ The blood glucose level can vary at different sites of the body. It is best to measure the blood glucose level where the body naturally measures it (near the pancreas for DM). In addition that manufacturers recommend that the site of monitoring is best where the body does not come in a lot of contact (including natural bending such as a joint or sites that can causes irritation such as the belt line) during regular activity. This is to ensure the CGM stays in adequate contact with the body and thus enhancing its performance [27].
} 
The first group of models, empirical models, is best understood in the realm of computing; engineering; and, science, as black box models. It's sole purpose is to evaluate and capture the transfer characteristics also known as the input and output (glucoseinsulin dynamics) of the system without any consideration for the internal workings (physiology of the human body) of the system [28-31]. The development of these models requires the identification of structures and parameters that are much simpler than the latter two types of models. Thus, the development and synthesis of these models can also be a lot shorter. Since these empirical models are an important step to the development stage of the 'ultimate' controller algorithm design, a number of these well designed empirical models are developed to employ dynamic techniques [32]. These models however cannot be used directly to interface between the CGM and insulin pump in the AP because they pose a number of disadvantages. For example, models that are pure empirical models do not take into consideration any sort of biology. This makes it extremely difficult to distinguish and separate any give specific physiological effects caused by metabolic substrates that occur in any tissue and/or organs. In addition, like any black box system, the models do not provide details into the dynamics of the given system. Lastly, like many biological systems, the dynamics of a glucose-insulin system are non-linear and can vary tremendously given different conditions. Needless to say, these models that depict simple linear insulin-glucose dynamic relationships only provide a good basis to further extend it in order to use it for a control model that would entail more realistic perturbations such as exercise; and, meal intake [28].

Semi-empirical models take it one step further than empirical models. Semi-empirical models still for the most part takes the black box approach to modelling; however, it includes physiology dynamics that are directly related with the system [33-40]. They do this by including many macroscopic metabolic parameters. For example, when 
attempting to design a semi-empirical model for insulin-glucose dynamics one would include parameters such as sensitivity of both insulin and glucose, effectiveness of both insulin and glucose in accordance with tissues that are extra-hepatic (i.e. tissues that are situated outside the liver) [41]. The disadvantage of these models is that they are complex enough to distinguish and separate any give specific physiological effects caused by metabolic substrates that occur at various levels in the human body (i.e. organs vs. tissues vs. cells). This again, is simply due to the fact that the very definition of a semi-empirical model is to depict major physiological interactions while still preserving the simple structure that empirical models are well known for. Hence, these models can be used directly when designing a preliminary CAD that is only capable of maintaining glucose homeostasis without any perturbations such as exercise; and, meal intake.

Lastly, physiologically-based models are the current gold standard when it comes to models pertaining to biological systems. It is the most detailed and complex in terms of the number of parameters, equations and general structure. Different models vary in the depth of physiology that they consider and include sufficient detailed description of the physiology behind the various metabolic interaction involved in the system under consideration ${ }^{2}$ [42-45]. All physiological models capture the metabolic substrate at various levels of the body - from organs, tissues to intracellular levels. It should be noted that for a model to be considered a valid physiologically-based model it needs to describe the dynamics of the system in measurable quantities and correctly predict all the other variables relevant to the system that cannot be measured quantitatively. Thus, these models are extremely useful as they provides sufficient insight into the inner workings of the system. The disadvantage of developing such a complex

\footnotetext{
${ }^{2}$ Many a times newer models are more complex and accurate. A few times newer models are less complex as the authors are able to prove a certain metabolic interaction is not related to the glucose insulin interactions in the human body.
} 
model is that it is time intensive; and, thus often developed by researchers in collaboration over numerous years. Due to the model's complexity and the given nature of biological systems, these physiologically-based models are designed with non-linear equations with numerous parameters.

\subsubsection{Empirical Models}

As mentioned in section 2.3.1 of this document, empirical models are considered to be black box models which only consider the input and output of the system, it does not consider any of the transfer characteristics (in this case physiology) of the system. There are many empirical models that have been developed over the years. However, it is not the aim of this document to discuss the advantages and disadvantages of all of them. Hence, only a handful key empirical models that have revolutionised the development of metabolic models will be discussed.

Using the Volterra-Wiener approach of modelling, a non-parametric model that depict the insulin-glucose interactions was developed in [46]. Using the Laguerre-Volterra Network (LVN) methodology and black box data generated from a parametric model outlined in [33], the first and second order kernels of the Volterra-Wiener model were estimated. This entire model and simulation indicated that it was feasible to develop an accurate nonlinear black box model from insulin-glucose data that resulted from a parametric model. The results from [46] also proved two key things: a Volterra-Wiener modelling approach is robust even in the presence of additive noise; and, similar models can be easily used in the development of a CAD considering that it accommodates its enhancement to include both adaptive and patient specific estimation.

Bremer and Gough in [28] proposed a linear black box model for glucose predictions that is solely based on recent blood glucose measurements. They do this by identifying 
a model structure based on an auto-correlation function (ACF) that estimates blood glucose levels at fixed time intervals. This in turn provides a statistical coefficient of the dependency between each individual measurement of a process at different times. Additionally, the simulation data analysed in [28] demonstrates that blood glucose in not random and actually can be predicted well given frequent sampling. The disadvantage of this model was that it did not permit the prediction of blood glucose for more than 30 minutes.

Finan et al. in [30] developed several empirical models using ARX and Box-Jenkins (BJ) techniques to help evaluate 'infinite step ahead' blood glucose predictions. These models were described in the semi-empirical model that was described by Hovorka et. al in [40]. High accuracy of these models were achieved by higher order and lower order ARX and BJ models under normal operating conditions. However, when trying to simulate a change in parameters such as insulin sensitivity all models were highly inaccurate, especially the low-order models.

A control empirical model that can be used to predict the blood glucose level of a critically ill patients in the intensive care unit (ICU) was developed by [31] by using real clinical insulin-glucose data from an ICU. The following variables were used to predict blood glucose concentrations using an auto regressive exogenous input (ARX) model: adrenalin level; beta-blockers level; body temperature; dobutamine level; dopamine level; glucocorticoids level; insulin infusion rate; total $\mathrm{CHO}$ calorie intake; and, total fat calorie intake. Although the model coefficients proved to have significant relevance to blood glucose, insulin, insulin resistance and $\mathrm{CHO}$ calories; further data is required to enhance the model to ensure it is more patient specific especially since ICU patients often have periodic insulin resistance. Another drawback is that this model requires the use of parameters that cannot be measured continuously and without non-invasive methods. 
With the use of blood glucose concentrations from subcutaneous glucose sensors and subcutaneous insulin infusion pumps a nonlinear neural network (NN) model was developed by Trajonski et al. [47]. This system consists of: the model is based on a nonlinear auto-regressive with exogenous input (NARX) representation; the regularisation approach for constructing radial basis function NNs; and, finally methods of validation include those pertinent to nonlinear systems. Although the closed loop simulation showed that large noise levels did not prevent the system from achieving stable control, rapid insulin control actions did not result in a stable control such as in the OGTT described in section A.4.

Yet another linear black box step response system was developed by Parker et. al in [29]. This model filters the impulse response coefficients through projection onto Laguerre basis and in turn models a step-response effect of insulin in the system. Even though the model does capture a dynamic behaviour of the system; it does not include all the gain information a typical diabetic patient would undergo. It also stated that its failure to capture any steady state condition did not influence the performance of the model. In another study outlined in [48], empirical Volterra series models of insulin-glucose behaviour based on data generated from a physiologically based model [45]. This model accurately predicted data in the absence of noise. However when using an additive Gaussian distributed noise, the coefficient estimates were significantly corrupted. By projecting the Volterra models onto the Laguerre basis functions, considerable amount of noise was filtered. The best performance was achieved by a MPC that filters noise effect after a significant amount of tuning.

Predicting blood glucose levels in T1DM patients using qualitative modelling techniques with fuzzy logical systems has also been proposed by Bellazzi et al. [49]. Prior structural knowledge of the system is used to initialise a fuzzy inference procedure. This procedure predicts a patient's future blood glucose level by approximating the 
system dynamics using the experimental data. Although this methodology can be used accurately and efficiently, the initialisation based on priori knowledge of a qualitative model. Any error in the approximation of this mode could degrade the entire empirical system.

The last empirical model that is considered in this document is that proposed by Bleckert et al. in [50]. This model, defined in $\mathrm{n}$ terms of biological parameters and noise parameters, uses a mixed graphical techniques stochastic linear differential equations to model blood glucose levels in a patient afflicted with DM. In order to handle noise gracefully the model uses density estimates of the unknown parameters which were obtained from the input-output data by using the exact inference algorithm. These density estimates convey much more information about the unknown parameters than a point estimate.

\subsubsection{Semi-Empirical Models}

As mentioned in section 2.3.1, semi-empirical models are the next step up from empirical models. They include only the necessary transfer characteristics of the system. In a biological system this means only the necessary physiological characteristics that directly affect the system. Although there are many semi-empirical models that have been developed over the years; it is not the aim of this document to discuss the advantages and disadvantages of all of them. Thus, only a handful of key semi-empirical models developed by pioneers with be discussed.

The first model that will be outlined was published in 1961 by Boile. This work modelled the insulin-glucose kinetics as a two dimensional model consisting of two Ordinary Differential Equations (ODEs)[34]. One ODE depicted glucose kinetics, while the other depicted insulin. The model also consisted of five parameters that were estimated from various published data. In 1965, Ackerman et al. published a 
similar model. This model took into consideration that both glucose and insulin are in fact interdependent on each other and thus should be modelled in that fashion. A few years later, Serge et al. developed another model that consisted of linear ODEs. This model was said to illustrate insulin-glucose kinetics specifically in obese patients that were diagnosed with T1DM [51]. The main disadvantage with all these preliminary semi-empirical models is the simplification of key physiological effects between glucose and insulin.

The most notable model that resulted in a breakthrough in terms of modelling insulinglucose kinetics was the minimal model that was developed by Bergman et al. [33, 52]. This model used three ODEs that represented plasma glucose dynamics, plasma insulin dynamics; and, insulin concentration. This model was widely used in over 500 studies [53] mainly because it was the first model to accurately model the major physiological metabolic effects of insulin-glucose kinetics. The accuracy of the model parameters during an intravenous glucose tolerance test (IVGTT) was remarkable given its simplicity. However, there are a number of concerns regarding this model that were raised by the research community such as the overestimation of glucose and underestimation of incremental insulin secretion; or, the question as to whether the minimal model itself be too minimal $[38,54,55]$.

Another key group that has developed a number of metabolic models capturing various levels of physiological phenomena is Cobelli et al. [36,37,39,41,56,57]. One of the early models developed by this group tackled one of key disadvantages of the model suggested by Bergman - the separation of glucose utilisation and production. The next notable publication included the most sought after solution at the time. One that solved the argument of overestimation of glucose and underestimation of insulin in the Bergman model. The next series of publication improved the models by adding two key hormones that directly affect the insulin-glucose system in the human body - 
insulin and glucagon (a hormone secreted by the $\alpha$-cells of the pancreas that encourages the liver to increase the production of glucose). Hovorka et al. then extended this model by adding separate insulin components. This new compartmentalised model consisted of a plasma insulin; two glucose sub-systems; and, three separate remote insulin compartments which captured other various physiological effects. The biggest issue with this model was the absence of steady state functions which failed to predict cases of high insulin ingestion [40].

The next model under consideration is one described in [58]. This particular model is best known for its simulation of insulin-glucose dynamics after a subcutaneous insulin injection. The simulation can accurately predict a change in plasma glucose concentrations due to small meal intakes (i.e. CHO) or insulin absorption. It does this with the help of a pharmacodynamics model with parameters from literature. However, the limitations of the model were clearly seen when it attempted to examine the effects of a big meal intake.

A biological relevant model describing the in vivio insulin-glucose physiological system in T1DM was developed by Salzsieder et. al in [59]. It consisted of four state variables that served as: glucose; insulin; net endogenous glucose balance; and, peripheral insulin-dependent glucose uptake. The model validation was published in [59-61] and proved that the slow dynamics and steady state glucose dynamics were accurately represented. There are two major disadvantages of this model. The first, is the limitation in linearity which focuses on the high glucose levels as opposed to the frequent low blood glucose levels seen in T1DM patients. The second is the absence to examine the slow insulin dynamics.

A semi-empirical insulin-glucose model was also proposed by Boroujerdi et al. and is documented in [62]. This models breaks down the glucose utilisation into two processes (insulin dependent and independent) which is different from that of previously 
discussed models. This five compartmentalised model (insulin insensitivity, insulin sensitivity, plasma glucose, glucose interstitial space; and, glucose transporter with a limited metabolism compartment for each of the interstitial space) proved that hyperglycemia in DM patients is caused by the saturation of the glucose transport mechanism. This remarkable discovery ensured the modelling and simulation community gained a lot of headway. When it comes to accurately modelling the insulin-glucose kinetics it also proved that most models do not tackle the behaviour of insulin on endogenous glucose production when tissue saturation occurs in the human body.

Another glucose-insulin modelling effort can be seen in the published works of Lehman and Deutsch [63].The problem that was tackled in this work was simulating insulin response (both plasma and glycemic) for 24 hours in order to evaluate patients afflicted with T1DM. It accomplishes this by simulating glucose entering the system via hepatic glucose production and intestinal absorption. Glucose leaves the system only under three conditions: glucose utilisation in red blood cells (insulin independent); glucose utilisation through the rest of the cells in the body (insulin dependent); and, excretion of glucose through the renal system in the human body. Since its publication, this model has been widely used to assist in designing treatment options for patients afflicted with T1DM [64]. The biggest limitation of this model was discussed by Steil et al. in [65] who argues that the model cannot accurately predict glucose dynamics during steady state insulin infusions.

A semi-empirical capable of capturing insulin-glucose dynamics and regulation based insulin pulsating secretion was captured by Tolic et al. in [66]. It includes a feedback look that consists of four components: pancreatic insulin secretions based on glucose stimulation; glucose uptake based on insulin stimulation; suppression of hepatic glucose production; and, glucose uptake based on its own enhancement. Another key 
aspect of this model is the incorporation of delays which mimic the physiological delays seen in humans when examining the insulin-glucose dynamics. However, as the authors acknowledged, at the time of publication, the model was in its early stages of development and needs to be refined quite a bit in order to incorporate a more realistic simulation of the various states the human body goes through especially during the time delay aspect of the model. In addition, the model only takes into account the effect on insulin receptors on the upward cycle of the pulsating insulin secretion. It does not take into account the effect the downward cycle has on the insulin receptors.

Another limitation with Bergman's minimal model was that it was developed with the intent of capturing insulin-glucose dynamics only of a single individual [33]. However, since these metabolic models are often used to develop a CAD, it is important that these models are able to accurately depict an entire population. In order to tackle this limitation Vicini and Cobelli proposed a population-based minimal model that used the Bayesian approach in [67]. Using the IVGTT data from sixteen individuals who were not afflicted with the disease, they examined the distribution of insulin sensitivity; and, glucose effectiveness for a population. With this approach, the model could estimate both the insulin sensitivity; and, glucose effectiveness accurately even in a small sample size. The issue with this model is its simplicity - modelling only glucose dynamics of the system is not nearly enough. Andersen and Hojbjerre in [68] employed a very similar stochastic minimal model which included both insulin and glucose kinetics. The advantage of this method was that it accounted for a lot of error in both the measurement and dynamics processing. They did this by employing a Markov chain Monte Carlo approach to estimate the parameters using the Bayesian technique.

Using two delayed differential equation (DDE) for modelling insulin-glucose dynamics of a patient connected to an exogenous insulin delivery system was developed by 
Engelborghs et. al in [69]. The model incorporated two delays: the physiological delay which accounted for the delay that insulin had on the production of glucose in the human body; and, the delay associated with the exogenous insulin delivery system. The analysis of this work proved that the two major types of diabetic patients (T1DM and T2DM) had very different efficiencies when it came to insulin delivery.

Another semi-empirical model effort was demonstrated by Kraegen and Chisholm in [70] which modelled the continuous subcutaneous insulin infusion using a kinetic model. This two compartmental model demonstrated a key aspect of insulin infusion therapy: subcutaneous insulin absorption in extremely low. A three compartmental model directly linking the insulin dosage level and blood insulin levels was then developed by Puckett and Lightfoot in [71]. A study comparing the two fore mentioned models revealed that data extracted from DM patients' data was better fitted by the results from the latter model. The study also proved that there was a significant improvement in the results from the three compartmental model when analyzing the results for variability with respect to a patient's response to insulin over time (interpatient variability) and intra-patient variability.

The last semi-empirical model that will be considered is the one proposed by Wach et al. in [72]. Using partial differential equation (PDE), this model was developed with the intent of depicting three aspects: the diffusion of soluble insulin on the human body; the steady state dynamics between monomeric and dimeric insulin; and, the absorption of monomeric insulin molecules. The PDEs were solved using various numerical methods and division of subcutaneous regions into smaller spherical spaces to account for the space distribution in cells. The model results did fit with experimental data and could be used in a clinical setup. 


\subsubsection{Physiological Models}

Just like the previous two sections, this section will only describe a handful of relevant physiological models. A physiological model as described in section 2.3 .1 is one that takes into account a number of different parameters, equations and the structure of the human body. The first model that will be considered was proposed in the mid70s but Tiran et al. $[42,73]$. Dubbed as the circulation model, it is often accredited as the first physiological model to accurately depict insulin-glucose kinetics in the human body. It consisted of a number of different compartments that represented major organs and tissues in the human body; with each compartment divided into two sub-compartments: one representing the capillaries and the other representing the interstitial space. Additionally, the model also took into account the non-linearity of glucose metabolism in the human body. This model in particular performed quite well when tested in response to glucose ingested in small boluses. However, any glucose intake that is greater than 30 grams was over-predicted. This preliminary model also did not take into account any metabolic behaviour near the thresholds.

Cobelli et al. in [44] also proposed a semi-physiological non-linear model that depicted insulin-glucose dynamics. The model consisted of functions that captured insulin degradation; insulin secretion; glucose absorption as a result of a meal intake; glucose consumption by peripheral organs and tissues; hepatic glucose production and utilisation; and, renal glucose excretion. The incorporation of delay with respect to glucose regulation and the effect of insulin on glucose also contributed to successful depiction of a normal human body after a meal was ingested. The model however lacked taking into account the distribution of glucose in many major glucose-consuming organs.

Based on a model by Guyton et al. [74], a physiologically-based metabolic model was developed by Sorensen [45]. The model described the insulin-glucose dynamics by utilising 19 differential equations: eleven of which were used to describe the glucose 
system, seven described the insulin system and one described the glucagon system. The glucose sub-model consisted of various compartments that represented various parts of the body's organs and tissues. Some of these compartments were further divided to account for capillary and interstitial spaces which accounted for the resistance of the human body with respect to the glucose transport mechanism. In addition, metabolic sinks were represented throughout the model to account for the glucose uptake of various tissues and organs. The insulin sub-system was modelled in a similar fashion with one key difference - insulin had only one sub-compartment in the brain and this was to account for the physiological phenomena of insulin not entering the brain capillaries [33]. Various studies have been conducted to validate the model. All these studies have concluded that this model accurately predicts the concentrations of plasma glucose level in the human body after IVGTT.

Another physiologically based model was developed by Kim et al. [75]. This metabolic model also captured the whole body homeostasis during exercise. The model is also a compartmentalized model that divided the body into adipose tissue (AT); gastrointestinal tract; heart; liver; muscle; and, other tissue. This was achieved by using two aspects which were modelled for each compartment: dynamic mass balance and cellular metabolic processes. The model was then validated with experimental data in which normal healthy individuals were asked to perform moderate intensity exercises for an hour. This physiological model accurately captured the insulin-glucose dynamics. This novel approach can now be used to model and study the effects of hormone control and metabolic concentrations in various tissues of the human body. However, the model lacked in validating the same effects on patients afflicted with DM.

All the models discussed so far span approximately five decades and only take into account the dynamics between insulin and glucose on the human body. However the 
effect of free fatty acid (FFA) and exercise on this system have been for the most part disregarded. In order to combine both high quality and robustness of a biological model based on a human body, it is essential that the models takes into account disturbances such as a mixed meal ingestion and various levels of exercise. However, to this day there are but a few models that accurately describe this behaviour in a physiologically accurate method. Considering all this, the best known model that is used widely to validate many CAD is known as the Dalla Man model. As its name suggests, the model was proposed by Dalla Man et al. in a paper titled "Meal simulation model of the glucose-insulin system" [76]. It is currently the gold standard for validating CDA without conducting pre-clinical animal trials that is accepted by the FDA [77]. This model not only presented information such as the concentrations of insulin and glucose in the plasma; but also; endogenous glucose production; glucose utilisation; glucose rate of appearance and, insulin secretion. The model was validated by monitoring a large population (204 healthy individuals and 14 individuals afflicted with DM). The model parameters included were in two sets: the first included parameters for healthy individuals and the second included parameters for patients with DM. During validation of the model the meal ingested was labelled with radioactive materials such that the flow of glucose through the gastrointestinal tract could be accurately measured. Additionally, upon ingestion, two other tracers were injected intravenously, the data then used to estimate model parameters as explained in [78]. Using this triple tracer meal protocol, the following four fluid flows were estimated: rate of appearance (Ra); endogenous glucose production (EGP); glucose utilisation; and, insulin secretion.

\subsubsection{Control Algorithm Devices (CAD)}

As discussed in section 2.2, an artificial pancreas would ideally be the 'cure' for diabetes mellitus. Klonoff in [79] defined the artificial pancreas as one that consisted 
of three components: a CGM; an insulin pump; and, a CAD. This methodology of monitoring glucose; accurate determination of insulin dose by the CAD and delivery of insulin dosage by the insulin pump could result in a patient with DM always having normal physiological glycemic levels.

In 1964, Kadish was accredited with the first closed loop insulin delivery system - a simple on-off system [80]. This system was designed to measure blood glucose levels every 15 seconds and based on its results would deliver glucagon (blood glucose levels are too low - i.e. $\leq 50 \frac{m g}{d L}$ ) or, insulin (blood glucose levels are too high - i.e. $\geq 150$ $\left.\frac{m g}{d L}\right)$. As a result the blood glucose of the individual always remained in the range of 50-150 $\frac{\mathrm{mg}}{\mathrm{dL}}$. Due to the lack of technological advances, this device was as big as a backpack and the lack of computational power severely limited the performance of this device.

Another likely candidate for the AP was developed by Albisser et al. and documented in $[81,82]$. This two channel device (one containing dextrose insulin and the other used for the infusion of insulin into the human body) included a CGM and control algorithm which were both deployed on a microcomputer. This device posed two major problems: the control algorithm required several specific highly dependent patient parameters and the response to a glucose spike (i.e. meal ingestion) was gradual.

The development of the AP known as the Biostator was developed by Miles Laboratory and was commercially available in 1977 for a period of time [83]. The CAD included in this device employed a non-linear proportional derivative (PD) control algorithm that regulated both dextrose and insulin infusions in order to maintain homeostasis. The controller injected insulin based on the regular measurement of plasma glucose concentrations. Noise of this measurement was filtered using a five 
point moving average. However, this device was soon pulled out from the commercial market because it required the individual to constantly monitor it. Additionally, other disadvantages included but are not limited to it's size; and, parameter individualization.

Based on the work of Albisser et al.[42] several other control algorithms were proposed. These included, but are not limited to: Botz [84]; Kragen et al. [85]; and, Marliss [86]. All these models attempted to reduce the hyperglycemic event caused by the initial model after ingestion of a meal; or, the hypoglycemic event caused after exercise. However, Broekhuyse in [87] reviewed all these models and concluded that neither were even close in accuracy nor should not be implemented in a commercially available AP.

The extensive studies carried out over a number of year by Fischer et al. [60] led to the development of three different control strategies to control blood glucose levels in patients afflicted with T1DM. This was the direct result of initially concluding that an adaptive algorithm would guarantee an optimal feedback methodology for controlling blood glucose levels. Their models were tested on diabetic dogs who were maintained at rest (i.e. no exercise to reduce blood glucose levels). The first control algorithm was implemented by an adaptive control algorithm that was sampled at 1 minute intervals. The effects were observed on an insulin-glucose system for four hours. The second control algorithm employed a fixed command control that was initialized by estimates. Lastly, the third control algorithm, a modification to the second, was also a fixed command control algorithm that was individualized. The estimates and control constants that this algorithm used as modified based on prior knowledge and tests conducted on the patient. When the three models were evaluated, it was proven that only the first and second achieved glycemic levels that were in the normal range.

An external physiological insulin delivery system (ePID) was developed by Medtronic 
Minimed [88]. The design intent was to emulate the $\beta$-cells of a normal pancreas such that the control algorithm would distinguish and control the first and second phase insulin secretion $[89,90]$. The problem was tackled by using a proportional integral derivative (PID) controller [91]. The first phase of insulin secretion was linked to the derivative aspect of the controller and is directly tied in with the rate of change in blood glucose levels as read by the CGM. The second phase is controlled by the proportional and integral aspect of the controller by establishing a direct link between the target and the actual blood glucose concentrations. Although an evaluation of this CAD resulted in satisfactory results it cannot prevent hyperglycemic events that occur as a direct result of meal intake [88].

Another promising methodology was the optimal controller ${ }^{3}$ that was developed by Swan [92] who estimated the insulin infusion rate by solving a non-linear algebraic Riccati equation. The model cannot be confirmed due to the fact that its performance after meal ingestion was not evaluated. An extension to Bergman's minimal model was implemented by Ollerton in [93]. Based on a deviation from the target glucose value, Ollerton developed an integral squared error objection function. The CGM however was sampled at a rate of ten minutes so that the algorithm calculation time was minimized. However this CAD was criticized when it was discovered that the algorithm would not converge at the basal state, instead, it would oscillate significantly. In another study conducted by Fisher and Teo [94] various insulin infusion protocols were tested in the quest to find an objective function that would be minimized. The study concluded by applauding the sum-squared error set-point tracking methodology as the most accurate model for maintaining blood glucose levels during meal intake and fasting. However, this model seemed to only thrive provided a good

\footnotetext{
${ }^{3}$ The methodology is known as the optimal controller because uses optimal control theory and solution of a nonlinear algebraic Riccati equation to solve the glucose control problem for the optimal insulin infusion rate.
} 
meal estimation was available.

Since 2009, there have been a number of CADs developed using model predictive controllers (MPC). The approach used is true to its name, using a model based control approach to control the blood glucose levels in a patient. In general it consists of the model; the cost function; and, the constraints. The model itself is used to predict the future of the system (states and/or outputs) based on its current state, estimation of future values that are attained from manipulating variables; and predictions of future values of any measurable or predictable disturbances. It's flexible in the sense that the model can be of any kind - based on continuous time or discrete time; linear or nonlinear; input-output or state-space; black box, grey box or white box models. The cost function aspect is a closed loop aspect, and is usually based on a differential equation of degree two. Lastly, the constraints are based on the controlled and manipulated variables [95]. It has been proven that using an MPC to define the CAD has many advantages in terms of regulating blood glucose levels in patients afflicted with T1DM. The biggest advantage can be seen in the model prediction characteristic of the MPC which can foresee a patient going into either hyperglycemic or hypoglycemic state and within reason can adjust itself to compensate for it. Additionally it also compensates quite well for the dynamics associated with the equipment used - i.e. the insulin infusion pump and the CGM [65]. Lastly, the MPC can be constricted which permits the insulin infusion rate to be bound which accounts for the safety aspect that is lacking in many other CAD [96].

A MPC was developed by Trajonski et al. [47], in an attempt to regulate blood glucose in individuals afflicted with DM. This system used subcutaneous glucose measurement and insulin infusion. The control algorithmic part that predicted glucose concentrations was defined by a radial basis function (RBF) neural network model. This was done by using past subcutaneous glucose measurements and insulin infusion 
rates. Even in the presence of noise, a simulation of this model indicated that the blood glucose levels of a patient could be controlled and stabilized.

The next MPC that will be considered was proposed by Parker et al. in [29]. It is a linearized adaptation of Sorensen's physiologically-based model with and without state estimation. The constraints aspect of MPC was applied on the rate of change of insulin infusion and rate of input delivery. A simulation modelling both a nonlinear Sorensen physiological model and the MPC in question was performed in order to validate the results. It concluded that normal glycemic levels were maintained after a 50 gram OGTT. Another physiologically based MPC can be seen in the works of Lynch and Bequette [97] who developed the MPC on the basis of the extension of the minimal model by Fisher [98]. The model used state estimation of subcutaneous blood glucose measurements. The validation of this model was performed when simulating a high order Sorensen model. It was concluded that with proper tuning, the MPC would return blood glucose levels of a patient to levels within normal glycemic range even after a meal consumption.

To model and examine the effects of fasting in T1DM patients, a MPC was developed by Hovorka et al. [99]. It included a compartmentalized model that was documented in [40]. This model contained time varying parameters which were quantized by the use of Bayesian parameter estimation. In order to normalize blood glucose levels, the CAD also added the gradual decreasing set-point trajectory aspect to the existing model. The system was designed to sample the blood glucose levels and infuse insulin at a rate of 15 minutes. Clinical evaluations were conducted for this controller which validates the effects of insulin infusion on ten patients over a period of 8-10 hours. The study concluded that the adaptive nonlinear MPC proved to be promising and must be further developed to take into account sudden spikes in blood glucose levels due to meal ingestion. 


\subsection{Problem Statement}

In order to understand and design something that acts just like any organ such as the pancreas, a number of research groups must be involved. The complex behaviour is broken down into various smaller sub-components and studied. The knowledge of these partial sub-systems are then combined together to form an artificial organ. The amalgamation of sub-components mimics a hierarchical model structure with multiple levels. The top level represents the whole body, thus representing blood flow between the organs (which includes the flow of glucose and insulin). What is more important is the fact that this hierarchical model allows for model re-use and ease of interchangeability. That is to change the sub-component of the model (if more information of the sub-component becomes available) without actual editing the entire model itself.

This thesis will focus on implementing two different models. The first is the Dalla Man model which is currently the gold standard for mixed meal simulations. This model will act as a 'patient's body' and depict the interactions of glucose and insulin in various parts of the human body. The Dalla Man model will be validated against literature. The second is an adaptation of the Hovorka model. This model will be implemented in DEVS as the and will act as the CAD. The Hovorka model will be validated by using the outputs of the Dalla Man model. 


\section{Chapter 3}

\section{Model Design}

The creation of an artificial system requires a designer to have two major components: knowledge of the system itself and experimental data in order to verify the artificial system [100]. Furthermore, in order to accurately model any dynamic system, the use of time dependent ordinary differential equations is almost compulsory [101]. The following two models are derived from experimental systems and knowledge. They use a number of ordinary differential equations to simulate the details of a human body's glucose regulatory system.

This thesis develops two models (as seen in Figure 6): the physiological system (also referred to as the meal model simulation) and the artificial pancreas [102]. As mentioned in Chapter 1 and Chapter 2, the CGM and the insulin infusion pump have already been developed and are readily available on the market. Thus the only component that needs to be developed is the CAD. This is done via a MPC. The block diagram for this second model is depicted in Figure 7.

\subsection{Meal Model Simulation}

Due to the reasons stated in section 2.3.1.3 of this document, the Dalla Man model was chosen as the meal model simulation that would in turn validate the CAD. The 


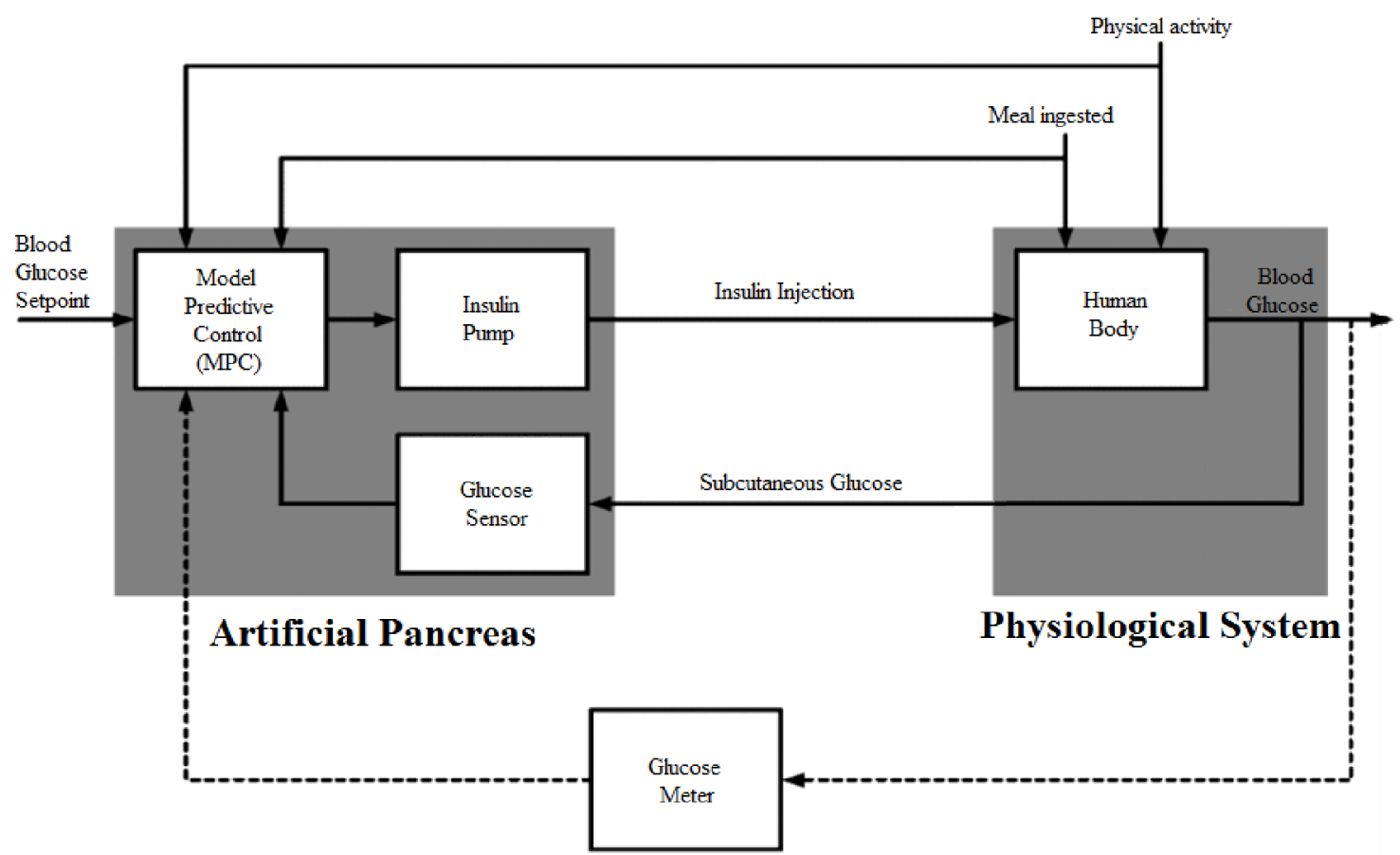

Figure 6: Block Diagram showing the Artifical Pancreas and Physiological system (adapted from [102]). For more details refer to related image depicted in Figure 7.

Dalla Man model is a non-linear compartmental model that acts as a virtual patient. Compartmental models are often widely used in the study of kinetics of materials in physiological systems. They are also dynamic in the sense that they used differential equations that are derived from the basic principles of physics - conservation of mass; and, conservation of energy. A compartmental model is a model that specifies the interconnections between a finite number of compartments. When looking at it from a physiological aspect, these interconnections represent the flow of materials from one compartment to the next.

Since this model was simulated on a hierarchical format, for ease of understanding, the creation of this model will be outlined in a top-down approach. The top most level, as seen in Figure 2 is comprised of various coupled models. At the top most level, a coupled model describing the whole mixed meal model system is created. The inputs 


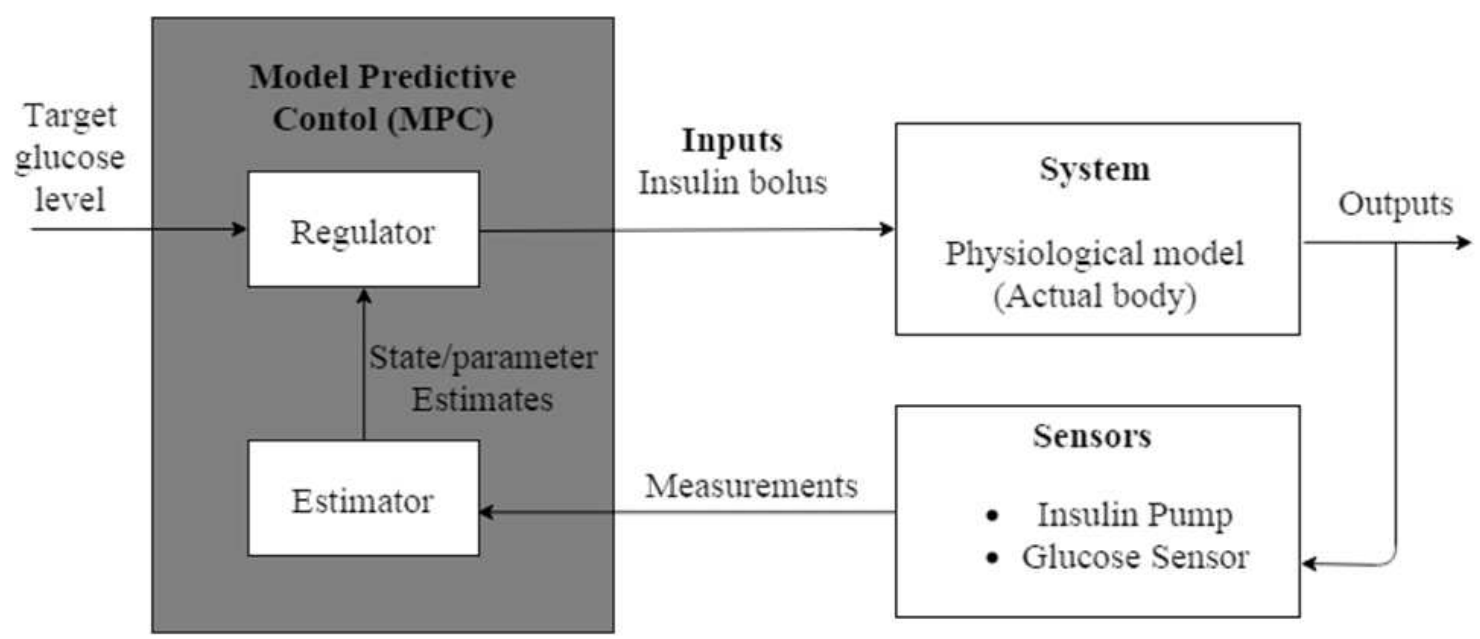

Figure 7: Block Diagram showing the details of MPC (adapted from [102]). For more details refer to related image depicted in Figure 6.

to this coupled model is the meal (amount of carbohydrate intake in grams) itself; similarly, the outputs include: glucose utilisation; and, insulin secretion. Within this coupled model exists two atomic models (depicted on the right in Figure 2) and three coupled models. The atomic models include he gastrointestinal tract and the beta cell. The three coupled models (depicted on the left in Figure 2): the plasma; the liver; and, the muscle and adipose tissue.

\subsubsection{Plasma}

The plasma model can be considered to be the heart of the entire Dalla Man model and is the most integral part with respect to diabetes. It is this coupled model that is responsible for dictating; and, estimating the blood glucose level of the body. This coupled model consists of one atomic model - glucose renal excretion defined by the variable $\mathrm{E}(\mathrm{t})$; and, two integral internal calculations: glucose kinetics; and, insulin kinetics. The glucose renal excretion is the excretion of glucose by the kidneys. This occurs only when the plasma glucose exceeds a certain threshold; and, thus is 
modelled by a linear piecewise relationship with respect to the plasma glucose:

$$
E(t)= \begin{cases}k_{e 1} \cdot\left[G_{p}(t)-k_{e 2}\right] & \text { if } G_{p}(t)>k_{e 2} \\ 0 & \text { if } G_{p}(t) \leq k_{e 2}\end{cases}
$$

Where:

$E(t)$ refers to the glucose renal excretion (defined in $\mathrm{mg} /(\mathrm{kg} \cdot \mathrm{min})$ );

$G_{p}(t)$ refers to the glucose concentration in the plasma at time $\mathrm{t}$ (defined in $\mathrm{mg} / \mathrm{kg}$ );

$k_{e 1}$ refers to the glomerular filtration rate (defined in $\mathrm{min}^{-1}$ ); and,

$k_{e 2}$ refers to the renal threshold for glucose (defined in $\mathrm{mg} / \mathrm{kg}$ ).

The glucose kinetic calculation is comprised of one overall function that is dependent on two sub-functions that are defined in the internal transition of the plasma coupled model: glucose masses in the plasma; and, glucose in rapidly-equilibrating tissues. The overall function (one of the output functions) is defined as:

$$
\begin{aligned}
& G(t)=\frac{G_{p}(t)}{V_{G}} \\
& G(0)=G_{b}
\end{aligned}
$$

Where:

$G_{(t)}$ refers to the plasma glucose concentration at time $\mathrm{t}$ (defined in $\mathrm{mg} / \mathrm{kg}$ );

$G_{p}(t)$ refers to the glucose mass in the plasma at time $\mathrm{t}$ (defined in $\mathrm{mg} / \mathrm{dl}$ );

$V_{G}$ refers to the distribution of glucose (defined in $d l / k g$ ); and, $G_{b}$ refers to the basal steady-state of the glucose mass in the plasma. 
The glucose masses in the plasma is governed by the equation:

$$
\begin{aligned}
& \dot{G}_{p}(t)=E G P(t)+R a(t)-U_{i i}(t)-E(t)-k_{1} \cdot G_{p}(t)+k_{2} \cdot G_{t}(t) \\
& G_{p}(0)=G_{p b}
\end{aligned}
$$

Where:

$G_{p}(t)$ refers to the glucose mass in the plasma at time t (defined in $\mathrm{mg} / \mathrm{kg}$ ); $E G P(t)$ refers to the endogenous concentration at time $\mathrm{t}($ defined in $\mathrm{mg} / \mathrm{kg} / \mathrm{min}$ ); $R a_{t}$ refers to the glucose rate of appearance in the plasma at time $\mathrm{t}$ (defined in $\mathrm{mg} / \mathrm{kg} / \min )$

$U_{i i}(t)$ refers to the insulin independent glucose utilisation at time $\mathrm{t}$ (defined in $\mathrm{mg} / \mathrm{kg} / \min )$;

$U_{i d}(t)$ refers to the insulin dependent glucose utilisation at time $\mathrm{t}$ (defined in $\mathrm{mg} / \mathrm{kg} / \min )$

$G_{t}(t)$ refers to the glucose mass in rapidly-equilibrating tissues (defined in $\mathrm{mg} / \mathrm{kg}$ ); $k_{1}$ refers to the rate of glucose mass in the plasma (defined in $\mathrm{min}^{-1}$ ); $k_{2}$ refers to the rate of the rapidly-equilibrating tissues (defined in $\mathrm{min}^{-1}$ ); and, $G_{p b}$ refers to the basal steady-state of the glucose mass in the plasma (defined in $m g / k g)$.

The glucose masses in the plasma is governed by the equation:

$$
\begin{aligned}
& \dot{G}_{t}(t)=-U_{i d}(t)-k_{1} \cdot G_{p}(t)+k_{2} \cdot G_{t}(t) \\
& G_{t}(0)=G_{t b}
\end{aligned}
$$

Where:

$G_{t}(t)$ refers to the glucose mass in rapidly-equilibrating tissues (defined in $\mathrm{mg} / \mathrm{kg}$ ); $G_{p}(t)$ refers to the glucose mass in the plasma at time $\mathrm{t}$ (defined in $\mathrm{mg} / \mathrm{kg}$ ); 
$U_{i d}(t)$ refers to the insulin dependent glucose utilisation at time $\mathrm{t}$ (defined in $\mathrm{mg} / \mathrm{kg} / \mathrm{min})$;

$k_{1}$ refers to the rate of glucose mass in the plasma (defined in $\mathrm{min}^{-1}$ );

$k_{2}$ refers to the rate of the rapidly-equilibrating tissues (defined in $\mathrm{min}^{-1}$ ); and,

$G_{p b}$ refers to the basal steady-state of the glucose mass in the rapidly-equilibrating tissues(defined in $m g / k g$ ).

The second major calculation that is performed is the insulin kinetics. Like the glucose kinetic calculation, the insulin kinetics is comprised of one overall function that is dependent on two sub-functions that are defined in the internal transition of the plasma coupled model: insulin masses in the plasma; and, insulin mass in liver. The overall function (one of the output functions) is defined as:

$$
\begin{aligned}
& I(t)=\frac{I_{p}(t)}{V_{I}} \\
& I(0)=I_{b}
\end{aligned}
$$

Where:

$\left.I_{(} t\right)$ refers to the plasma glucose concentration at time $\mathrm{t}$ (defined in $\mathrm{mg} / \mathrm{kg}$ );

$I_{p}(t)$ refers to the glucose mass in the plasma at time $\mathrm{t}$ (defined in $\mathrm{mg} / \mathrm{dl}$ );

$V_{I}$ refers to the distribution of glucose (defined in $\mathrm{dl} / \mathrm{kg}$ ); and,

$I_{b}$ refers to the basal steady-state of the glucose mass in the plasma.

The insulin masses in the plasma is governed by the equation:

$$
\begin{aligned}
& \dot{I}_{p}(t)=-\left(m_{2}+m_{4}\right) \cdot I_{p}(t)+m_{1} \cdot I_{l}(t) \\
& I_{p}(0)=I_{p b}
\end{aligned}
$$


The insulin masses in the liver is governed by the equation:

$$
\begin{aligned}
& \dot{I}_{l}(t)=-\left(m_{1}+m_{3}(t)\right) \cdot I_{l}(t)+m_{2} \cdot I_{p}(t)+S(t) \\
& I_{l}(0)=I_{l b}
\end{aligned}
$$

\subsubsection{Liver}

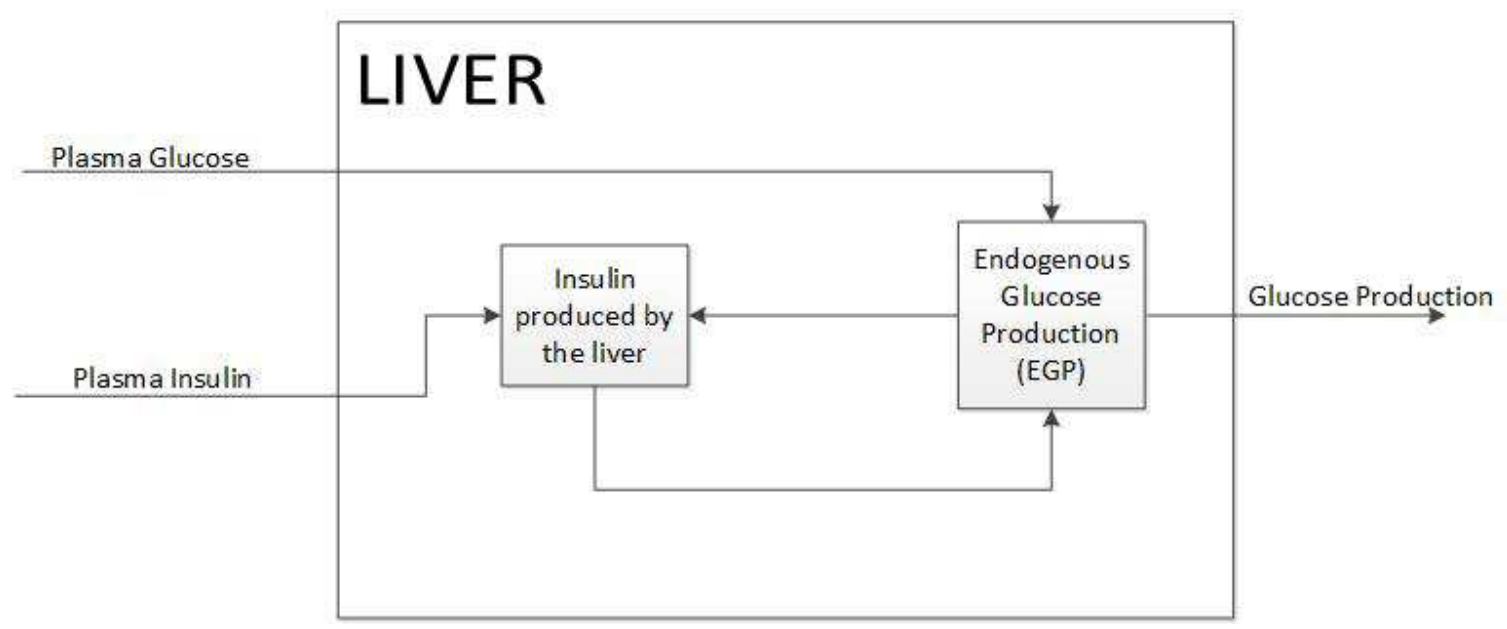

Figure 8: Coupled model representing the Liver.

The liver coupled model defined by the Endogenous Glucose Production (EGP) reactions (see Figure 8) contains three atomic models - insulin in the portal vein; insulin in the liver; and, four inputs:

- $I_{p o}$, the amount of insulin in the portal veins of the patient

- $I_{d}$, the delayed insulin signal

- $I$, concentration of insulin in the plasma

- $G_{p}$, mass of glucose in plasma and in tissues 
The first atomic EGP model is the 'brains' of the EGP coupled model which is mainly governed by a functional description:

$$
\begin{aligned}
& E G P(t)=k_{p 1}-k_{p 2} \cdot G_{p}(t)-k_{p 3} \cdot I_{d}(t)-k_{p 4} \cdot I_{p o}(t) \\
& E G P(0)=E G P_{b}
\end{aligned}
$$

The second and third EGP atomic models act as slaves to the first. The second atomic model defines the delayed insulin signal that is defined by the ordinary differential equation:

$$
\begin{aligned}
& \dot{I}_{1}(t)=-k_{i} \cdot\left(I_{1}(t)-I(t)\right) \\
& I_{1}(0)=I_{b}
\end{aligned}
$$

Lastly, the third atomic model defines the insulin in the portal veins and is driven by the differential equation:

$$
\begin{aligned}
& \dot{I}_{d}(t)=-k_{i} \cdot\left(I_{1}(t)-I(t)\right) \\
& I_{d}(0)=I_{b}
\end{aligned}
$$

\subsubsection{Muscle and Adipose tissue}

The muscle and adipose tissue compartment is responsible for describing the glucose utilisation during a meal in a human body. This is done via two atomic models: insulin-independent and insulin-dependent. The output of the coupled model is simply defined as the summation of the results from the two atomic models:

$$
U(t)=U_{i i}(t)+U_{i d}(t)
$$


The insulin-independent utilisation models the glucose uptake by the brain and erythrocytes $\left(F_{c n s}\right)$. It is modelled by the equation:

$$
I_{i i}(t)=F_{c n s}(t)
$$

The insulin dependent utilisation however, is non-linear and is based on the MichaelisMenten reaction. It is the most complicated atomic model in the Dalla Man model. The equation that governs this atomic model:

$$
U_{i d}(t)=\frac{V_{m}(X(t)) \cdot G_{t}(t)}{K_{m}(X(t))+G_{t}(t)}
$$

Where,

$$
\begin{gathered}
V_{m}(X(t))=V_{m}+V_{m x} \cdot X(t) \\
K_{m}(X(t))=K_{m}+K_{m x} \cdot X(t)
\end{gathered}
$$

$V_{m}$ us a function of insulin that describes the maximal uptake present near any given cell (represented as X). The more insulin present in the blood stream, the more glucose any given cell is able to intake through it's membrane. $K_{m}$ is the Michaelis-Menten constant; and, $G_{t}$ is the saturation of glucose in the plasma.

\subsubsection{Gastrointestinal Tract}

The gastrointestinal tract coupled model is used to describe the glucose that transits through the stomach and the intestines of the person. This coupled model is divided 
into three atomic models. Similar to the liver coupled model, an atomic model is used as master of the gastrointestinal tract component, and the other atomic models.

The main atomic model is governed by the functional description:

$$
\begin{aligned}
& R a(t)=\frac{f \cdot k_{a b s} \cdot Q_{g u t}(t)}{B W} \\
& R a(0)=0
\end{aligned}
$$

The second atomic model, describes the intestines/gut of the human body. It is driven by the following equation:

$$
\begin{aligned}
& \dot{Q}_{g u t}(t)=-k_{a b s} \cdot Q_{\text {gut }}(t)+k_{\text {empt }}\left(Q_{\text {sto }}\right) \cdot Q_{\text {sto2 }}(t) \\
& Q_{\text {gut }}(0)=0
\end{aligned}
$$

The last atomic model describes the stomach of the human body. It is driven by two differential equations and one output equation. The two differential equations are described and calculated as internal transitions.

$$
\begin{aligned}
& Q_{s t o}(t)=Q_{s t o 1}(t)+Q_{s t o 2}(t) \\
& Q_{s t o}(0)=0
\end{aligned}
$$

The first differential equation describes how the stomach process its contents in the solid phase:

$$
\begin{aligned}
& \dot{Q}_{s t o 1}(t)=-k_{g r i} \cdot Q_{s t o 1}(t)+D \cdot \delta(t) \\
& \dot{Q}_{s t o 1}(0)=0
\end{aligned}
$$


The second differential equation describes how the stomach processes its contents that are triturated phase:

$$
\begin{aligned}
& \dot{Q}_{s t o 2}(t)=-k_{\text {empt }}\left(Q_{\text {sto }}\right) \cdot Q_{\text {sto } 2}(t)+k_{\text {gri }} \cdot Q_{\text {sto } 1}(t) \\
& \dot{Q}_{s t o 2}(0)=0
\end{aligned}
$$

Note that the rate constant of gastric emptying that is part of the titurated phase of the stomach and the intestinal atomic model is defined as:

$$
k_{\text {empt }}\left(Q_{\text {sto }}\right)=k_{\text {min }}+\frac{k_{\max }-k_{\min }}{2} \cdot\left\{\tanh \left[Q_{\text {sto }}-b \cdot D\right]-\tanh \left[c \cdot\left(Q_{\text {sto }}-d \cdot D\right]+2\right\}\right.
$$

\subsubsection{Beta Cells}

The beta cells in the model are responsible for modelling pancreatic insulin secretion. The atomic model is governed by the overall equation:

$$
S(t)=\gamma \cdot I_{p o}(t)
$$

There are three functions defined in the internal transition function of the beta cell atomic model: insulin in the portal $\left(I_{p o}(t)\right)$; insulin secretion to the portal vein $\left(S_{p o}(t)\right)$; and, the insulin response to cells $(Y(t))$. These three internal functions are described by the following three sets of equations respectively:

$$
\begin{aligned}
& \dot{I_{p o}(t)}=-\gamma \cdot I_{p o}(t)+S_{p o}(t) \\
& I_{p o}(0)=I_{p o b}
\end{aligned}
$$




$$
\begin{gathered}
S(t)= \begin{cases}Y(t)+K \cdot \dot{G(}(t)+S_{b} & \text { if } \dot{G}>0 \\
Y(t)+S_{b} & \text { if } \dot{G} \leq 0\end{cases} \\
Y(t)= \begin{cases}-\alpha[Y(t)-\beta \cdot(G(t)-h)] & \text { if } \beta \cdot(G(t)-h) \geq-S_{b} \\
-\alpha \cdot Y(t)-\alpha \cdot S_{b} & \text { if } \beta \cdot(G(t)-h)<-S_{b}\end{cases} \\
Y(0)=0
\end{gathered}
$$

\begin{tabular}{|c|c|c|c|c|}
\hline Symbol & Description & $\begin{array}{l}\text { Value for } \\
\text { Normal } \\
\text { Patient }\end{array}$ & $\begin{array}{l}\text { Value for } \\
\text { Patient } \\
\text { inflicted } \\
\text { with } \\
\text { T2DM }\end{array}$ & Units \\
\hline$V_{G}$ & $\begin{array}{l}\text { Distribution volume of } \\
\text { glucose }\end{array}$ & 1.88 & 1.49 & $d l / k g$ \\
\hline$k_{1}$ & & 0.065 & 0.042 & $\min ^{-1}$ \\
\hline$k_{2}$ & Rate constant & 0.079 & 0.071 & $\min ^{-1}$ \\
\hline$V_{I}$ & $\begin{array}{l}\text { Distribution volume of } \\
\text { insulin }\end{array}$ & 0.05 & 0.04 & $l / k g$ \\
\hline
\end{tabular}

The parameter values used for the Dalla Man meal model is given below.

Table 1: Parameters used for the mixed meal model 


\begin{tabular}{|c|c|c|c|c|}
\hline Symbol & Description & $\begin{array}{l}\text { Value for } \\
\text { Normal } \\
\text { Patient }\end{array}$ & $\begin{array}{l}\text { Value for } \\
\text { Patient } \\
\text { inflicted } \\
\text { with } \\
\text { T2DM }\end{array}$ & Units \\
\hline$m_{1}$ & $\begin{array}{l}\text { Rate of hepatic clear- } \\
\text { ance }\end{array}$ & 0.190 & 0.379 & $\min ^{-1}$ \\
\hline$m_{2}$ & Rate constant & 0.484 & 0.673 & $\min ^{-1}$ \\
\hline$m_{4}$ & $\begin{array}{l}\text { Rate of peripheral } \\
\text { degradation }\end{array}$ & 0.194 & 0.269 & $\min ^{-1}$ \\
\hline$m_{5}$ & Rate constant & 0.0304 & 0.0526 & $\min \cdot \mathrm{kg} / \mathrm{pmol}$ \\
\hline$m_{6}$ & Rate constant & 0.6471 & 0.8118 & \\
\hline$H E_{b}$ & $\begin{array}{l}\text { Hepatic Extraction of in- } \\
\text { sulin at basal state }\end{array}$ & 0.6 & 0.6 & \\
\hline$k_{\max }$ & & 0.0558 & 0.0465 & $m i n^{-1}$ \\
\hline$k_{m i n}$ & & 0.0080 & 0.0076 & $\min ^{-1}$ \\
\hline$k_{a b s}$ & $\begin{array}{l}\text { Intestinal absorption } \\
\text { rate }\end{array}$ & 0.0057 & 0.023 & $\min ^{-1}$ \\
\hline$k_{g r i}$ & Grinding rate & 0.0558 & 0.0465 & $m i n^{-1}$ \\
\hline$f$ & $\begin{array}{l}\text { Fraction of intestinal ab- } \\
\text { sorption that actually }\end{array}$ & 0.90 & 0.90 & \\
\hline
\end{tabular}




\begin{tabular}{|c|c|c|c|c|}
\hline Symbol & Description & $\begin{array}{l}\text { Value for } \\
\text { Normal } \\
\text { Patient }\end{array}$ & $\begin{array}{l}\text { Value for } \\
\text { Patient } \\
\text { inflicted } \\
\text { with } \\
\text { T2DM }\end{array}$ & Units \\
\hline$a$ & Rate constant & 0.00013 & 0.00006 & $m g^{-1}$ \\
\hline$b$ & Rate constant & 0.82 & 0.68 & \\
\hline$c$ & Rate constant & 0.00236 & 0.00023 & $m g^{-1}$ \\
\hline$d$ & Rate constant & 0.010 & 0.09 & \\
\hline$k_{p 1}$ & $\begin{array}{l}\text { Extrapolated EGP at } \\
\text { zero glucose and insulin }\end{array}$ & 2.7 & 3.09 & $\mathrm{mg} / \mathrm{kg} / \min$ \\
\hline$k_{p 2}$ & $\begin{array}{l}\text { Liver glucose effective- } \\
\text { ness }\end{array}$ & 0.0021 & 0.0007 & $\min ^{-1}$ \\
\hline$k_{p 3}$ & $\begin{array}{l}\text { Amplitude of insulin ac- } \\
\text { tion on the liver }\end{array}$ & 0.009 & 0.005 & $\begin{array}{l}\mathrm{mg} / \mathrm{kg} / \mathrm{min} \\
\text { per } \mathrm{pmol} / \mathrm{l}\end{array}$ \\
\hline$k_{p 4}$ & $\begin{array}{l}\text { Amplitude of portal in- } \\
\text { sulin action on the liver }\end{array}$ & 0.0618 & 0.0786 & $\begin{array}{l}\mathrm{mg} / \mathrm{kg} / \mathrm{min} \\
\text { per } \mathrm{pmol} / \mathrm{kg}\end{array}$ \\
\hline$k_{i}$ & $\begin{array}{l}\text { Delay between insulin } \\
\text { signal and insulin action }\end{array}$ & 0.0079 & 0.0066 & $\min ^{-1}$ \\
\hline$F_{c n s}$ & $\begin{array}{l}\text { Glucose uptake by the } \\
\text { brain and erythrocytes }\end{array}$ & 1.0 & 1.0 & $\mathrm{mg} / \mathrm{kg} / \mathrm{min}$ \\
\hline$V_{m 0}$ & & 2.5 & 4.65 & $\mathrm{mg} / \mathrm{kg} / \mathrm{min}$ \\
\hline
\end{tabular}




\begin{tabular}{|c|c|c|c|c|}
\hline Symbol & Description & $\begin{array}{l}\text { Value for } \\
\text { Normal } \\
\text { Patient }\end{array}$ & $\begin{array}{l}\text { Value for } \\
\text { Patient } \\
\text { inflicted } \\
\text { with } \\
\text { T2DM }\end{array}$ & Units \\
\hline$V_{m x}$ & & 0.047 & 0.034 & $\begin{array}{l}\mathrm{mg} / \mathrm{kg} / \mathrm{min} \\
\text { per } \mathrm{pmol} / \mathrm{l}\end{array}$ \\
\hline$K_{m 0}$ & & 225.59 & 466.21 & $m g / k g$ \\
\hline$p_{2 U}$ & $\begin{array}{l}\text { Insulin action on the pe- } \\
\text { ripheral glucose utiliza- } \\
\text { tion }\end{array}$ & 0.0331 & 0.0840 & $m i n^{-1}$ \\
\hline$K$ & & 2.30 & 0.99 & $\begin{array}{l}\text { pmol/kg per } \\
m g / d l\end{array}$ \\
\hline$\alpha$ & $\begin{array}{l}\text { Delay between glucose } \\
\text { signal and insulin secre- } \\
\text { tion }\end{array}$ & 0.050 & 0.0 .13 & $\min ^{-1}$ \\
\hline$\beta$ & $\begin{array}{l}\text { Pancreatic responsivity } \\
\text { to glucose }\end{array}$ & 0.11 & 0.05 & $\begin{array}{l}\mathrm{pmol} / \mathrm{kg} / \mathrm{min} \\
\text { per } \mathrm{mg} / \mathrm{dl}\end{array}$ \\
\hline$\gamma$ & & 0.5 & 0.5 & $\min ^{-1}$ \\
\hline$k_{e 1}$ & $\begin{array}{l}\text { Glomerular filtration } \\
\text { rate }\end{array}$ & 0.0 .0005 & 0.0007 & $\min ^{-1}$ \\
\hline$k_{e 2}$ & $\begin{array}{l}\text { Renal threshold of glu- } \\
\text { cose }\end{array}$ & 339 & 269 & $m g / k g$ \\
\hline
\end{tabular}




\subsection{Control Algorithm}

This section introduces the idea behind Model Predictive Control (MPC). MPC has been used widely in literature for many years. In principle, the past data is used to estimate the parameters that are part of the model. The objective of the controller is then to try and predict the behaviour of the system based on the given parameters and/or equations that are part of the model. In addition, it should be noted that most MPC are designed such that the output, $x(t)$, is as close to a set point as possible. For example, with respect to the glucose levels in a given persons blood stream, the ideal case is to keep the glucose concentration as close to $5 \mathrm{mmol} / \mathrm{L}$ as possible. For the purposes of simplicity, the feedback controller was replaced with a feed-forward controller. A feed-forward controller can assess disturbances (i.e. meals being processed by the human body) but does not have any feedback on the output (i.e. the glucose concentration of the human body). The model chosen for the MPC is a Hovorka model which is described in [103]. This section will be broken down into two sections: the first will describe the model in detail; and, the second will describe the linear application of the MPC around the steady state.

\subsubsection{MPC Model Simulation}

The Hovorka model can be broken down into three components: The glucose absorption; the insulin absorption; and, the glucose regulatory system. It consists of two inputs and two outputs. The inputs are meal disturbances and insulin infusions, whereas the outputs are glucose and insulin concentrations in the blood stream. For ease of understanding, a similar approach to the meal model simulation description will be applied to this section. 


\subsubsection{The glucose absorption}

As its name suggests, this particular component is responsible for processing the

effects of meals on the glucose rate. It is described by three linear equations as follows:

$$
\begin{aligned}
\frac{d D_{1}}{d t}(t) & =A_{G} d(t)-\frac{D_{1}(t)}{\tau_{D}} \\
\frac{d D_{2}}{d t}(t) & =\frac{D_{1}(t)}{\tau_{D}}-\frac{D_{2}(t)}{\tau_{D}} \\
U_{G}(t) & =\frac{D_{2}(t)}{\tau_{D}}
\end{aligned}
$$

Where:

$A_{G}$ refers to the carbohydrate bio-availability parameter (this is a constant value of 0.8 , the rest, 0.2 of the carbohydrates are lost in processing); $d(t)$ refers to amount of carbohydrates in the meal (in $\mathrm{CHO} / \mathrm{min}$ );

$\tau_{D}$ refers to the time constant for glucose absorption (this is constant value of 40 minutes); and, $U_{G}$ refers to the glucose absorption rate (in $\mathrm{mmol} / \mathrm{min}$ )

\subsubsection{The insulin absorption}

Very similar to the glucose absorption, the insulin absorption component is responsible for processing the effects of insulin in the body. It is also described by three linear 
equations as follows:

$$
\begin{aligned}
\frac{d S_{1}}{d t}(t) & =u(t)-\frac{S_{1}(t)}{\tau_{I}} \\
\frac{d S_{2}}{d t}(t) & =\frac{S_{1}(t)}{\tau_{I}}-\frac{S_{2}(t)}{\tau_{I}} \\
U_{I} & =\frac{S_{2}(t)}{\tau_{I}}
\end{aligned}
$$

Where:

$u(t)$ refers to the insulin injected (in $\mathrm{mU} / \mathrm{min})$;

$\tau_{I}$ refers to the time constant for insulin absorption (this is constant value of 55 minutes); and,

$U_{I}$ refers to the insulin rate which enters the blood stream (in $\mathrm{mU} / \mathrm{min}$ ).

\subsubsection{The glucose regulatory system}

The glucose regulatory system is the most complicated subsystem in the MPC model. There are two inputs the glucose and the insulin appearance rate in the blood stream. There are two main processes that are interconnected in this compartment: glucose processing and insulin processing. The first, glucose processing starts once the glucose has been absorbed from the meal by the body. It then moves to one of two subcompartments: $Q_{1}(t)$ or $Q_{2}(t)$. The first sub-compartment, $Q_{1}(t)$ represents the amount of glucose within the bloodstream; whereas, the second, $Q_{2}(t)$ represents the amount of glucose in the muscles and tissues. The system is described by the following 
set of linear equations:

$$
\begin{aligned}
\frac{d Q_{1}}{d t}(t) & =U_{G}(t)-F_{01, c}(t)-F_{R}(t)-x_{1} Q_{1}(t)+k_{12} Q_{2}(t)+E G P_{0}\left(1-x_{3}(t)\right) \\
\frac{d Q_{2}}{d t}(t) & =x_{1}(t) Q_{1}(t)-k_{12} Q_{2}(t)-x_{2}(t) Q_{2}(t) \\
G(t) & =\frac{Q-1(t)}{V_{G}}
\end{aligned}
$$

Where:

$F_{01, c}(t)$ refers to the glucose utilisation by the central nervous system (in $\mathrm{mmol} / \mathrm{min}$ ); $F_{R}(t)$ refers to the glucose excreted by the body, namely the kidneys (in $\mathrm{mmol} / \mathrm{min}$ ); $E G P_{0}$ refers to the amount of glucose produced by the liver when the insulin level is zero $(\mathrm{mmol} / \mathrm{min})$;

$k_{12}$ refers to the transfer rate of glucose from the bloodstream to the muscles and tissues;

$x_{1}(t)$ refers to the state in which the insulin interaction influences the glucose transport and distribution;

$x_{3}$ refers to the in which the insulin interaction influences the endogenous production of glucose in the liver; and, $V_{G}$ refers to the glucose distribution volume and is dependent on the body weight; $G(t)$ refers to the glucose concentration in the blood $(\mathrm{mmol} / \mathrm{L})$.

The glucose utilisation by the central nervous system is highly dependent on the glucose consumption that is independent of insulin. This is described by the following equation: 


$$
F_{01, c}= \begin{cases}F_{01} & \text { if } G(t) \leq 4.5 \\ \frac{F_{01} G(t)}{4.5} & \text { if } G(t)<4.5\end{cases}
$$

The glucose excreted by the kidneys is defined by the following equation:

$$
F_{R}= \begin{cases}0.003(G(t)-9) V_{G} & \text { if } G(t) \geq 9 \\ 0 & \text { if } G(t)<9\end{cases}
$$

There are three main states that are involved with the insulin interaction in the glucose regulatory system: $x_{1}(t), x_{2}(t)$; and, $x_{3}(t)$. These states are dependent only on the plasma insulin concentration and are influenced by the glucose transport and distribution, glucose disposal, and endogenous production of glucose in the liver. The equations that describe the insulin interactions in the glucose regulatory component are as follows:

$$
\begin{aligned}
\frac{d x_{1}}{d t}(t) & =-k_{a 1} x_{1}(t)+k_{b 1} I(t) \\
\frac{d x_{2}}{d t}(t) & =-k_{a 2} x_{1}(t)+k_{b 2} I(t) \\
\frac{d x_{3}}{d t}(t) & =-k_{a 3} x_{1}(t)+k_{b 3} I(t) \\
\frac{d I}{d t}(t) & =\frac{U_{I}(t)}{V_{I}}-k_{e} I(t)
\end{aligned}
$$

Where:

$F_{01, c}(t)$ refers to the glucose utilisation by the central nervous system (in $\mathrm{mmol} / \mathrm{min}$ ); 
$F_{R}(t)$ refers to the glucose excreted by the body, namely the kidneys (in $\mathrm{mmol} / \mathrm{min}$ ); $E G P_{0}$ refers to the amount of glucose produced by the liver when the insulin level is zero $(\mathrm{mmol} / \mathrm{min})$;

$x_{12}$ refers to the transfer rate of glucose from the bloodstream to the muscles and tissues;

$k_{a 1} ; k_{a 2} ;$ and,$k_{a 3}$ refers to the deactivation rates which were experimentally estimated; $k_{b 1} ; k_{b 2} ;$ and, $k_{b 3}$ refers to the activation rates which are calculated from the corresponding insulin sensitivities (see equations 34-36); and,

$V_{I}$ refers to the insulin distribution volume.

The activation rates are calculated based on their corresponding insulin sensitives:

- Insulin transportation and distribution sensitivity $\left(S_{I, 1}\right)$

- Insulin disposal sensitivity $\left(S_{I, 2}\right)$

- Insulin endogenous production sensitivity $\left(S_{I, 3}\right)$

It should be noted that these sensitivities will vary from patient to patient and will be dependent on what activity or stress level the patient is enduring. Thus, these sensitivities may need to reanalysed and computed from time to time during a routine doctor's visit.

$$
\begin{aligned}
k_{b 1} & =S_{I T} \cdot k_{a 1} \\
& =3.07 \times 10^{-5} \mathrm{~L} / \mathrm{min} / \mathrm{mU} \\
k_{b 2} & =S_{I D} \cdot k_{a 2} \\
& =4.92 \times 10^{-5} \mathrm{~L} / \mathrm{min} / \mathrm{mU}
\end{aligned}
$$




$$
\begin{aligned}
k_{b 2} & =S_{I E} \cdot k_{a 3} \\
& =1.6 \times 10^{-3} \mathrm{~L} / \mathrm{min} / \mathrm{mU}
\end{aligned}
$$

Lastly, like the meal model simulation, the following table is a summary of all the constants used in the MPC.

\begin{tabular}{|c|c|c|c|}
\hline Symbol & Description & Value & Units \\
\hline$k_{12}$ & $\begin{array}{l}\text { Transfer rate of glucose from the blood- } \\
\text { stream to the muscles and tissues }\end{array}$ & 0.066 & $\min ^{-1}$ \\
\hline$k_{a 1}$ & $\begin{array}{l}\text { Deactivation rate of glucose distribu- } \\
\text { tion and transportation }\end{array}$ & 0.006 & $\min ^{-1}$ \\
\hline$k_{a 2}$ & Deactivation rate of glucose disposal & 0.06 & $\min ^{-1}$ \\
\hline$k_{a 3}$ & $\begin{array}{l}\text { Deactivation rate of endogenous pro- } \\
\text { duction of glucose }\end{array}$ & 0.03 & $\min ^{-1}$ \\
\hline$k_{b 1}$ & $\begin{array}{l}\text { Activation rate of glucose transporta- } \\
\text { tion and distribution }\end{array}$ & $3.07 \times 10^{-5}$ & $\mathrm{~L} / \mathrm{min} / \mathrm{mU}$ \\
\hline$k_{b 2}$ & Activation rate of glucose disposal & $4.92 \times 10^{-5}$ & $\mathrm{~L} / \mathrm{min} / \mathrm{mU}$ \\
\hline$k_{b 3}$ & $\begin{array}{l}\text { Activation rate of endogenous produc- } \\
\text { tion of glucose }\end{array}$ & $1.6 \times 10^{-3}$ & $\mathrm{~L} / \mathrm{min} / \mathrm{mU}$ \\
\hline$k_{e}$ & Insulin elimination rate & 0.138 & $\min ^{-1}$ \\
\hline
\end{tabular}

Table 2: Parameters used for MPC model 


\begin{tabular}{|c|c|c|c|}
\hline Symbol & Description & Value & Units \\
\hline$\tau_{D}$ & Carbohydrate absorption rate & 40 & $\min$ \\
\hline$\tau_{I}$ & Insulin absorption & 55 & $\min$ \\
\hline$A_{G}$ & Carbohydrate utilisation & 0.8 & - \\
\hline$S_{I, 1}$ & $\begin{array}{l}\text { Insulin transportation and distribution } \\
\text { sensitivity }\end{array}$ & $51.2 \times 10^{-2}$ & $\mathrm{~L} / \mathrm{mU}$ \\
\hline$S_{I, 2}$ & Insulin disposal sensitivity & $8.2 \times 10^{-4}$ & $\mathrm{~L} / \mathrm{mU}$ \\
\hline$S_{I, 3}$ & $\begin{array}{l}\text { Insulin endogenous production sensi- } \\
\text { tivity }\end{array}$ & $8.2 \times 10^{-4}$ & $\mathrm{~L} / \mathrm{mU}$ \\
\hline$\frac{V_{I}}{B W}$ & Insulin distribution volume & 0.12 & $\mathrm{~L} / \mathrm{kg}$ \\
\hline$\frac{V_{G}}{B W}$ & Glucose distribution volume & 0.16 & $\mathrm{~L} / \mathrm{kg}$ \\
\hline$\frac{E G P_{0}}{B w}$ & Liver glucose production & 0.0161 & $\mathrm{mmol} / \mathrm{min} / \mathrm{kg}$ \\
\hline$\frac{F_{01}}{B W}$ & $\begin{array}{l}\text { Glucose utilisation by the central ner- } \\
\text { vous system }\end{array}$ & 0.0097 & $\mathrm{mmol} / \mathrm{min} / \mathrm{kg}$ \\
\hline
\end{tabular}

For the purposes of this document, the Hovorka model was used only as a basis for the MPC because the model described above is a non-linear approximation. However, the MPC that this document would like to explore is that of a linear model. The linear approximation in MPC is used when calculating predictions because it is faster and much more simpler in comparison to the non-linear model. The timing of the predictions is time sensitive due to the short time intervals. Lastly, a linearised adaptation of the model also aids in robust optimisation problems in comparison to 
a non-liner model which exponentially increases its chance of diverging.

In a normal individual, the blood glucose levels are between 4-6 mmol/L while fasting; or $4-8 \mathrm{mmol} / \mathrm{L}$ after consumption of a meal [3]. Due to these values, the set point for blood glucose concentration would ideally be $5 \mathrm{mmol} / \mathrm{L}$. This in turn would be in the mid range of both fasting and non-fasting blood glucose levels. A linearized version of the Hovorka was developed by [104]. The steady states for all variables were computed when the glucose concentrations were equal to $5 \mathrm{mmol} / \mathrm{L}$. This resulted in a basal insulin injection of $6.677 \mathrm{mU} / \mathrm{min}$ if the meal was ingested at steady state. 


\section{Chapter 4}

\section{Simulation Results and Discussion}

As its name suggests, this section of the thesis presents the simulation results obtained from the models described in Chapter 3 and then discusses them based on results from literature. There are two models that are under consideration here: first the meal model simulation; and, the MPC. The meal model was created from existing equations derived from the Dalla Man model. As expected, the simulation resulted in results that were quite comparable to that obtained from the Dalla Man model itself (see Figure 9 - 14). It displays one of the advantage of hierarchical modelling examination of dynamic interactions between a single level and between its sub-levels.

However, there are major drawbacks in the Dalla Man model. This includes but is not limited to: replacement of models with more up-to-date mechanistic models; including a liver glucose utilisation and other hormones. First, the replacement of more up-todate models. Although the Dalla Man model is considered the gold standard, there are many sub-models today that are presented in literature. These models act in isolation to capture the interaction of one key component in the complex glucose and insulin subsystem. A prime example of this would be the $\beta$-cells model. The current representation of the $\beta$-cells in the pancreas in the Dalla Man model appears to be in its infancy with respect to the details present. Yes, it represents the whole body 
model. However, it does not define the behaviour of the system down to the cell level ${ }^{1}$. Considering that the model presented above was modelled in hierarchical structure, replacing certain sub-components as newer research has been conducted will not pose a problem. Secondly, the liver's glucose uptake is not considered in the development of the model. This leads to believe that the other components are compensating for this (i.e. muscle and adipose tissue). Another drawback is the misrepresentation of feedback signals from the muscle and adipose tissue model to the rest of the body. Lastly the model only includes meal digestion and two key hormones - glucose and insulin. However, in reality, the muscle and adipose tissue send out signals for the uptake of various hormones that may hinder the uptake of glucose and/or insulin. A prime example of this is glucagon. The problem with the exclusion of key hormones is that it would require structural changes to the model itself. Nevertheless, if one would like to develop a model that simulates both low level and high level interactions.

The MPC model, that was derived from the Hovorka model however did not perform well in comparison to the Dalla Man model. The bigger the meal the further disparity in the set-point glucose concentration. But, this is explainable since the bigger the meal ingested, the further away the glucose level would be from steady state which would mean that the assumption (meal ingested at steady state) when linearising the model is incorrect. Thus in turn the results do not quite match up with the body's absorption. Another observation is that, there are times that the model looses control over the glucose concentrations. This indicates that a linearised controller would have no control if the patient was to go into the hypoglycemic stage (low blood sugar).

Like the Dalla Man model, there are also drawbacks to the MPC model (see Figure 15). One major problem is that the human body does not react in a linear fashion.

\footnotetext{
${ }^{1}$ A cell level model would be ideal to test not only algorithms designed for validating DM but better understanding other disease and finding cures for them. In addition having cell level model will aid in designing a better CAD as the insulin glucose interactions will be better understood.
} 
Thus, modelling an artificial device in such a way will not yield desirable results. Having a non-linear model would better aid in maintaining the blood glucose level between 4 and $8 \mathrm{mmol} / \mathrm{L}$ for meals that contain a high level of glucose and/or carbohydrate content. However, like in the linear model, the meals would have to be ingested at predetermined times in order for the model to work ideally ${ }^{2}$. The compromise to this approach would be the computational time. While the non-linear model would give results that are more accurate to the Hovorka model, it would need a higher calculation capacity and thus requiring more time. Which in turn would mean that one could not have a sampling size that is small enough for the safety of the patient so that he/she does no go into hypoglycemic and/or hyperglycemic conditions.

\footnotetext{
${ }^{2}$ The model could work just as effectively without predetermined meal times and within the range of normal human behaviour; but, the results would not be ideal.
} 


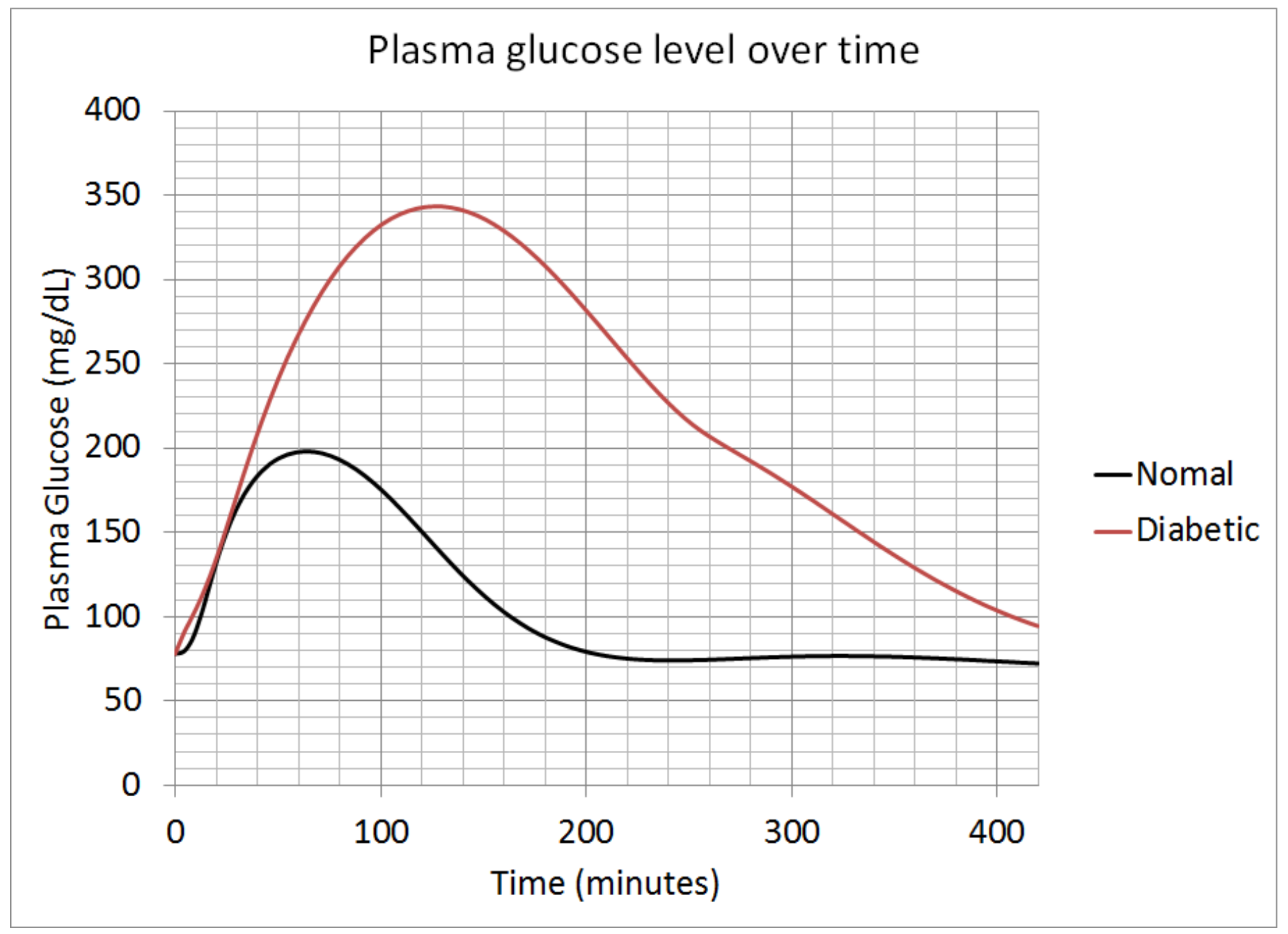

Figure 9: Simulation of plasma glucose level of a non-diabetic patient weighing $78.0 \mathrm{~kg}$ graphed in excel 


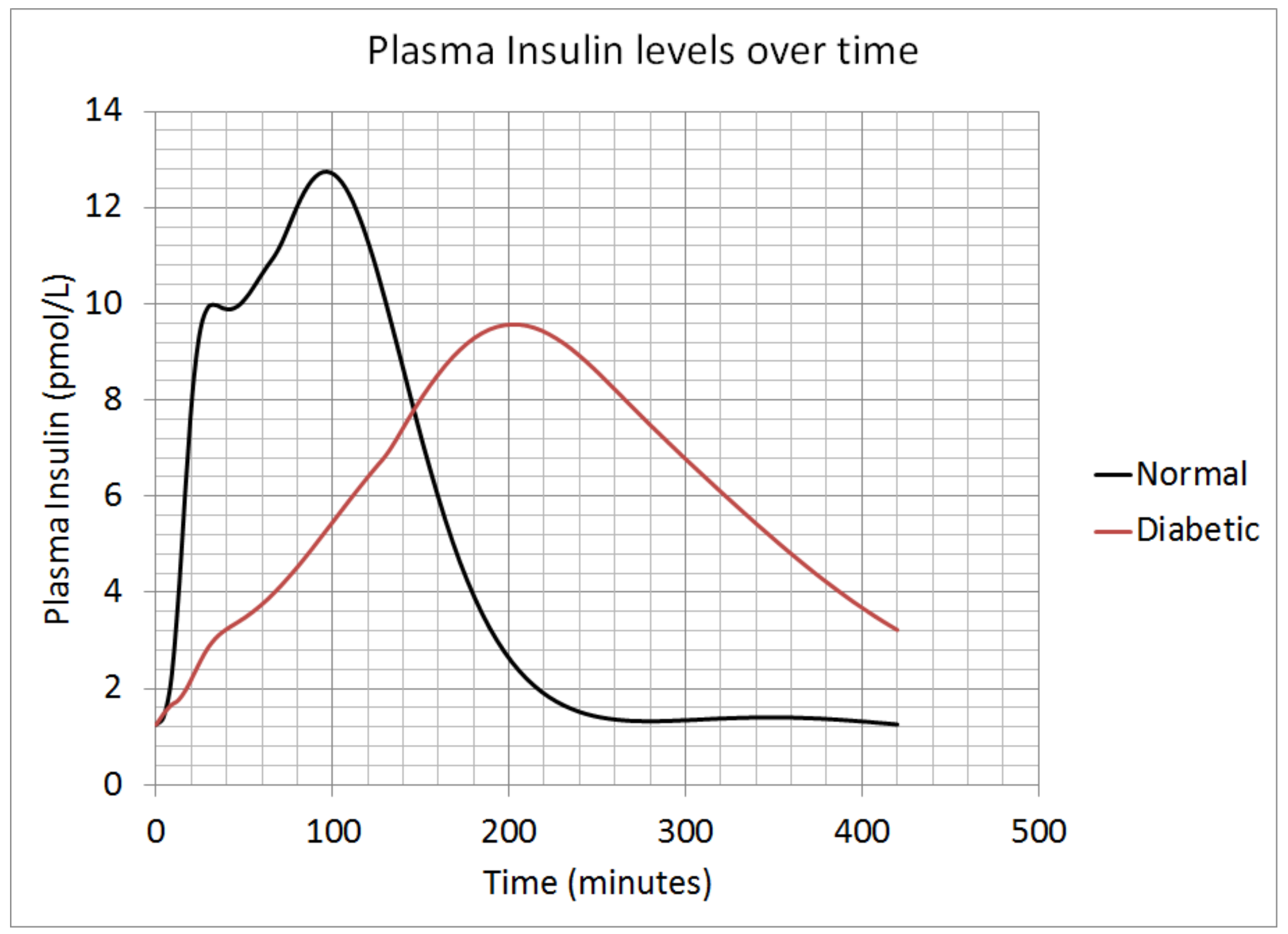

Figure 10: Simulation of plasma insulin level of a non-diabetic patient weighing $78.0 \mathrm{~kg}$ graphed in excel 


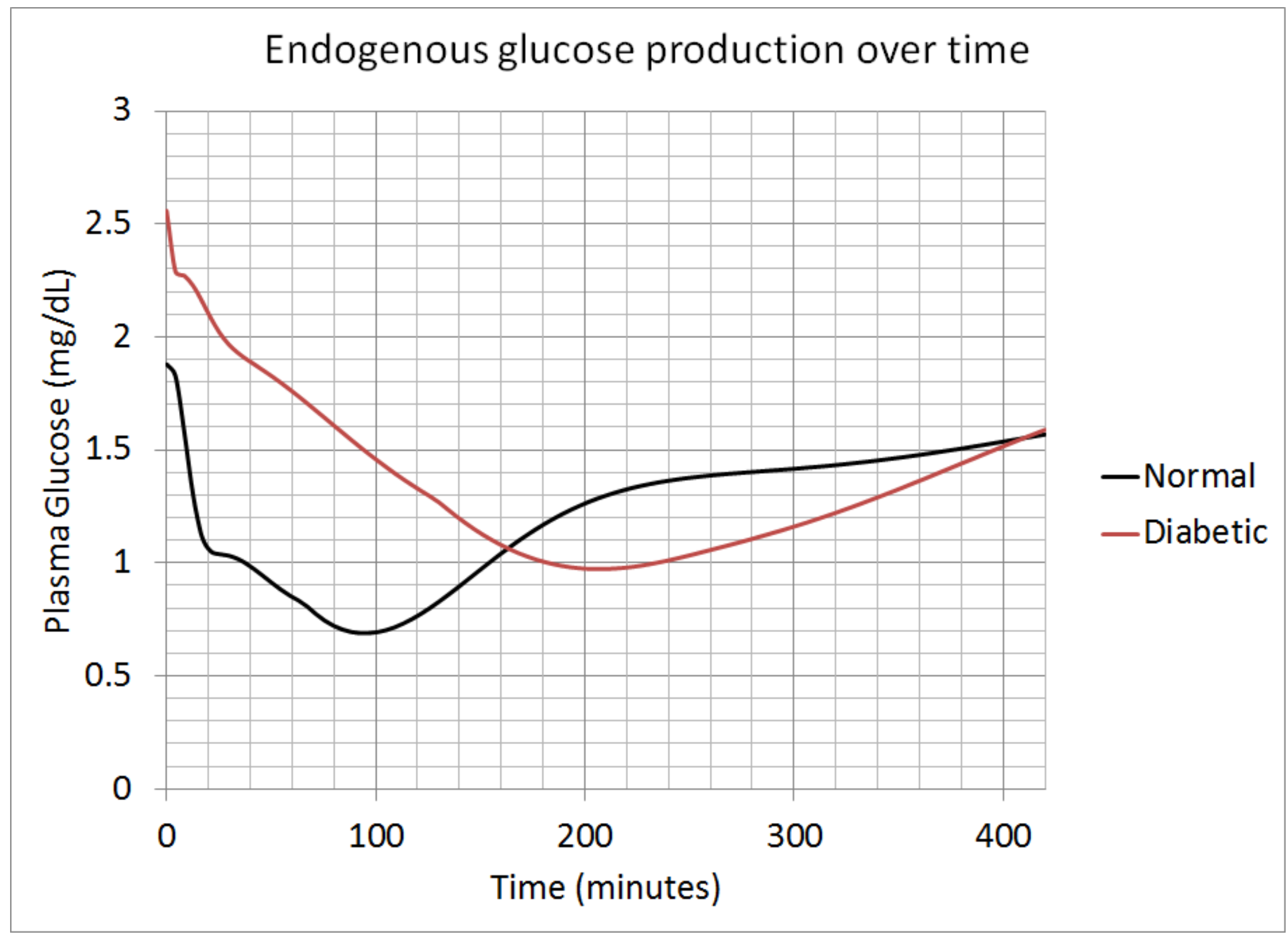

Figure 11: Simulation of Endogenous Glucose Production level of a non-diabetic patient weighing $78.0 \mathrm{~kg}$ graphed in excel 


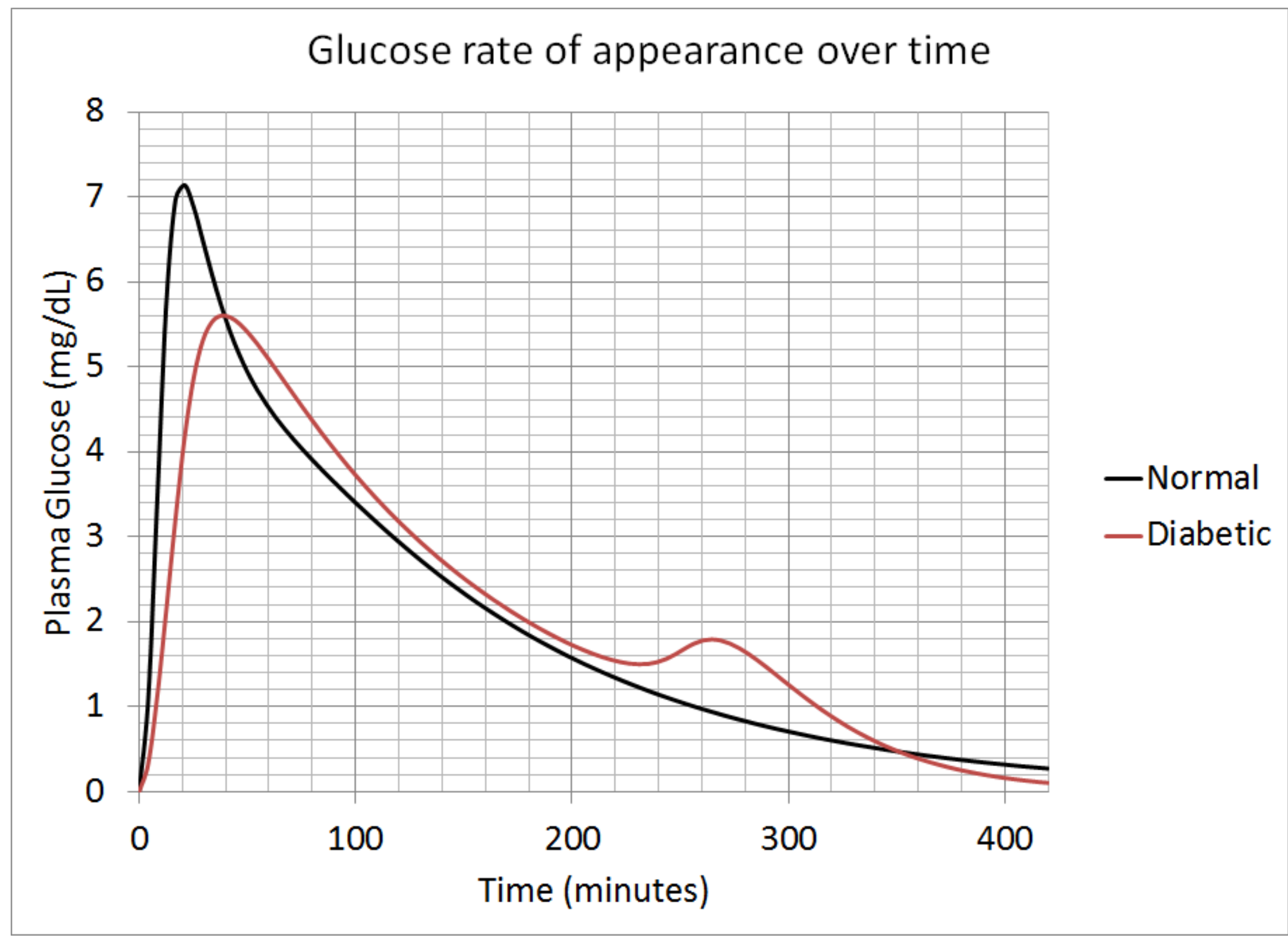

Figure 12: Simulation of Endogenous Glucose Rate Of Appearance of a non-diabetic patient weighing 78.0 kg graphed 


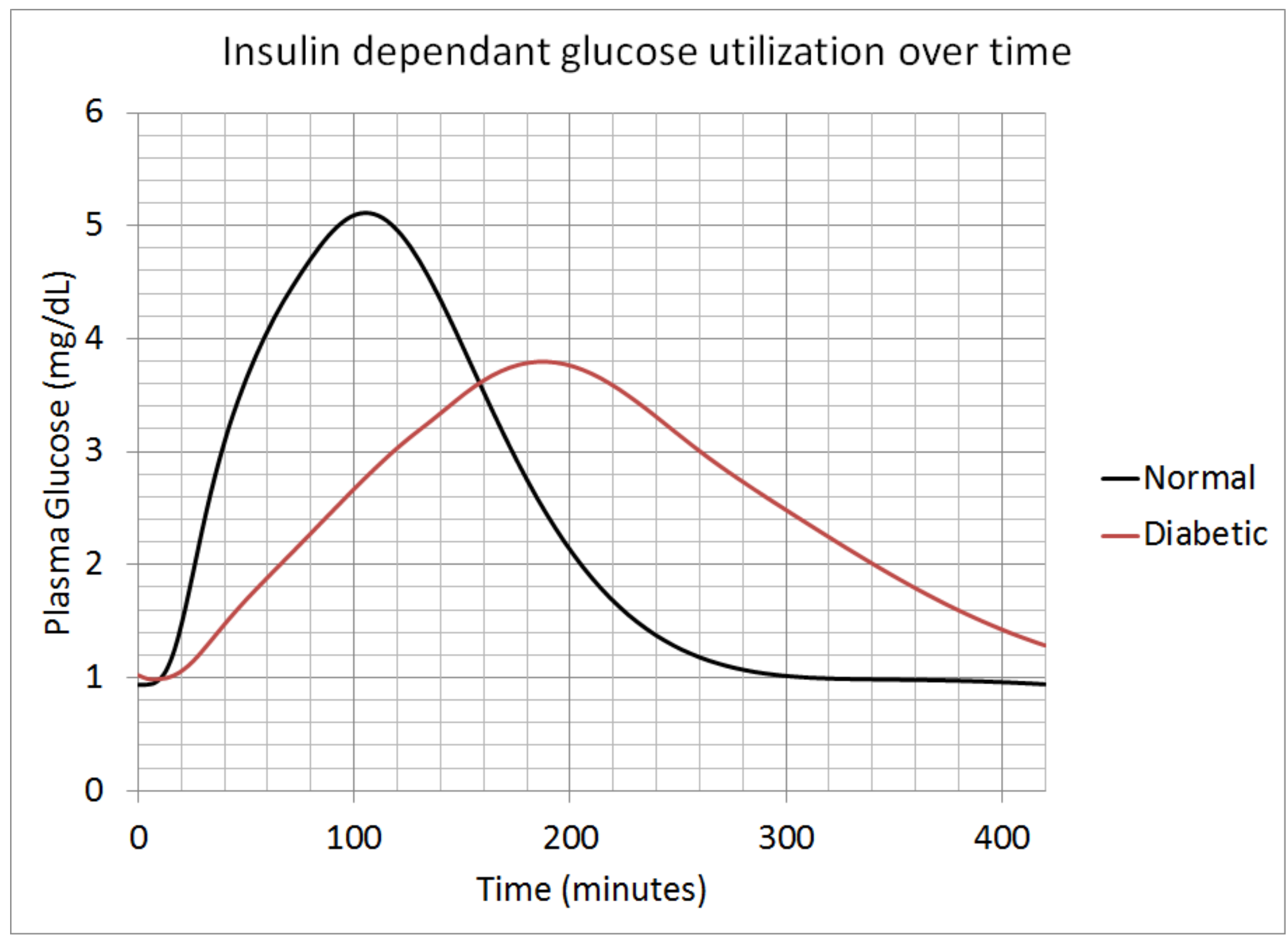

Figure 13: Simulation of insulin dependent Glucose Utilization of a non-diabetic patient weighing $78.0 \mathrm{~kg}$ graphed in excel 


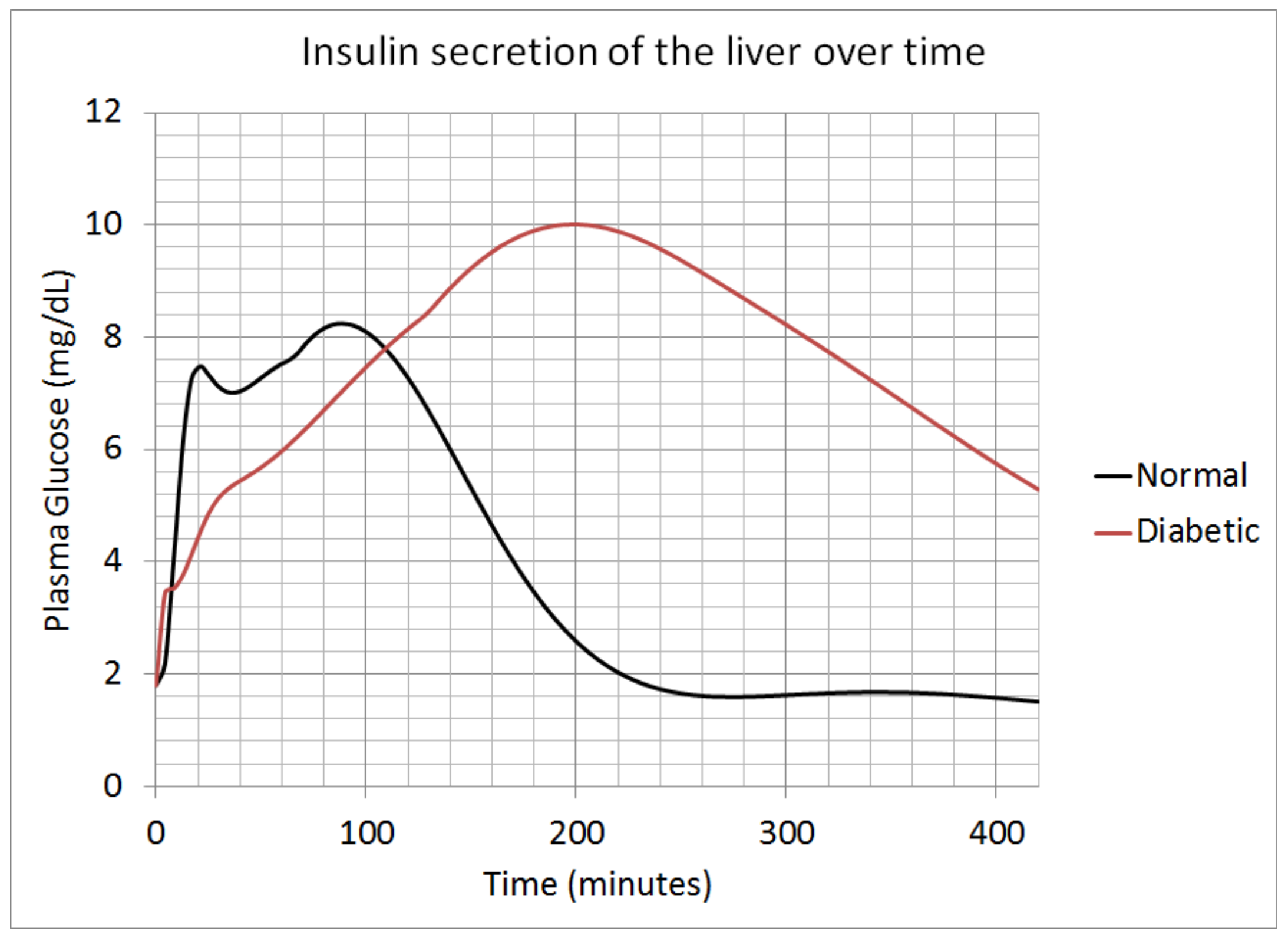

Figure 14: Simulation of Insulin secreted by the liver of a non-diabetic patient weighing $78.0 \mathrm{~kg}$ graphed in excel 

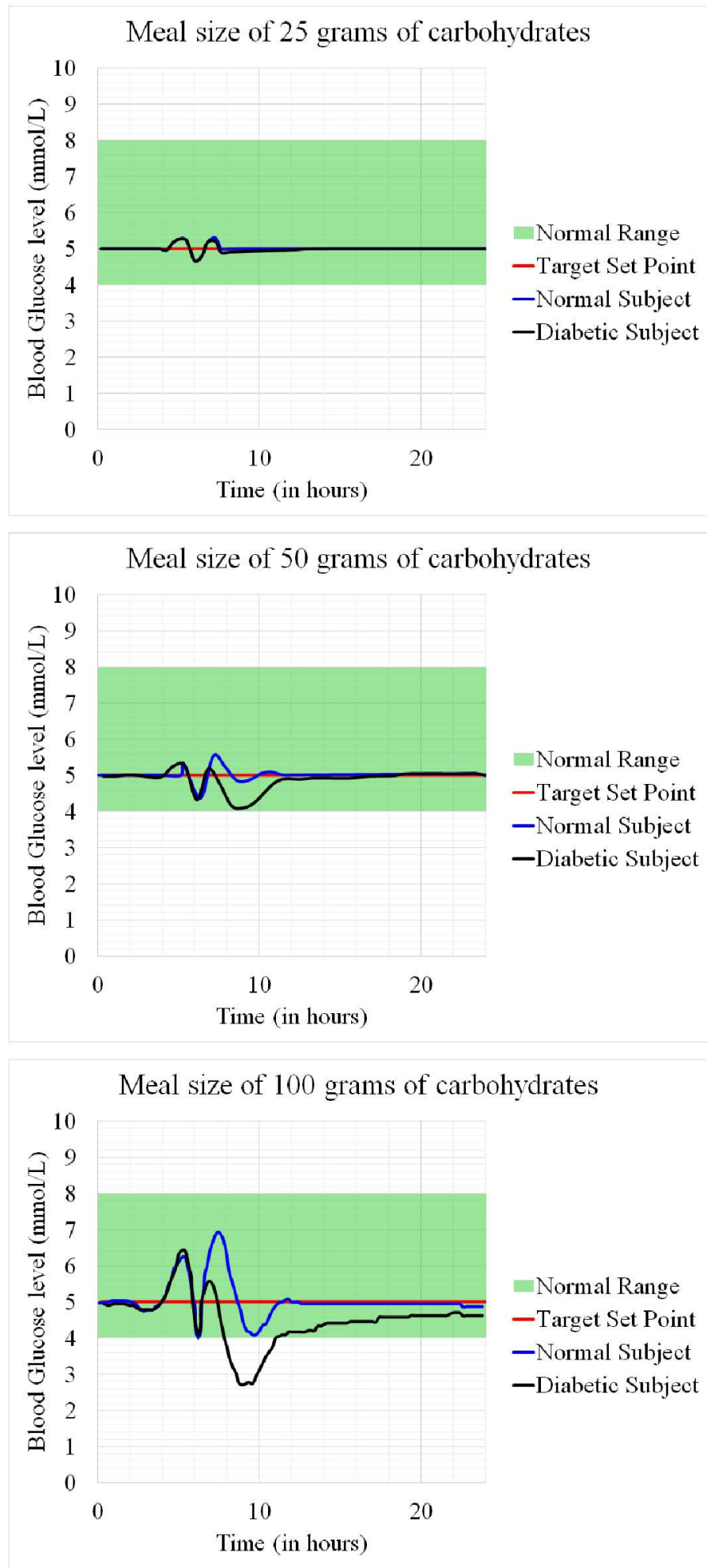

Figure 15: Simulation results of MPC based on a linearised model of the Hovorka model. 


\section{Chapter 5}

\section{Conclusion}

DM is a disease that involves a complex glucose regulatory system which involves different levels of the human body system. It involves systems from the cellular level which govern the absorption, production and intake of substances (for the purposes of this document - glucose and insulin). But, at the same time it involves the whole body itself, where substances (i.e. glucose and insulin) are transported between organs in the body. In order to fully understand this complex behaviour, one has to simulate the system combining all different levels and thus in turn understanding its full complexity. This has been achieved easily by DEVS in a hierarchical form. The first model, which is the physiological model examines the glucose insulin interactions in the human body. The results are comparable to literature and can be easily modified to include other elements of this complex system such as glucose utilisation of the liver and other hormones.

The second model, is a linearised version of the Hovorka model. It proves that a linear version would greatly underestimate the effect insulin has on the body. However, this was expected because the model was based on linear approximations. Which indicates that any prediction that are farthest into the future will have the most error. A non-linear model will be computationally heavy and thus requires more time 
to compute which could potentially compromise the safety of the patient. However, given the computational power and resources now available, this should not be an issue. Lastly, an adaptive controller would ensure that the parameters such as insulin sensitives would adapt as the body changes everyday.

In order to implement this DEVS model into a a real time system on a microcontroller. Once just has to connect the glucose sensor and insulin infusion pumps to the micro-controller, download the project and simulate it in DEVSRT; which in turn would result in a real time simulation of the above model.

\subsection{Future Work}

In addition to the enhancements stated above, one of the obvious steps to enhance this thesis would be to write a driver that would particularly read actual data from a FDA approved continuous glucose monitor and write the data to another FDA approved device, the insulin pump. The model itself can be used to compute the right amounts of insulin that the patient needs to be injected given the reading from the glucose monitor. Furthermore, the system itself could be connected to a secure connection that would alert authorities if the patient's life was in danger. While this is a solvable problem one should note that it could potentially bring up other problems such as patient confidentiality.

This system could also be enhanced to include some/all of the growing common forms of diabetes; especially those that have strong links to other disorders and/or diseases. This includes the newly discovered Type 3 diabetes; which in turn would help find a cure for Alzheimer's Disease (AD) [105] which is described in Annex A.1 as Type 3 DM. However at this time, more studies and research need to be conducted in order to be able to develop a model and thus simulate it. 


\section{References}

[1] F. J. Doyle III, B. W. Bequette, R. Middleton, B. Ogunnaike, B. Paden, R. S. Parker, and M. Vidyasagar, "Control in biological systems," The Impact of Control Technology, 2011.

[2] G. A. Wainer, Discrete-Event Modeling and Simulation: A Practitioner's Approach, 1st ed. Boca Raton, FL, USA: CRC Press, Inc., 2009.

[3] I. D. Federation, IDF Diabetes Atlas, 7th ed. International Diabetes Federation, 2015.

[4] van der Sangen Kim, "Artificial pancreas - "next innovation" in diabetes care - health tech event," Online, Dec 2014.

[5] Juvenile Diabetes Research Foundation, "Artificial pancreas project research," Online, Dec 2014.

[6] R. Goldstein, "Devs-based dynamic simulation of deformable biological structures," p. 207, 2009.

[7] C. Maus, M. John, M. Röhl, and A. M. Uhrmacher, "Hierarchical modeling for computational biology," in Proceedings of the Formal Methods for the Design of Computer, Communication, and Software Systems 8th International Conference on Formal Methods for Computational Systems Biology, ser. SFM'08. Berlin, Heidelberg: Springer-Verlag, 2008, pp. 81-124.

[8] H. Sauro, D. Harel, A. Uhrmacher, M. Hucka, M. Kwiatkowska, C. Shaffer, P. Mendes, L. Stromback, and J. Tyson, "Challenges for modeling and simulation methods in systems biology," in Simulation Conference, 2006. WSC 06. Proceedings of the Winter, Dec 2006, pp. 1720-1730.

[9] G. A. Wainer and P. Mosterman, Discrete-Event Modeling and Simulation: Theory and Applications. Taylor and Francis, 2011. 
[10] B. P. Zeigler, T. G. Kim, and H. Praehofer, Theory of Modeling and Simulation, 2nd ed. Orlando, FL, USA: Academic Press, Inc., 2000.

[11] J. S. van Zon and P. R. ten Wolde, "Greens-function reaction dynamics: A particle-based approach for simulating biochemical networks in time and space," The Journal of Chemical Physics, vol. 123, no. 23, pp. -, 2005.

[12] B. P. Zeigler and S. Vahie, "Devs formalism and methodology: Unity of conception/diversity of application," in In Proceedings of the 25th Winter Simulation Conference. ACM Press, 1993, pp. 573-579.

[13] J.-B. Filippi and P. Bisgambiglia, "General methodology 2: enabling large scale and high definition simulation of natural systems with vector models and jdevs." in Winter Simulation Conference, J. L. Snowdon and J. M. Charnes, Eds. ACM, 2002, pp. 1964-1970.

[14] F. J. Barros, "Abstract simulators for the dsde formalism," in Proceedings of the 30th Conference on Winter Simulation, ser. WSC '98. Los Alamitos, CA, USA: IEEE Computer Society Press, 1998, pp. 407-412.

[15] G. Wainer, "Creating advanced fire-spreading models using the cd++ toolkit," in Proceedings of the 3rd Biennial meeting of the International Environmental Modelling and Software Society (iEMSs), Burlington, VT, USA, 2006.

[16] G. Wainer, S. Jafer, B. Al-Aubidy, A. Dias, R. Bain, M. Dumontier, and J. Cheetham, "Advanced DEVS models with application to biomedicine," Artificial Intelligence, Simulation and Planning, Buenos Aires, Argentina, 2007.

[17] R. Goldstein, G. Wainer, J. Cheetham, and R. Bain, "Vesicle-synapsin interactions modeled with cell-devs," in Simulation Conference, 2008. WSC 2008. Winter, Dec 2008, pp. 813-821.

[18] A. M. Uhrmacher and C. Priami, "Discrete event systems specification in systems biology - a discussion of stochastic pi calculus and devs," in Proceedings of the 37th Conference on Winter Simulation, ser. WSC '05. Winter Simulation Conference, 2005, pp. 317-326.

[19] R. Ewald, C. Maus, A. Rolfs, and A. Uhrmacher, "Discrete event modelling and simulation in systems biology," Journal of Simulation, vol. 1, no. 2, pp. 81-96, 2007. 
[20] A. Uhrmacher, J. Himmelspach, M. Rohl, and R. Ewald, "Introducing variable ports and multi-couplings for cell biological modeling in devs," in Simulation Conference, 2006. WSC 06. Proceedings of the Winter, Dec 2006, pp. 832-840.

[21] A. Uhrmacher, R. Ewald, M. John, C. Maus, M. Jeschke, and S. Biermann, "Combining micro and macro-modeling in devs for computational biology," in Simulation Conference, 2007 Winter, Dec 2007, pp. 871-880.

[22] M. Van Schyndel, "Computational fluid dynamic solver based on cellular discrete-event simulation for use in biological systems," Master's thesis, Systems and Computer Engineering Dep. Carleton University, 1125 Colonel By Dr. Ottawa, ON, Canada K1S 5B6, 2014.

[23] A. M. Uhrmacher, J. Himmelspach, M. Jeschke, M. John, S. Leye, C. Maus, M. Rhl, and R. Ewald, "One modelling formalism \& simulator is not enough! a perspective for computational biology based on james ii." in $F M S B$, ser. Lecture Notes in Computer Science, J. Fisher, Ed., vol. 5054. Springer, 2008, pp. 123138.

[24] M. J. Byrne, M. N. Waxham, and Y. Kubota, "Cellular dynamic simulator: An event driven molecular simulation environment for cellular physiology." Neuroinformatics, vol. 8, no. 2, pp. 63-82, 2010.

[25] P. Talwar, Manual of cytogenetics in reproductive biology. New Delhi: Jaypee Brothers Medical Publishers, 2014.

[26] J. Anderson, J.D., "Basic philosophy of cfd," in Computational Fluid Dynamics, J. Wendt, Ed. Springer Berlin Heidelberg, 2009, pp. 3-14.

[27] "4 ways to get started," 2011.

[28] T. Bremer and D. A. Gough, "Is blood glucose predictable from previous values? a solicitation for data." Diabetes, vol. 48, no. 3, pp. 445-451, 1999.

[29] R. S. Parker, F. J. Doyle, and N. A. Peppas, "A model-based algorithm for blood glucose control in type i diabetic patients," Biomedical Engineering, IEEE Transactions on, vol. 46, no. 2, pp. 148-157, 1999.

[30] D. A. Finan, H. Zisser, L. Jovanovic, W. C. Bevier, and D. E. Seborg, "Identification of linear dynamic models for type 1 diabetes: a simulation study," in Proc 14th IFAC ADCHEM Symp, Gramado, Brazil, 2006. 
[31] T. Van Herpe, M. Espinoza, B. Pluymers, P. Wouters, F. De Smet, G. Van den Berghe, and B. De Moor, "Development of a critically ill patient input-output model," in System Identification, vol. 14, no. 1, 2006, pp. 481-486.

[32] S. J. Qin and T. A. Badgwell, "An overview of nonlinear model predictive control applications," in Nonlinear model predictive control. Springer, 2000, pp. 369-392.

[33] R. N. Bergman, L. S. Phillips, and C. Cobelli, "Physiologic evaluation of factors controlling glucose tolerance in man: measurement of insulin sensitivity and beta-cell glucose sensitivity from the response to intravenous glucose." Journal of Clinical Investigation, vol. 68, no. 6, p. 1456, 1981.

[34] V. W. Bolie, "Coefficients of normal blood glucose regulation," Journal of Applied Physiology, vol. 16, no. 5, pp. 783-788, 1961.

[35] E. Ackerman, L. C. Gatewood, J. W. Rosevear, and G. D. Molnar, "Model studies of blood-glucose regulation," The bulletin of mathematical biophysics, vol. 27 , no. 1, pp. 21-37, 1965.

[36] C. Cobelli, G. Federspil, G. Pacini, A. Salvan, and C. Scandellari, "An integrated mathematical model of the dynamics of blood glucose and its hormonal control," Mathematical Biosciences, vol. 58, no. 1, pp. 27 - 60, 1982.

[37] "Validation of mathematical models of complex endocrine-metabolic systems. a case study on a model of glucose regulation," Medical and Biological Engineering and Computing, vol. 21, no. 4, 1983.

[38] A. Caumo, P. Vicini, and C. Cobelli, "Is the minimal model too minimal?" Diabetologia, vol. 39, no. 8, pp. 997-1000, 1996.

[39] C. Cobelli, A. Caumo, and M. Omenetto, "Minimal model sgoverestimation and siunderestimation: improved accuracy by a bayesian two-compartment model," American Journal of Physiology-Endocrinology And Metabolism, vol. 277, no. 3, pp. E481-E488, 1999.

[40] R. Hovorka, F. Shojaee-Moradie, P. V. Carroll, L. J. Chassin, I. J. Gowrie, N. C. Jackson, R. S. Tudor, A. M. Umpleby, and R. H. Jones, "Partitioning glucose distribution/transport, disposal, and endogenous production during ivgtt," American Journal of Physiology-Endocrinology and Metabolism, vol. 282, no. 5, pp. E992-E1007, 2002. 
[41] C. Cobelli, G. Pacini, G. Toffolo, and L. Sacca, "Estimation of insulin sensitivity and glucose clearance from minimal model: new insights from labeled ivgtt," American Journal of Physiology-Endocrinology And Metabolism, vol. 250, no. 5, pp. E591-E598, 1986.

[42] J. Tiran, L. Avruch, and A. Albisser, "A circulation and organs model for insulin dynamics," American Journal of Physiology-Gastrointestinal and Liver Physiology, vol. 237, no. 4, pp. G331-G339, 1979.

[43] R. Srinivasan, A. H. Kadish, and R. Sridhar, "A mathematical model for the control mechanism of free fatty acid-glucose metabolism in normal humans," Computers and Biomedical Research, vol. 3, no. 2, pp. 146-165, 1970.

[44] C. Cobelli, G. Nucci, and S. Del Prato, "A physiological simulation model of the glucose-insulin system," in [Engineering in Medicine and Biology, 1999. 21st Annual Conference and the 1999 Annual Fall Meetring of the Biomedical Engineering Society] BMES/EMBS Conference, 1999. Proceedings of the First Joint, vol. 2. IEEE, 1999, pp. 999-vol.

[45] J. T. Sorensen, "A physiologic model of glucose metabolism in man and its use to design and assess improved insulin therapies for diabetes," Ph.D. dissertation, Massachusetts Institute of Technology, 1985.

[46] G. D. Mitsis and V. Z. Marmarelis, "Nonlinear modeling of glucose metabolism: comparison of parametric vs. nonparametric methods," in Engineering in Medicine and Biology Society, 200\%. EMBS 200\%. 29th Annual International Conference of the IEEE. IEEE, 2007, pp. 5967-5970.

[47] Z. Trajanoski, W. Regittnig, and P. Wach, "Simulation studies on neural predictive control of glucose using the subcutaneous route," Computer methods and programs in biomedicine, vol. 56, no. 2, pp. 133-139, 1998.

[48] J. Florian and R. S. Parker, "Empirical modeling for glucose control in critical care and diabetes," European journal of control, vol. 11, no. 6, pp. 601-616, 2005.

[49] R. Bellazzi, L. Ironi, R. Guglielmann, and M. Stefanelli, "Qualitative models and fuzzy systems: an integrated approach for learning from data," Artificial Intelligence in Medicine, vol. 14, no. 1, pp. 5-28, 1998.

[50] G. Bleckert, U. G. Oppel, and E. Salzsieder, "Mixed graphical models for simultaneous model identification and control applied to glucose-insulin metabolism," 
Computer methods and programs in biomedicine, vol. 56, no. 2, pp. 141-155, 1998.

[51] G. Segre, G. Turco, and G. Vercellone, "Modeling blood glucose and insulin kinetics in normal, diabetic and obese subjects," Diabetes, vol. 22, no. 2, pp. 94-103, 1973.

[52] R. N. Bergman, Y. Z. Ider, C. R. Bowden, and C. Cobelli, "Quantitative estimation of insulin sensitivity." American Journal of Physiology-Endocrinology And Metabolism, vol. 236, no. 6, p. E667, 1979.

[53] R. Bergman, "The minimal model: yesterday, today and tomorrow," The Minimal Model Approach and Determinants of Glucose Tolerance. RN Bergman and JC Lovejoy, ed. Louisiana State Univ. Press, Baton Rouge, pp. 3-50, 1997.

[54] M. J. Quon, C. Cochran, S. I. Taylor, and R. C. Eastman, "Non-insulinmediated glucose disappearance in subjects with iddm: discordance between experimental results and minimal model analysis," Diabetes, vol. 43, no. 7, pp. 890-896, 1994.

[55] K. M. Weber, I. Martin, J. D. Best, F. P. Alford, and R. C. Boston, "Alternative method for minimal model analysis of intravenous glucose tolerance data," American Journal of Physiology-Endocrinology and Metabolism, vol. 256, no. 4, pp. E524-E535, 1989.

[56] C. Cobelli and A. Ruggeri, "Evaluation of portal/peripheral route and of algorithms for insulin delivery in the closed-loop control of glucose in diabetes-a modeling study," Biomedical Engineering, IEEE Transactions on, no. 2, pp. 93-103, 1983.

[57] G. Pacini and C. Cobelli, "Estimation of $\beta$-cell secretion and insulin hepatic extraction by the minimal modelling technique," Computer methods and programs in biomedicine, vol. 32, no. 3, pp. 241-248, 1990.

[58] M. Berger and D. Rodbard, "Computer simulation of plasma insulin and glucose dynamics after subcutaneous insulin injection," Diabetes care, vol. 12, no. 10, pp. 725-736, 1989.

[59] E. Salzsieder, G. Albrecht, U. Fischer, and E.-J. Freyse, "Kinetic modeling of the glucoregulatory system to improve insulin therapy," Biomedical Engineering, IEEE Transactions on, no. 10, pp. 846-855, 1985. 
[60] U. Fischer, W. Schenk, E. Salzsieder, G. Albrecht, P. Abel, and E.-J. Freyse, "Does physiological blood glucose control require an adaptive control strategy?" Biomedical Engineering, IEEE Transactions on, no. 8, pp. 575-582, 1987.

[61] U. Fischer, E. Salzsieder, E.-J. Freyse, and G. Albrecht, "Experimental validation of a glucose-insulin control model to simulate patterns in glucose turnover," Computer methods and programs in biomedicine, vol. 32, no. 3, pp. 249-258, 1990.

[62] M. Boroujerdi, A. Umpleby, R. Jones, and P. Sonksen, "A simulation model for glucose kinetics and estimates of glucose utilization rate in type 1 diabetic patients," American Journal of Physiology-Endocrinology and Metabolism, vol. 268, no. 4, pp. E766-E774, 1995.

[63] E. Lehmann and T. Deutsch, "A physiological model of glucose-insulin interaction in type 1 diabetes mellitus," Journal of biomedical engineering, vol. 14, no. 3, pp. 235-242, 1992.

[64] E. Lehmann, "Preliminary experience with the internet release of aidaan interactive educational diabetes simulator," Computer methods and programs in biomedicine, vol. 56, no. 2, pp. 109-132, 1998.

[65] G. Steil, B. Clark, S. Kanderian, and K. Rebrin, "Modeling insulin action for development of a closed-loop artificial pancreas," Diabetes technology \& therapeutics, vol. 7, no. 1, pp. 94-108, 2005.

[66] I. M. Tolić, E. Mosekilde, and J. Sturis, "Modeling the insulin-glucose feedback system: the significance of pulsatile insulin secretion," Journal of theoretical biology, vol. 207, no. 3, pp. 361-375, 2000.

[67] P. Vicini and C. Cobelli, "The iterative two-stage population approach to ivgtt minimal modeling: improved precision with reduced sampling," American Journal of Physiology-Endocrinology and Metabolism, vol. 280, no. 1, pp. E179-E186, 2001.

[68] K. E. Andersen and M. Højbjerre, "A population-based bayesian approach to the minimal model of glucose and insulin homeostasis," Statistics in medicine, vol. 24, no. 15, pp. 2381-2400, 2005.

[69] K. Engelborghs, V. Lemaire, J. Bélair, and D. Roose, "Numerical bifurcation analysis of delay differential equations arising from physiological modeling," Journal of mathematical biology, vol. 42, no. 4, pp. 361-385, 2001. 
[70] E. Kraegen and D. Chisholm, "Insulin responses to varying profiles of subcutaneous insulin infusion: kinetic modelling studies," Diabetologia, vol. 26, no. 3, pp. 208-213, 1984.

[71] W. R. Puckett and E. N. Lightfoot, "A model for multiple subcutaneous insulin injections developed from individual diabetic patient data," American Journal of Physiology-Endocrinology and Metabolism, vol. 269, no. 6, pp. E1115-E1124, 1995.

[72] P. Wach, Z. Trajanoski, P. Kotanko, and F. Skrabal, "Numerical approximation of mathematical model for absorption of subcutaneously injected insulin," Medical and Biological Engineering and Computing, vol. 33, no. 1, pp. 18-23, 1995.

[73] J. Tiran, K. R. Galle, and D. Porte Jr, "A simulation model of extracellular glucose distribution in the human body," Annals of biomedical engineering, vol. 3, no. 1, pp. 34-46, 1975.

[74] J. R. Guyton, R. O. Foster, J. S. Soeldner, M. H. Tan, C. B. Kahn, L. Koncz, and R. E. Gleason, "A model of glucose-insulin homeostasis in man that incorporates the heterogeneous fast pool theory of pancreatic insulin release," Diabetes, vol. 27, no. 10, pp. 1027-1042, 1978.

[75] J. Kim, G. M. Saidel, and M. E. Cabrera, "Multi-scale computational model of fuel homeostasis during exercise: effect of hormonal control," Annals of biomedical engineering, vol. 35, no. 1, pp. 69-90, 2007.

[76] C. D. Man, R. A. Rizza, and C. Cobelli, "Meal simulation model of the glucoseinsulin system," Biomedical Engineering, IEEE Transactions on, vol. 54, no. 10, pp. 1740-1749, 2007.

[77] M. Breton, C. Cobelli, M. Dalla, and B. Kovatchev, "Method, system and computer simulation environment for testing of monitoring and control strategies in diabetes," Patent, Jul. 7, 2010, eP Patent App. EP20,080,780,896.

[78] R. Basu, B. Di Camillo, G. Toffolo, A. Basu, P. Shah, A. Vella, R. Rizza, and C. Cobelli, "Use of a novel triple-tracer approach to assess postprandial glucose metabolism," American Journal of Physiology-Endocrinology And Metabolism, vol. 284, no. 1, pp. E55-E69, 2003.

[79] D. C. Klonoff, "The artificial pancreas: how sweet engineering will solve bitter problems," Journal of diabetes science and technology, vol. 1, no. 1, pp. 72-81, 2007. 
[80] A. H. Kadish, "Automation control of blood sugar a servomechanism for glucose monitoring and control." ASAIO Journal, vol. 9, no. 1, pp. 363-367, 1963.

[81] A. Albisser, B. Leibel, T. Ewart, Z. Davidovac, C. Botz, and W. Zingg, "An artificial endocrine pancreas," Diabetes, vol. 23, no. 5, pp. 389-396, 1974.

[82] A. M. Albisser, B. Leibel, T. Ewart, Z. Davidovac, C. Botz, W. Zingg, H. Schipper, and R. Gander, "Clinical control of diabetes by the artificial pancreas," Diabetes, vol. 23, no. 5, pp. 397-404, 1974.

[83] A. H. Clemens, P. H. Chang, and R. W. Myers, "The development of Biostator, a Glucose Controlled Insulin Infusion System (GCIIS)," Horm. Metab. Res., vol. Suppl 7, pp. 23-33, 1977.

[84] C. K. Botz, "An improved control algorithm for an artificial beta-cell," IEEE Trans Biomed Eng, vol. 23, no. 3, pp. 252-255, May 1976.

[85] E. Kraegen, L. Campbell, Y. Chia, H. Meier, and L. Lazarus, "Control of blood glucose in diabetics using an artificial pancreas*," Australian and New Zealand journal of medicine, vol. 7, no. 3, pp. 280-286, 1977.

[86] E. B. Marliss, F. T. Murray, E. F. Stokes, B. Zinman, A. F. Nakhooda, A. Denoga, B. S. Leibel, and A. M. Albisser, "Normalization of glycemia in diabetics during meals with insulin and glucagon delivery by the artificial pancreas," Diabetes, vol. 26, no. 7, pp. 663-672, 1977.

[87] H. M. Broekhuyse, H. D. Nelson, B. Zinman, and A. Albisser, "Comparison of algorithms for the closed-loop control of blood glucose using the artificial beta cell," Biomedical Engineering, IEEE Transactions on, no. 10, pp. 678-687, 1981.

[88] S. A. Weinzimer, G. M. Steil, K. L. Swan, J. Dziura, N. Kurtz, and W. V. Tamborlane, "Fully automated closed-loop insulin delivery versus semiautomated hybrid control in pediatric patients with type 1 diabetes using an artificial pancreas," Diabetes care, vol. 31, no. 5, pp. 934-939, 2008.

[89] G. M. Grodsky, "A threshold distribution hypothesis for packet storage of insulin and its mathematical modeling," Journal of Clinical Investigation, vol. 51, no. 8, p. 2047, 1972.

[90] A. D. Cherrington, D. Sindelar, D. Edgerton, K. Steiner, and O. P. McGuinness, "Physiological consequences of phasic insulin release in the normal animal," Diabetes, vol. 51, no. suppl 1, pp. S103-S108, 2002. 
[91] G. M. Steil, K. Rebrin, R. Janowski, C. Darwin, and M. F. Saad, "Modeling $\beta$ cell insulin secretion-implications for closed-loop glucose homeostasis," Diabetes technology \&f therapeutics, vol. 5, no. 6, pp. 953-964, 2003.

[92] G. Swan, "An optimal control model of diabetes mellitus," Bulletin of mathematical biology, vol. 44, no. 6, pp. 793-808, 1982.

[93] R. Ollerton, "Application of optimal control theory to diabetes mellitus," International Journal of Control, vol. 50, no. 6, pp. 2503-2522, 1989.

[94] M. E. Fisher and K. L. Teo, "Optimal insulin infusion resulting from a mathematical model of blood glucose dynamics," Biomedical Engineering, IEEE Transactions on, vol. 36, no. 4, pp. 479-486, 1989.

[95] L. Magni, D. M. Raimondo, L. Bossi, C. Dalla Man, G. De Nicolao, B. Kovatchev, and C. Cobelli, "Model predictive control of type 1 diabetes: an in silico trial," Journal of diabetes science and technology, vol. 1, no. 6, pp. 804$812,2007$.

[96] M. Morari and N. L. Ricker, "Model predictive control toolbox," The MathWorks, Inc., Natick, MA, 1994.

[97] S. M. Lynch and B. W. Bequette, "Model predictive control of blood glucose in type i diabetics using subcutaneous glucose measurements," in Proceedings of the American Control Conference, 2002, pp. 4039-4043.

[98] M. E. Fisher, "A semiclosed-loop algorithm for the control of blood glucose levels in diabetics," Biomedical Engineering, IEEE Transactions on, vol. 38, no. 1, pp. 57-61, 1991.

[99] R. Hovorka, V. Canonico, L. J. Chassin, U. Haueter, M. Massi-Benedetti, M. O. Federici, T. R. Pieber, H. C. Schaller, L. Schaupp, T. Vering et al., "Nonlinear model predictive control of glucose concentration in subjects with type 1 diabetes," Physiological measurement, vol. 25, no. 4, p. 905, 2004.

[100] C. J. Tomlin and J. D. Axelrod, "Biology by numbers: mathematical modelling in developmental biology," Nature Reviews Genetics, vol. 8, no. 5, pp. 331-340, 2007.

[101] G. T. Reeves and S. E. Fraser, "Biological systems from an engineer's point of view," PLoS biology, vol. 7, no. 1, p. e1000021, 2009.

[102] D. Group, "The artificial pancreas for people with type 1 diabetes," 2011. 
[103] R. Hovorka, V. Canonico, L. J. Chassin, U. Haueter, M. Massi-Benedetti, M. O. Federici, T. R. Pieber, H. C. Schaller, L. Schaupp, T. Vering et al., "Nonlinear model predictive control of glucose concentration in subjects with type 1 diabetes," Physiological measurement, vol. 25, no. 4, p. 905, 2004.

[104] D. Boiroux, "Nonlinear model predictive control for an artificial pancreas," Master's thesis, Technical University of Denmark, DTU, DK-2800 Kgs. Lyngby, Denmark, 2009.

[105] M. Suzanne and J. R. Wands, "Alzheimer's disease is type 3 diabetesevidence reviewed," Journal of diabetes science and technology, vol. 2, no. 6, pp. 11011113, 2008.

[106] S. Wild, G. Roglic, A. Green, R. Sicree, and H. King, "Global prevalence of diabetes: Estimates for the year 2000 and projections for 2030," Diabetes Care, vol. 27, no. 5, pp. 1047-1053, 2004.

[107] S. N. Mehta and J. I. Wolfsdorf, "Contemporary management of patients with type 1 diabetes," Endocrinology and Metabolism Clinics of North America, vol. 39, no. 3, pp. $573-593,2010$.

[108] "Diagnosis and classification of diabetes mellitus," Diabetes Care, vol. 27, no. suppl 1, pp. s5-s10, 2004.

[109] S. Ahmad, Diabetes: An Old Disease, a New Insight, ser. Advances in Experimental Medicine and Biology Series. Springer Science+Business Media, 2012.

[110] "Diabetes mellitus," in Encyclopedia of Child Behavior and Development, S. Goldstein and J. Naglieri, Eds. Springer US, 2011, pp. 501-501.

[111] World Health Organization and International Diabetes Federation, "Definition and Diagnosis of diabetes Mellitus and Intermediate Hyperglycemia," 2006.

[112] The Global Diabetes Community. (2013, May) Diabetes types.

[113] The Canadian Diabetes Association Clinical Practice Guideline. (2013, May) Type 1 diabetes: the basics.

[114] Mayo Clinic Staff. (2013, May) Gestational diabetes.

[115] L. M. S. de Souza Rugolo, M. R. Bentlin, and C. E. P. Trindade, "Preeclampsia: Effect on the fetus and newborn," NeoReviews, vol. 12, no. 4, pp. e198-e206, 2011. 
[116] J. Carrier, Managing long-term conditions and chronic illness in primary care: a guide to good practice. Abingdon New York: Routledge, 2009.

[117] W. Herman, The evidence base for diabetes care. Chichester, West Sussex, UK Hoboken, NJ: Wiley-Blackwell, 2010.

[118] H. Shamoon, H. Duffy, N. Fleischer, S. Engel, P. Saenger, M. Strelzyn, M. Litwak, J. Wylierosett, A. Farkash, D. Geiger et al., "The effect of intensive treatment of diabetes on the development and progression of long-term complications in insulin-dependent diabetes-mellitus," New England Journal of Medicine, vol. 329, no. 14, pp. 977-986, 1993.

[119] UK Prospective Diabetes Study (UKPDS) Group, "Intensive blood-glucose control with sulphonylureas or insulin compared with conventional treatment and risk of complications in patients with type 2 diabetes (ukpds 33)."

[120] Mayo Clinic Staff, "Diabetes: Treatments and drugs," Online, July 2014.

[121] T. Neithercott, "Continuous glucose monitors 2014," Online, Jan 2014.

[122] The New York Times, "Diabetes, type 2 in-depth report," Online, June 2013.

[123] R. Hillson, Diabetes : the complete guide : the essential introduction to managing diabetes. London: Vermilion, 2002.

[124] S. I. J. M. LeRoith, Derek; Taylor, Diabetes Mellitus: A Fundamental and Clinical Text. Lippincott Williams \& Wilkins, 2004.

[125] American Diabetes Association, "Insulin basics," Online, Jul 2014.

[126] Joslin Diabetes Center, "Insulin injections vs. insulin pump," Online, Feb 2015. 


\section{Appendix A}

\section{Diabetes Mellitus}

The chronic metabolic disorder known as Diabetes Mellitus (DM) is one the fastest growing disorders in the world. Like most diseases, DM has a big impact on the patient's economic, health and social aspect of life. The World Health Organization (WHO) estimated that there are currently 171 million people around the world that suffer from diabetes in the year 2000. However, this number is expected to increase to approximately 366 million in the year 2030. In addition to this, WHO suggests that this chronic metabolic disorder is one of the world's costly diseases [106].

This section introduces the metabolic disorder and the three most common types of DM. It then goes on to discuss some of the complications a person with this disorder might face. Lastly, it investigates various treatment alternatives that are currently available, and some emerging therapies.

Diabetes Mellitus, commonly known as diabetes, is a disease that is characterized by the inadequate regulation of glucose concentrations (glycemia) within the human body. WHO defines diabetes as the absolute or relative deficit of insulin which in turn increases a person's blood sugar level (specifically in the venous blood) over 7.0 $\mathrm{mmol} / \mathrm{L}(126 \mathrm{mg} / \mathrm{dL})$ after an fasting overnight (i.e. not consuming anything after midnight) or over $11.1 \mathrm{mmol} / \mathrm{L}(200 \mathrm{mg} / \mathrm{dL})$ after two hours of consuming a meal that 
is rich in carbohydrates, or after two hours after an oral tolerance test. Note that the Oral Glucose Tolerance Test (OGTT) is the measure of the plasma glucose levels both before and after two hours after glucose is given orally to the patient. [107,108].

\section{A.1 Regulation of blood glucose}

In order to regulate the glucose levels in the blood stream, a negative feedback loop is required. This negative feedback loop acts in conjunction with the hormones that are present in the body called insulin and glucagon. When the level of glucose in the blood stream is high, the $\beta$-cells of the islet of langerhans in the pancreas secrete insulin. The secreted insulin then sends a message to the liver, to start converting all the excess glucose to glycogen and store it. In addition, the presence of insulin in the blood stream triggers the muscle and adipose tissue to absorb more glucose; and thus in turn achieving homeostasis [109].

Similarly, when the glucose concentration in the blood is low, the $\alpha$-cells of the pancreas simulates the release of the hormone glucagon. Glucagon sends a message to the liver to start converting the stored glycogen into glucose, and, release it into the blood stream. This in turn enables the glucose concentration to return to its normal level.

Patients who are inflicted with diabetes could have problems with any one of these processes. This includes, but is not limited to:

- The person's body has problem with the synthesis or secretion of the hormone, insulin;

- The person's body has developed a resistance to the hormone insulin;

- The person's body has a subnormal production of the hormone insulin $[109,110]$. 
Note that in a recent study conducted by researchers at the Rhode Island Hospital and the Brown Medical School it was uncovered that insulin and its related proteins are also produced brain [105]. This thesis does not take into consideration insulin that is produced by the brain since it is predominantly dealing with Type 3 diabetes (see section A.2).

\section{A.2 Classification of Diabetes Mellitus}

According to WHO, diabetes can be classified by three major types:

1. Type 1;

2. Type 2; or,

3. Gestational diabetes.

In addition to these three types there are also other secondary types of diabetes which are far less common than the three previously mentioned[110,111]. These include, but are not limited to: Maturity Onset Diabetes of the Young, steroid induced diabetes, Brittle diabetes etc. [112].

\section{A.2.1 Type 1 Diabetes Mellitus}

Type 1 diabetes mellitus (T1DM), is also known as the insulin depended diabetes mellitus (IDDM), or juvenile diabetes mellitus as it affects mostly younger people. This particular type of autoimmune disease occurs when the patient's $\beta$-cells located in their pancreas stops producing the hormone insulin. This in turn causes the mass of the $\beta$-cells to drastically reduce as the cells die. This entire process takes years before any clinical symptoms appear [109]. 
However, the mechanism and reasoning behind which this appears, is poorly understood by the scientific community. Nevertheless, some genetic studies have claimed that the HLA (human leukocyte antigen) gene, that is located on chromosome 6 is closely related to T1DM. In addition to this gene, there also needs to be some environmental factors that trigger this autoimmune reaction to destroy the pancreatic $\beta$-cells. However, this environmental is not yet thoroughly studied [113].

\section{A.2.2 Type 2 Diabetes Mellitus}

Type 2 diabetes mellitus (T2DM), is known as the non-insulin depended diabetes mellitus (NIDDM). This type of diabetes is inflicted upon a patient whose $\beta$-cells in their pancreas does not produce enough insulin. Or, the cells in the entire body do not accurately utilize the insulin that is produced. Furthermore, it should be noted that this particular type of diabetes accounts for approximately $90 \%$ of diabetics worldwide [109, 111].

T2DM can be further divided into two subcategories - diabetes with obesity and diabetes without obesity. Research shows that the patients who are inflicted with diabetes and obesity have altered their cell receptor due to the increase in abdominal fat and thus developed a resistance to endogenous insulin. However, patients with diabetes, who are not obese have only a little resistance at the cell receptor level, but have a substantial deficiency in the production and release of insulin. Thus, there is a direct correlation between obesity and diabetes, many patients can be simply 'cured' by changing the patient's diet and lifestyle [109].

\section{A.2.3 Gestational Diabetes Mellitus}

As its name suggests, Gestational Diabetes Mellitus (GDM) occurs when a woman is pregnant. This occurs because the woman's body cannot handle the effects of 
a growing baby and the resultant hormone changes. Almost all women have some degree of intolerance to glucose during pregnancy which results in high glucose levels. However, $96 \%$ of women do not have a high enough glucose level to consider it to be diabetes. The $4 \%$ of women who are diagnosed are usually done so during the third trimester. This is because as the baby gets bigger there is a greater amount of glucose fluctuation and accelerated starvation that the woman's body experiences. In addition, by the end of the first trimester there is an increase in insulin secretion by the placenta and a decrease in insulin sensitivity. This results in a woman's body developing insulin resistance which thus causes diabetes [109].

It should be noted that, unlike other forms of diabetes which are life long, a patient inflicted with GDM generally returns to their normal glucose regulation once the baby is born. But, once inflicted with GDM, the chances of being diagnosed with T2DM increases significantly [114]. Additionally, there are a number of complications that may develop including: increased risk of mortality for both the mother and the baby, pre-eclampsia, intrauterine growth retardation, premature delivery and still birth [115].

\section{A.3 Long Term Effects}

There are some people throughout the world that live with DM without encountering any serious side effects from the disease. This is because if one manages their blood glucose levels vigorously, the body does not react differently to itself managing the glucose levels or an external control managing the blood glucose levels. The effects of diabetes are not consistent and each person's body would react differently. It is not the aim of this document to outline the details about the disease, thus only an overview of the complications resulting from this disease will be outlined. 
As noted earlier on in this chapter, DM is characterised by either too high or too low blood glucose concentrations. Blood as we know travels throughout the body and hence the complications that could result from this are not restricted to a single part of the body. Increased blood sugar levels could eventually damage blood vessels, nerves, and the organs in the human body. This is mainly due to the fact that high concentrations of blood glucose damages the blood vessels and low blood glucose concentrations starves the rest of the body from getting one of the main nutrients sugar. Some of the main complications include but are not limited to: Cardiovascular disease; Hypoglycemia; Nephropathy; Neuropathy; and, Retinopathy $[116,117]$.

Cardiovascular diseases pertain to any disease relating to the cardiovascular system of the human body and can range from diseases relating to blood vessels to strokes and heart attacks. This group of diseases is the leading cause of death amongst diabetic patients with coronary heart disease and hypertension at the top of this chart. Coronary heart disease is caused by fatty deposits in the arteries that feed the heart (high cholesterol). Hypertension on the other hand is most commonly known as high blood pressure $[116,117]$.

Hypoglycemia, meaning low blood sugar, is just as dangerous as high blood sugar. This is most often triggered by one of the following conditions:

- not eating often enough, or, eating too little, which leads your body to think you are starving;

- too much physical activity without eating enough, this also deceives your body from thinking it is starving since the amount of energy you are expending is not nearly enough to replenish it; or,

- too much insulin. 
This complication is easy to treat provided the person undergoing it knows that their blood sugar is low (people who are diabetic are often informed of the effects of low blood sugar such as headaches, fatigue, etc.). The problem with this complication is that the more often one's body gets used to being in this state the more likely it is going to think it is normal. This can cause devastating effects such as unconsciousness or even death $[116,117]$.

Nephropathy (i.e. kidney disease) is also a common complication of diabetes. Unregulated glucose concentrations in the human body can eventually lead to the slow deterioration of one's kidneys and in turn its function. This eventually could result in kidney failure (i.e. end-stage renal disease (ESRD)) or other renal diseases. The way this occurs is because the kidneys are known to filter waste from the blood stream. However, damage to blood cells due to high glucose concentrations could tip the balance of this delicate filtering system which would then lead into the fore mentioned diseases $[116,117]$.

Neuropathy is complications relating to nerve damage. This is caused by high glucose concentrations that damage the capillaries (smallest blood vessels in the human body) that provide nutrients to various parts of the body. This particular group of problems can also range from a small problem that can be overcome to a major disability or even death. This usually starts with burning, numbness and/or tingling at the tips of your extremities (i.e. fingers and toes). This usually then continues upwards throughout the body which eventually could damage an entire limb or organ. The damage of vital organs such as the heart or brain could then result in death. Nerve damage could also be part of the autonomic nervous system. As its name suggests this system functions automatically, such as breathing, sexual functions, digestion, regulation of body temperature, etc. damage to these key automatic functions of the body could have devastating effects and could eventually lead to death $[116,117]$. 
The last major section this document will cover is retinopathy (diseases related to the eye). This group of diseases is often progressive. It starts off with slowly destroying small blood vessels in the retina causing vision loss and eventually leading to blindness (i.e. proliferative retinopathy). Diabetic patients also have an increased risk of other serious vision complication such as glaucoma and cataracts $[116,117]$.

\section{A.4 Current techniques available to monitor and treat diabetes}

As mentioned before, although there is no cure for DM, clinical trials time and time again have proven how proper glucose management could reduce and possible eliminate the risks posed by diabetes $[118,119]$. The Diabetes Control and Complications Trial (DCCT) was the first clinical trial to prove the tremendous benefit to diabetic patients if they rigorously maintain their blood glucose levels. And, although this was targeted particularly at T1DM, it is hypothesised that this could easily be extended to T2DM. Not long after that, in 1998, the UK Prospective Diabetes Study (UKPDS) Group conducted a study that verified the same improvements can be seen in patients with T2DM provided the same rigorous monitoring of blood glucose levels in maintained.

There are a number of ways to monitor and treat the disease depending on the variation of DM one is inflicted with. Before attempting to treat the disease one must first diagnose it and monitor it. There are mainly four blood tests that allow a medical doctor to diagnose a patient - an A1C (also known as Glycated hemoglobin (A1C), hemoglobin A1c, HbA1c, or glycohemoglobin) test; a fasting plasma glucose (FPG) test; an Oral Glucose Tolerance Test (OGTT); a random blood glucose test. The first blood test is extremely common as it indicates one's average blood sugar 
Table A1: Significance of the results of blood tests used to diagnose diabetes mellitus [35].

\begin{tabular}{|c|c|c|}
\hline Blood Test used to Diagnose Diabetes & Result & Diagnosis \\
\hline \multirow{3}{*}{ Glycated hemoglobin (A1C) test } & $\geq 6.5 \%$ & Diabetic \\
\hline & $5.7 \%-6.4 \%$ & Pre-diabetic \\
\hline & $\leq 5.7 \%$ & Normal \\
\hline \multirow{3}{*}{ Fasting Plasma Glucose test } & $\geq 126 \mathrm{mg} / \mathrm{dL}(7 \mathrm{mmol} / \mathrm{L})$ & Diabetic \\
\hline & $100 \mathrm{mg} / \mathrm{dL}-125 \mathrm{mg} / \mathrm{dL}(5.6 \mathrm{mmol} / \mathrm{L}-6.9 \mathrm{mmol} / \mathrm{L})$ & Pre-diabetic \\
\hline & $\leq 100 \mathrm{mg} / \mathrm{dL}(5.6 \mathrm{mmol} / \mathrm{L})$ & Normal \\
\hline \multirow{3}{*}{ Oral glucose tolerance test (OGTT)) } & $\geq 200 \mathrm{mg} / \mathrm{dL}(11.1 \mathrm{mmol} / \mathrm{L})$ & Diabetic \\
\hline & $140 \mathrm{mg} / \mathrm{dL}-199 \mathrm{mg} / \mathrm{dL}(7.8 \mathrm{mmol} / \mathrm{L}-11.0 \mathrm{mmol} / \mathrm{L})$ & Pre-diabetic \\
\hline & $\leq 140 \mathrm{mg} / \mathrm{dL}(7.8 \mathrm{mmol} / \mathrm{L})$ & Normal \\
\hline \multirow{3}{*}{ Random blood sugar test } & $\geq 200 \mathrm{mg} / \mathrm{dL}(11.1 \mathrm{mmol} / \mathrm{L})$ & Diabetic \\
\hline & $\mathrm{N} / \mathrm{A}$ & Pre-diabetic \\
\hline & $\mathrm{N} / \mathrm{A}$ & Normal \\
\hline
\end{tabular}


for the past two to three months. The significance of this test results can be seen in Table A1. FPG on the other hand requires a blood sample to be taken after the patient has fasted overnight. OGTT can be thought of as a modified FPG test. Just like the FPG test the patient is required to fast overnight. Their blood glucose level is then measured and recorded. A sugary liquid is then ingested by the patient and their blood glucose level is then measured periodically over the course of the next two hours. Lastly, as it's name suggests the random blood sugar test requires a patient's blood sugar to be tested at any given time regardless of when the patient ate or exercised last. It should be noted that all the above mentioned tests are performed at least twice before a patient is officially diagnosed [120].

\section{A.4.1 Monitoring glucose levels}

Upon diagnosis from a medical doctor patients are often required to monitor their blood glucose levels. This is usually done with a glucose sensor. Currently there are many types of glucose sensors that are available in the market. Sensors range from the basic to more advanced devices that could store measured glucose levels to review with a doctor at a later time. Additionally, some sensors also come with an application that can be downloaded on to your computer, tablet or phone. This gives the patient the ability save the data so that he/she can review the data at a later time. Currently there are basically two types of glucose monitors used widely: the first the Continuous Glucose Monitoring (CGM) system; and, the second, but more common sensor, the finger-prick test $[121,122]$.

The finger-prick test includes three devices: one used to prick the patient's finger (or any part of the body) such that a drop of blood can be easily drawn. The second includes a chemically treated strip that is attached to the third device which is the glucose meter itself. The test involves pin pricking the patient's body (usually 
finger) to release a drop of blood that is then 'sucked' into the chemically treated test strip that the already inserted into the glucose monitor. Within a couple of seconds the glucose monitor is able to read the blood sugar level of the drop of blood in the chemically treated strip. This methodology is preferred amongst most patients because it easily hidden and does not pose a stigma [121-123].

As its name suggests the CGM continuously monitors the blood glucose levels of the patient and alerts the patient when their blood glucose is too high or too low. This device is invasive and is continually attached to the patient's body. Note that this system is often recommended to patients who have huge fluctuations in their blood glucose levels. These monitors are also often used on children and elderly patients. When the monitor was first introduced, its bulky design turned away many patients from actually using it. However, technology has advanced and more real time continuous glucose monitors are available that are sleek [121,122].

\section{A.4.2 Treatment}

For patients who are inflicted with DM, once their blood glucose levels are measured they may need to ingest something to adjust their blood sugar accordingly. Depending on the type of diabetes the patient is diagnosed with, different 'treatment' options are prescribed. These include Medical Nutrition Therapy (MNT); exercise; pills; and/or insulin. MNT and exercise is the foundation to any diabetic treatment, it includes educating the patient as to how to maintain a healthy diet; and use exercise as a way to lower blood sugar by forcing their body to convert the excess sugar into energy. Pills (i.e oral or other medication) is the second stage of treating T2DM. Generally the first medication that is prescribed is known as Metformin. This particular drug aims to decrease the glucose production in the liver and the amount of glucose absorbed from food that is ingested by the patient [124]. Some other medication include stimulating 
the patient's pancreas to produce and release more insulin [120].

Table A2: Major insulin types and their effective times [40].

\begin{tabular}{|c|c|c|c|c|}
\hline $\begin{array}{l}\text { Types of } \\
\text { Insulin }\end{array}$ & $\begin{array}{l}\text { Time to reach } \\
\text { bloodstream } \\
\text { after injection }\end{array}$ & $\begin{array}{c}\text { Peak } \\
\text { time after } \\
\text { injection }\end{array}$ & $\begin{array}{l}\text { Effective } \\
\text { time after } \\
\text { injection }\end{array}$ & Example \\
\hline $\begin{array}{l}\text { Rapid-acting } \\
\text { insulin }\end{array}$ & 15 minutes & 1 hour & $2-4$ hours & $\begin{array}{l}\text { - Insulin glulisine } \\
\text { (Apidra) } \\
\text { - Insulin lispro } \\
\text { (Humalog) } \\
\text { - Insulin aspart } \\
\text { (NovoLog) }\end{array}$ \\
\hline $\begin{array}{l}\text { Regular or } \\
\text { Short-acting } \\
\text { insulin }\end{array}$ & 30 minutes & $2-3$ hours & $3-6$ hours & $\begin{array}{l}\text { - Humulin R } \\
\text { - Novolin R }\end{array}$ \\
\hline $\begin{array}{l}\text { Intermediate- } \\
\text { acting insulin }\end{array}$ & $2-4$ hours & $4-12$ hours & $12-18$ hours & $\begin{array}{l}\text { NPH } \\
(\text { Humulin N, } \\
\text { Novolin N) }\end{array}$ \\
\hline $\begin{array}{l}\text { Long-acting } \\
\text { insulin }\end{array}$ & $\geq 4$ hours & $\begin{array}{c}\text { Fairly even } \\
\text { over } 24 \text { hours }\end{array}$ & $\leq 24$ hours & $\begin{array}{l}\text { - Insulin detemir } \\
\text { (Levemir) } \\
\text { - Insulin glargine } \\
\text { (Lantus) }\end{array}$ \\
\hline
\end{tabular}

Lastly, insulin therapy. This includes the patient injecting themselves with insulin. People who are inflicted with T1DM are prescribed insulin therapy in the early stages. However, people with T2DM are often prescribed insulin therapy when the body does not respond to oral medications and the patient does not exercise enough to lower blood glucose levels. The reason that insulin is separated from other oral medication is because insulin cannot be ingested like a pill this is because it would be broken down tremendously during digestion just like other proteins. Thus it must be injected, 
and it must be injected into the body under the fat so that it is injected directly into your blood stream. Depending on the diabetic type stage and therapy the patient is undergoing there are four major types of insulin: rapid-acting; regular or short-acting; intermediate-acting; and, long-acting. Details of their effectiveness are outlined in Table A2. Additionally, a mixture of these types of insulin could also be prescribed. These mixtures are available in pre-mixed packs or separately [125].

Like, the glucose sensor, the insulin is usually injected into the body by two major means: a manual insulin injection, or an insulin pump. Currently, insulin injections require far less education and training to the user. This is because the insulin pump needs to be closely monitored so that not too much insulin in injected into the body. Additionally, it is also a cheaper of the two options. On the other hand, the insulin pump delivers continuous doses of insulin to the human body, much like the pancreas. This prevents fewer peaks or troughs in blood glucose levels. Additionally, like the CGM, this device in continually injected into the patient's body offering the patient to prick themselves once in three days as opposed to 15-18 times in three days. Lastly, the biggest advantage of using the insulin pump is the ease and flexibility of adjusting insulin delivery level according to you current activity [126]. 